\title{
A Hipparcos study of the Hyades open cluster
}

\section{Improved colour-absolute magnitude and Hertzsprung-Russell diagrams}

\author{
J. H. J. de Bruijne, R. Hoogerwerf, and P. T. de Zeeuw
}

\begin{abstract}
Sterrewacht Leiden, Postbus 9513, 2300 RA Leiden, The Netherlands
Received 13 June 2000 / Accepted 24 November 2000

Abstract. Hipparcos parallaxes fix distances to individual stars in the Hyades cluster with an accuracy of $\sim 6$ percent. We use the Hipparcos proper motions, which have a larger relative precision than the trigonometric parallaxes, to derive $\sim 3$ times more precise distance estimates, by assuming that all members share the same space motion. An investigation of the available kinematic data confirms that the Hyades velocity field does not contain significant structure in the form of rotation and/or shear, but is fully consistent with a common space motion plus a (one-dimensional) internal velocity dispersion of $\sim 0.30 \mathrm{~km} \mathrm{~s}^{-1}$. The improved parallaxes as a set are statistically consistent with the Hipparcos parallaxes. The maximum expected systematic error in the proper motion-based parallaxes for stars in the outer regions of the cluster (i.e., beyond $\sim 2$ tidal radii $\sim 20$ pc) is $\lesssim 0.30$ mas. The new parallaxes confirm that the Hipparcos measurements are correlated on small angular scales, consistent with the limits specified in the Hipparcos Catalogue, though with significantly smaller "amplitudes" than claimed by Narayanan \& Gould. We use the Tycho-2 long time-baseline astrometric catalogue to derive a set of independent proper motion-based parallaxes for the Hipparcos members. The new parallaxes provide a uniquely sharp view of the three-dimensional structure of the Hyades. The colour-absolute magnitude diagram of the cluster based on the new parallaxes shows a well-defined main sequence with two "gaps" / turn-offs". These features provide the first direct observational support of Böhm-Vitense's prediction that (the onset of) surface convection in stars significantly affects their $(B-V)$ colours. We present and discuss the theoretical Hertzsprung-Russell diagram ( $\log L$ versus $\log T_{\text {eff }}$ ) for an objectively defined set of 88 high-fidelity members of the cluster as well as the $\delta$ Scuti star $\theta^{2}$ Tau, the giants $\delta^{1}, \theta^{1}, \epsilon$, and $\gamma$ Tau, and the white dwarfs V471 Tau and HD 27483 (all of which are also members). The precision with which the new parallaxes place individual Hyades in the Hertzsprung-Russell diagram is limited by (systematic) uncertainties related to the transformations from observed colours and absolute magnitudes to effective temperatures and luminosities. The new parallaxes provide stringent constraints on the calibration of such transformations when combined with detailed theoretical stellar evolutionary modelling, tailored to the chemical composition and age of the Hyades, over the large stellar mass range of the cluster probed by Hipparcos.
\end{abstract}

Key words. astrometry - stars: distances - stars: fundamental parameters - stars: Hertzsprung-Russell diagram - Galaxy open clusters and associations: individual: Hyades

\section{Introduction}

The Hyades open cluster has for most of the past century been an important calibrator of many astrophysical relations, e.g., the absolute magnitude-spectral type and the mass-luminosity relation. The cluster has been the subject of numerous investigations (e.g., van Bueren 1952; Pels et al. 1975; Reid 1993; Perryman et al. 1998) addressing, e.g., cluster dynamics and evolution, the distance scale in the Universe (e.g., Hodge \& Wallerstein 1966; van den Bergh 1977), and the calibration of spectroscopic radial velocities (e.g., Petrie 1963; Dravins et al. 1999).

The significance of the Hyades is nowadays mainly limited to the broad field of stellar structure and evolution.

Send offprint requests to: P. T. de Zeeuw, e-mail: tim@strw.leidenuniv.nl
Open clusters in general form an ideal laboratory to study star formation, structure, and evolution theories, as their members are thought to have been formed simultaneously from the same molecular cloud material. As a result, they have (1) the same age, at least to within a few Myr, (2) the same distance, neglecting the intrinsic size which is at maximum 10-20 pc, (3) the same initial element abundances, and (4) the same space motion, neglecting the internal velocity dispersion which is similar to the velocity dispersion within the parental molecular cloud (typically several tenths of a $\mathrm{km} \mathrm{s}^{-1}$ ). The Hyades open cluster in particular is especially suitable and primarily has been used for detailed astrophysical studies because of its (1) proximity (mean distance $\sim 45 \mathrm{pc}$ ), also giving rise to several other advantages such as relatively bright stars and negligible interstellar reddening and extinction, (2) large 


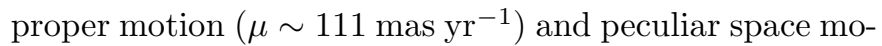
tion $\left(\sim 35 \mathrm{~km} \mathrm{~s}^{-1}\right.$ with respect to its own local standard of rest), greatly facilitating both proper motion- and radial velocity-based membership determinations, and (3) varied stellar content $(\sim 400$ known members, among which are white dwarfs, red giants, mid-A stars in the turn-off region, and numerous main sequence stars, at least down to $\sim 0.10 M_{\odot} \mathrm{M}$ dwarfs). Its proximity, however, has also always complicated astrophysical research: the tidal radius of $\sim 10 \mathrm{pc}$ results in a significant extension of the cluster on the sky $\left(\sim 20^{\circ}\right)$ and, more importantly, a significant depth along the line of sight. As a result, the precise definition and location of the main sequence and turn-off region in the Hertzsprung-Russell (HR) diagram, and thereby, e.g., accurate knowledge of the Helium content and age of the cluster, has always been limited by the accuracy and reliability of distances to individual stars. Unfortunately, the distance of the Hyades is such that ground-based parallax measurements, such as the Yale programme (e.g., van Altena et al. 1995), have never been able to settle "the Hyades distance problem" definitively.

The above situation improved dramatically with the publication of the Hipparcos and Tycho Catalogues (ESA 1997). In 1998, Perryman and collaborators (hereafter P98) published a seminal paper in which they presented the Hipparcos view of the Hyades. P98 studied the threedimensional spatial and velocity distribution of the members, the dynamical properties of the cluster, including its overall potential and density distribution, and its $\mathrm{HR}$ diagram and age. At the mean distance of the cluster ( $D \sim 45 \mathrm{pc}$ ), a typical Hipparcos parallax uncertainty of 1 mas translates into a distance uncertainty of $D^{2} / 1000 \sim$ 2 pc. Because this uncertainty compares favorably with the tidal radius of the Hyades $(\sim 10 \mathrm{pc})$, the Hipparcos distance resolution is sufficient to study details such as mass segregation (Sect. 7.1 in P98). Uncertainties in absolute magnitudes, on the other hand, are still dominated by Hipparcos parallax errors $(\gtrsim 0.10 \mathrm{mag})$ and not by photometric errors ( $\lesssim 0.01 \mathrm{mag}$; Sect. 9.0 in P98).

Kinematic modelling of collective stellar motions in moving groups can yield improved parallaxes for individual stars from the Hipparcos proper motions (e.g., Dravins et al. 1997, 1999; de Bruijne 1999b; hereafter B99b). Such parallaxes, called "secular parallaxes", are more precise than Hipparcos trigonometric parallaxes for individual Hyades as the relative proper motion accuracy is effectively $\sim 3$ times larger than the relative Hipparcos parallax accuracy. P98 discuss secular parallaxes for the Hyades (their Sect. 6.1 and Figs. 10-11), but only in view of their statistical consistency with the trigonometric parallaxes. Improved HR diagrams, based on Hipparcos secular parallaxes, have been published on several occasions, but these diagrams merely served as external verification of the quality and superiority of the secular parallaxes (e.g., Madsen 1999; B99b). Narayanan \& Gould (1999a,b) derived secular parallaxes for the Hyades, but used these only to study the possible presence and size of systematic errors in the Hipparcos data. Although narrow main sequences are readily observable for distant clusters, the absolute calibration of the HR diagram of such groups is often uncertain due to their poorly determined distances and the effects of interstellar reddening and extinction. The latter problems are alleviated significantly for nearby clusters, but a considerable spread in the location of individual members in the HR diagram is introduced as a result of their resolved intrinsic depths.

Hipparcos secular parallaxes for Hyades members provide a unique opportunity to simultaneously obtain a welldefined and absolutely calibrated HR diagram. In this paper we derive secular parallaxes for the Hyades using a slightly modified version of the procedure described by B99b (Sect. 2). Sections 3 and 4 discuss the space motion and velocity dispersion of the Hyades, as well as membership of the cluster, respectively. The secular parallaxes are derived and validated in Sects. 5 and 6 . The validation includes a detailed investigation of the velocity structure of the cluster and of the presence of small-angular-scale correlations in the Hipparcos data. The three-dimensional spatial structure of the Hyades, based on secular parallaxes, is discussed briefly in Sect. 7. Readers who are primarily interested in the secular parallax-based colourabsolute magnitude and HR diagrams can turn directly to Sect. 8; we analyze these diagrams in detail, and also address related issues such as the transformation from observed colours and magnitudes to effective temperatures and luminosities, in Sects. 8-10. Section 11 summarizes and discusses our findings. Appendices A and B present observational data and discuss details of the derivation of fundamental stellar parameters for the Hyades red giants and for the $\delta$ Scuti pulsator $\theta^{2}$ Tau.

\section{History, outline, and revision of the method}

\subsection{History}

We define a moving group (or cluster) as a set of stars which share a common space motion $\mathbf{v}$ to within the internal velocity dispersion $\sigma_{v}$. The canonical formula, based on the classical moving cluster/convergent point method, to determine secular parallaxes $\pi_{\text {sec }}$ from proper motion vectors $\mu$, neglecting the internal velocity dispersion, reads (e.g., Bertiau 1958):

$\pi_{\mathrm{sec}}=\frac{A|\mu|}{|\mathbf{v}| \sin \lambda}$

where $A \equiv 4.740470446 \mathrm{~km} \mathrm{yr} \mathrm{s}^{-1}$ is the ratio of one astronomical unit in kilometers to the number of seconds in one Julian year (ESA 1997, vol. 1, Table 1) and $\lambda$ is the angular distance between a star and the cluster apex. We express parallaxes in units of mas (milli-arcsec) and proper motions in units of mas $\mathrm{yr}^{-1}$.

The derivation of secular parallaxes for Hyades (or Taurus cluster) members from proper motions and/or radial velocities using Eq. (1) dates back to at least Boss (1908; cf. Smart 1939; see Turner et al. 1994 for a review). 
Several authors have criticized such studies for various reasons (e.g., Seares 1945; Brown 1950; Upton 1970; Hanson 1975; cf. Cooke \& Eichhorn 1997); the ideal method simultaneously determines the individual parallaxes and the cluster bulk motion, as well as the corresponding velocity dispersion tensor, from the observational data. Murray \& Harvey (1976) developed such a procedure, and applied it to subsets of Hyades members. Zhao \& Chen (1994) presented a maximum likelihood method for the simultaneous determination of the mean distance (and dispersion) and kinematic parameters (bulk motion and velocity dispersion) of moving clusters, and also applied it to the Hyades. Cooke \& Eichhorn (1997) presented a method for the simultaneous determination of the distances to Hyades and the cluster bulk motion.

\subsection{Outline}

The Hipparcos data recently raised renewed interest in secular parallaxes. Dravins et al. (1997) developed a maximum likelihood method to determine secular parallaxes ${ }^{1}$ based on Hipparcos positions, trigonometric parallaxes, and proper motions, taking into account the correlations between the astrometric parameters (cf. Sect. 2 in B99b). The algorithm assumes that the space velocities of the $n$ cluster members follow a three-dimensional Gaussian distribution with mean $\mathbf{v}$, the cluster space motion, and standard deviation $\sigma_{v}$, the (isotropic) one-dimensional internal velocity dispersion; the three components of $\mathbf{v}$ correspond to the ICRS equatorial Cartesian coordinates $x, y$, and $z$ (ESA 1997, vol. 1, Sect. 1.5.7). The model has $3+1+n$ unknown parameters: $\mathbf{v}, \sigma_{v}$, and the $n$ secular parallaxes.

After maximizing the likelihood function, one can determine the non-negative model-observation discrepancy parameter $g$ for each star (Eq. (8) in B99b). As the $g$ 's are approximately distributed as $\chi^{2}$ with 2 degrees of freedom (Dravins et al. 1997; B99b; cf. Lindegren et al. 2000; Makarov et al. 2000), $g>9$ is a suitable criterion for detecting outliers. Therefore, the procedure can be iterated by rejecting deviant stars (e.g., undetected close binaries or non-members) and subsequently re-computing the maximum likelihood solution, until convergence is achieved in the sense that all remaining stars have $g \leq 9$. By definition, the maximum likelihood estimate of the velocity dispersion $\sigma_{v}$ decreases during this process. Monte Carlo simulations show that this iterative procedure can lead to underestimated values of $\sigma_{v}$ by as much as $0.15 \mathrm{~km} \mathrm{~s}^{-1}$ for Hyades-like groups (B99b).

\subsection{Revision}

A wealth of ground-based radial velocity information was accumulated for the Hyades over the last century. The cluster has an extent on the sky that is large enough

1 The aim of Dravins' investigations is the derivation of astrometric radial velocities. Spectroscopic radial velocities are therefore not used in their modelling. to allow an accurate derivation of its three-dimensional space motion based on proper motion data exclusively (e.g., de Bruijne 1999a,b; Hoogerwerf \& Aguilar 1999). We nonetheless decided to modify the maximum likelihood procedure (Sect. 2.2) so as to enforce global consistency between the maximum likelihood estimate of the radial component of the cluster space motion and the spectroscopic radial velocity data as a set. Therefore, we multiplied the astrometric likelihood merit function $\mathcal{L}$ (Eq. (6) in $\mathrm{B} 99 \mathrm{~b})$ by a radial velocity penalty factor:

$$
\mathcal{L} \longrightarrow \mathcal{L} \cdot \exp \left(\frac{\Delta^{2}}{2 \sigma_{\Delta}^{2}}\right),
$$

where $\Delta$ is the median value of $\left(v_{\text {rad }}-v_{\text {rad,pred }}\right) /$ $\left(\sigma_{v_{\text {rad }}}^{2}+\sigma_{v}^{2}\right)^{1 / 2}$, computed over all stars with a (reliable) radial velocity (Sect. 3.2), where $v_{\text {rad,pred }}=v_{x} \cos \alpha \cos \delta+$ $v_{y} \sin \alpha \cos \delta+v_{z} \sin \delta$, and $(\alpha, \delta)$ denote the equatorial coordinates of a star. The quantity $\sigma_{\Delta}$ is the allowed inconsistency in $\Delta$. We choose $\sigma_{\Delta}=0.5$, which corresponds to $\sim 0.25 \mathrm{~km} \mathrm{~s}^{-1}$ when expressed in terms of the median effective radial velocity uncertainty $\left(\sigma_{v_{\mathrm{rad}}}^{2}+\sigma_{v}^{2}\right)^{1 / 2} \sim$ $0.50 \mathrm{~km} \mathrm{~s}^{-1}$ (Sect. 3.2).

In order to work around the $\sigma_{v}$ bias mentioned in Sect. 2.2, we decided to introduce a second modification of the original procedure. This change involves the decoupling of the determination of the cluster motion plus velocity dispersion (Sect. 3) from the determination of the secular parallaxes and goodness-of-fit parameters $g$ given the cluster velocity and dispersion (Sect. 5; cf. Narayanan \& Gould 1999a,b). We thus reduce the dimensionality of the problem from $3+1+n$ to $n$ (cf. Sect. 3 ). However, the $n$-dimensional maximum likelihood problem of finding $n$ secular parallaxes and goodness-of-fit parameters $g$ for a given space motion and velocity dispersion simplifies directly to $n$ independent one-dimensional problems (Sect. 2 in B99b). This leads to three additional advantages: (1) it reduces the computational complexity of the problem; (2) it allows an a posteriori decision on the $g$ rejection limit (Sect. 2.2); and (3) it allows a treatment on the same footing of stars lacking trigonometric parallax information, such as Hyades selected from the Tycho-2 catalogue (Sect. 4.3). In practice, the analysis of such stars only requires the reduction of the dimensionality of the vector of observables $\boldsymbol{a}_{\text {Hip }}$ and the corresponding vector $\boldsymbol{c}$ and matrices $\mathrm{C}_{\text {Hip }}$ and $\mathrm{D}$ from 3 to 2 and $3 \times 3$ to $2 \times 2$, respectively, through suppression of the first component (see Sect. 2 in B99b).

\section{Space motion and velocity dispersion}

The procedure outlined in Sects. $2.2-2.3$ is based on two basic assumptions, namely that (1) the cluster velocity field is known accurately, and (2) the astrometric measurements of the stars correctly reflect their true space motions. Assumption (2) can be safely met by excluding from the analysis (close) multiple systems, spectroscopic binaries, etc. (Sect. 4.3). Assumption (1) requires a careful analysis, to which we will return in Sect. 6.4. For the 


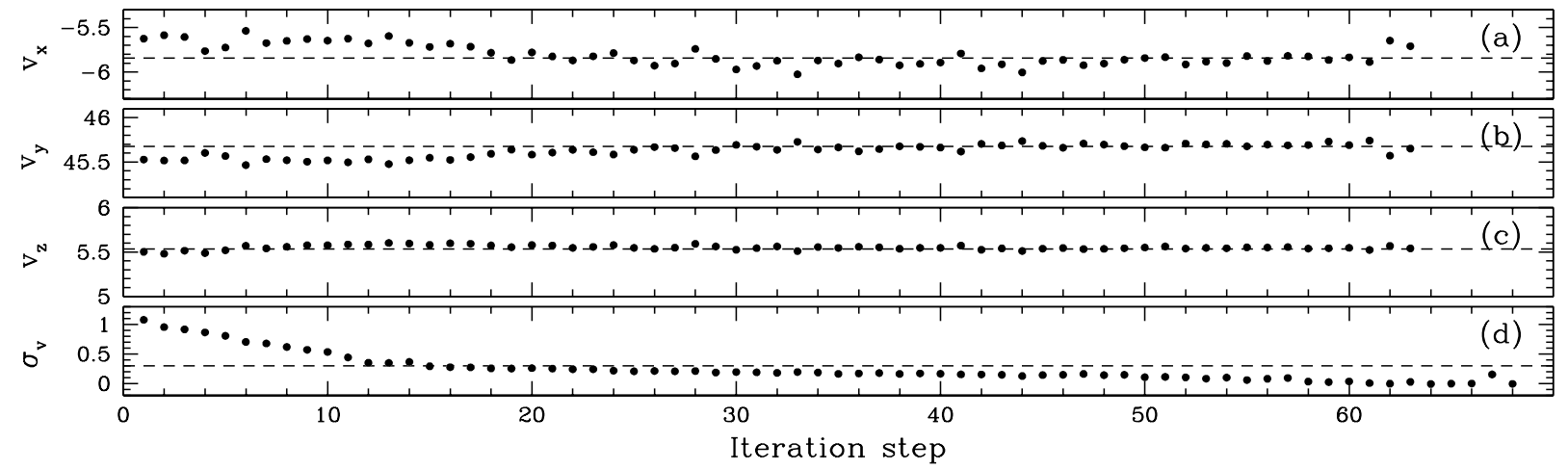

Fig. 1. Panels $\mathbf{a}-\mathbf{c}$ ) the evolution of the cluster space motion components $v_{x}, v_{y}$, and $v_{z}$ (in $\mathrm{km} \mathrm{s}^{-1}$ ) in the equatorial Cartesian ICRS frame during the rejection of stars in the revised iterative procedure using $\sigma_{v}=0.30 \mathrm{~km} \mathrm{~s}^{-1}, \sigma_{\Delta}=0.5$, and a $g$ rejection limit of 9 (Sects. 2.3 and 3.2). The dots show the values of the space motion components at a given iteration step. The dashed lines denote the median values of the space motion components: $\left(v_{x}, v_{y}, v_{z}\right)=(-5.84,45.68,5.54) \mathrm{km} \mathrm{s}^{-1}$. Panel d) the evolution of the velocity dispersion $\sigma_{v}$ during the rejection of stars in the unrevised procedure using a $g$ rejection limit of 9 (Sects. 2.2 and 3.1). The dots show the median values of the velocity dispersion at a given iteration step. The discontinuity of the slope of the relation exhibited by the dots around step $\sim 12$ coincides with the physical (one-dimensional) velocity dispersion of the Hyades $\left(\sim 0.30 \mathrm{~km} \mathrm{~s}^{-1}\right.$; dashed line)

moment, it suffices to say that there exist neither conclusive observational evidence nor theoretical predictions that the velocity field of, at least the central region of, the Hyades cluster is non-Gaussian with an anisotropic velocity dispersion.

We opt to define the space motion and velocity dispersion of the Hyades based on a well-defined set of secure members. P98 used the Hipparcos positions, parallaxes, and proper motions, complemented with ground-based radial velocities if available, to derive velocities for individual stars in order to establish membership based on the assumption of a common space motion. P98 identified 218 candidate members, all of which are listed in their Table 2 (i.e., Col. (x) is "1" or "?"). We take these stars, and exclude all objects without radial velocity information (i.e., Col. (p) is "*") and all (candidate) close binaries (i.e., either Col. (s) is "SB" (spectroscopic binary) or Col. (u) is one of "G O V X S"; see ESA 1997 for the definition of the Hipparcos astrometric multiplicity fields H59 and H61). This leaves 131 secure single members with high-quality astrometric and radial velocity information ${ }^{2,3}$.

${ }^{2}$ Contrary to what had been communicated, the 26 "new Coravel radial velocities" in P98's Table 2 (Col. (r) is "24") do not include the standard zero-point correction of $+0.40 \mathrm{~km} \mathrm{~s}^{-1}$ (Sect. 3.2 in P98; finding confirmed by J.-C. Mermilliod through private communication).

3 As described in P98, the Griffin et al. radial velocities (Col. (r) is " 1 ") for main sequence stars in their Table 2 have to be corrected according to Gunn et al.'s (1988) Eq. (12), but accounting for a sign error (Gunn's Eq. (12) should read: $v_{\text {meas }}-v_{\text {true }}=\ldots$ instead of $\left.v_{\text {true }}-v_{\text {meas }}=\ldots\right)$ : $v_{\text {rad,corrected }}=v_{\text {rad }}-q(V)-0.5 \mathrm{~km} \mathrm{~s}^{-1}$ for $V>6.0 \mathrm{mag}$ and $v_{\text {rad,corrected }}=v_{\text {rad }}-0.5 \mathrm{~km} \mathrm{~s}^{-1}$ for $V \leq 6.0 \mathrm{mag}$, where $q(V)=0.44-70010^{-0.4 \cdot V}$. The seemingly large discontinuity $\left(\sim 2.3 \mathrm{~km} \mathrm{~s}^{-1}\right)$ at $V=6 \mathrm{mag}$ in this correction is academic for the Hyades as there are no main sequence members brighter than this magnitude.

\subsection{Velocity dispersion}

We start the unrevised procedure (Sect. 2.2) for the abovedescribed sample of 131 stars using a $g$ rejection limit of 9. Figure 1 (panel d) shows the evolution of the maximum likelihood value derived for $\sigma_{v}$ while rejecting stars. The estimated velocity dispersion decreases rapidly, more-orless linearly, from $\sim 1 \mathrm{~km} \mathrm{~s}^{-1}$ initially to $\sim 0.30 \mathrm{~km} \mathrm{~s}^{-1}$ in the first $\sim 11$ steps. Previous studies of the Hyades cluster show that its physical (one-dimensional) velocity dispersion is $\sim 0.30 \mathrm{~km} \mathrm{~s}^{-1}$ (Gunn et al. 1988: $0.23 \pm 0.05 \mathrm{~km} \mathrm{~s}^{-1}$; Zhao \& Chen 1994: 0.37 $\pm 0.04 \mathrm{~km} \mathrm{~s}^{-1}$; Dravins et al. 1997: $0.25 \pm 0.04 \mathrm{~km} \mathrm{~s}^{-1}$; P98: 0.20-0.40 km s${ }^{-1}$; Narayanan \& Gould 1999a,b: $0.32 \pm 0.04 \mathrm{~km} \mathrm{~s}^{-1}$; Lindegren et al. 2000: $0.31 \pm 0.02 \mathrm{~km} \mathrm{~s}^{-1}$; Makarov et al. 2000: 0.32 $\mathrm{km} \mathrm{s}^{-1}$ ). From step $\sim 12$ onwards, the maximum likelihood dispersion estimate decreases, again more-or-less linearly but much more gradually, to $\sim 0 \mathrm{~km} \mathrm{~s}^{-1}$ in step $\sim 60-70$. Not surprisingly, the corresponding evolution of the space motion shows an unwanted trend beyond step $~ 12$ (not shown): the unrevised method is forced to search for a maximum likelihood solution which has a velocity dispersion that is smaller than the physical value.

Given the Hyades space motion (or convergent point; Sect. 3.2), a semi-independent ${ }^{4}$ estimate of the internal velocity dispersion $\sigma_{v}$ can be derived through a so-called $\mu_{\perp}$-component analysis (Sect. 20 in Blaauw 1946; Sect. 7.2 in P98; Sect. 4.2 in B99b; Lindegren et al. 2000). The $\mu_{\perp}$ proper motion components are directed perpendicular to the great circle joining a star and the apex, and as such, by definition, exclusively represent peculiar motions

\footnotetext{
${ }^{4}$ Kinematic member selection requires an a priori estimate of the expected velocity dispersion in the cluster. The stars used in this analysis were selected as members by P98 under the assumption that the cluster velocity dispersion is small compared to the typical measurement error of a stellar velocity.
} 
Table 1. Comparison of the Hyades space motion of different studies: this study $\left(\left[\sigma_{v}, \sigma_{\Delta}\right]\right.$, where $\sigma_{v}=0.20,0.30$, or $0.40 \mathrm{~km} \mathrm{~s}{ }^{-1}$ and $\sigma_{\Delta}=0.1$ or 0.5 ; the default is $[0.30,0.50]$ ), Perryman et al. (1998; P98 [134/180] for the 134/180 stars within 10/20 pc of the cluster center), Narayanan \& Gould (1999a; NG99a), Dravins et al. (1997; D97), and Lindegren et al. (2000; LMD00). The components of the cluster space motion $\mathbf{v}$ in the equatorial Cartesian ICRS coordinate system are $v_{x}, v_{y}$, and $v_{z}$ (in $\mathrm{km} \mathrm{s}^{-1}$ ). The coordinate system $\left(v_{r}, v_{\perp}, v_{\|}\right)$is described in Sect. 3.2. The convergent point $(\alpha, \delta)_{\mathrm{cp}}$ is given in equatorial coordinates (in the ICRS system in degrees). The Hyades convergent point coordinates are strongly correlated; a typical value for the correlation coefficient $\rho$ between $\alpha_{\mathrm{cp}}$ and $\delta_{\mathrm{cp}}$ is $\rho \sim-0.8$ (Sect. 4.1 and Fig. 4 in de Bruijne 1999a)

\begin{tabular}{lccccccccccc}
\hline \hline Study & $v_{x}$ & $\sigma_{v_{x}}$ & $v_{y}$ & $\sigma_{v_{y}}$ & $v_{z}$ & $\sigma_{v_{z}}$ & $v_{r}$ & $v_{\perp}$ & $v_{\|}$ & $\alpha_{\mathrm{cp}}$ & $\delta_{\mathrm{cp}}$ \\
\hline$[0.30,0.5]$ & -5.84 & 0.26 & 45.68 & 0.11 & 5.54 & 0.07 & 39.48 & 0.00 & 24.35 & 97.29 & 6.86 \\
{$[0.30,0.1]$} & -5.74 & 0.10 & 45.67 & 0.08 & 5.58 & 0.04 & 39.52 & -0.00 & 24.25 & 97.16 & 6.91 \\
{$[0.20,0.5]$} & -5.77 & 0.10 & 45.64 & 0.05 & 5.59 & 0.07 & 39.49 & -0.03 & 24.25 & 97.21 & 6.93 \\
{$[0.40,0.5]$} & -5.99 & 0.18 & 45.73 & 0.09 & 5.52 & 0.05 & 39.46 & -0.03 & 24.50 & 97.46 & 6.83 \\
P98 [134] & -6.28 & 0.20 & 45.19 & 0.20 & 5.31 & 0.20 & 38.82 & -0.02 & 24.56 & 97.91 & 6.66 \\
P98 [180] & -6.32 & 0.20 & 45.24 & 0.20 & 5.30 & 0.20 & 38.84 & -0.02 & 24.62 & 97.96 & 6.61 \\
NG99a & -5.70 & 0.20 & 45.62 & 0.11 & 5.65 & 0.08 & 39.51 & -0.06 & 24.17 & 97.12 & 7.01 \\
D97 & -6.07 & 0.13 & 45.77 & 0.36 & 5.53 & 0.11 & 39.47 & -0.06 & 24.59 & 97.55 & 6.83 \\
LMD00 & -5.90 & 0.13 & 45.65 & 0.34 & 5.56 & 0.10 & 39.44 & -0.05 & 24.38 & 97.36 & 6.89 \\
\hline \hline
\end{tabular}

$\left(\Delta_{v, \perp} ;\right.$ one-dimensional, in $\left.\mathrm{km} \mathrm{s}^{-1}\right)$ and observational er$\operatorname{rors}\left(\Delta_{\mu_{\perp}} ;\right.$ in $\left.\operatorname{mas} \mathrm{yr}^{-1}\right)$ :

$\mu_{\perp}=A^{-1} \pi \Delta_{v, \perp}+\Delta_{\mu_{\perp}} \Longrightarrow \overline{\mu_{\perp}^{2}}=\overline{\left(A^{-1} \pi \Delta_{v, \perp}\right)^{2}}+\overline{\Delta_{\mu_{\perp}}^{2}},(3)$

where the step follows from the statistical independence of the peculiar motions and the observed proper motion errors. Upon using $\pi=21.58 \operatorname{mas}^{5}$ ( $D=46.34$ pc; P98), and calculating $\mu_{\perp}$ and $\Delta_{\mu_{\perp}}$ from the Hipparcos positions and proper motions using the maximum likelihood apex $\left(\alpha_{\mathrm{cp}}, \delta_{\mathrm{cp}}\right)=\left(97^{\circ} 29,6.86\right)$ (Table 1; Sect. 3.2), it follows that $\left(\overline{\Delta_{v, \perp}^{2}}\right)^{1 / 2} \sim 0.20-0.40 \mathrm{~km} \mathrm{~s}^{-1}$, where the precise value of this quantity depends on the details of the selection and subdivision of the stellar sample (Table 2). The abovementioned range is consistent with our assumed value of $0.30 \mathrm{~km} \mathrm{~s}^{-1}$. We therefore decided to take $\sigma_{v}=0.30 \mathrm{~km} \mathrm{~s}^{-1}$ fixed in the remainder of this study, i.e., we reduce the dimensionality of the problem from $3+1+n$ to $3+n$ (Sect. 2.3).

\subsection{Space motion}

Our next step is to start the revised procedure (Sect. 2.3) for the same sample of 131 stars, but take $\sigma_{v}=$ $0.30 \mathrm{~km} \mathrm{~s}^{-1}$ and $\sigma_{\Delta}=0.5$. We exclude multiple systems without a known systemic (or center-of-mass or $\gamma$-) velocity, as well as objects with a variable radial velocity, in the calculation of the penalty factor (Eq. (2); i.e., all stars with a \#-sign preceding Col. (q) in P98's Table 2). Figure 1 shows the evolution of the maximum likelihood estimates of the space motion components while rejecting stars; we derive $\left(v_{x}, v_{y}, v_{z}\right)=(-5.84 \pm 0.26,45.68 \pm$ $0.11,5.54 \pm 0.07) \mathrm{km} \mathrm{s}^{-1}$. Table 1 shows the results of varying $\sigma_{\Delta}$ and $\sigma_{v}$. Changing $\sigma_{\Delta}$, for example, from 0.5 to 0.1 at fixed $\sigma_{v}=0.30 \mathrm{~km} \mathrm{~s}^{-1}$ yields a set of secular parallaxes (Sect. 5) which differ systematically in the sense $\left\langle\pi_{\mathrm{sec}, \sigma_{\Delta}=0.1}-\pi_{\mathrm{sec}, \sigma_{\Delta}=0.5}\right\rangle \sim+0.08$ mas, independent of visual magnitude. We take $\left(v_{x}, v_{y}, v_{z}\right)=$ $(-5.84,45.68,5.54) \mathrm{km} \mathrm{s}^{-1}$ fixed in the remainder of this

\footnotetext{
${ }^{5}$ Individual secular parallaxes (Sect. 5) give identical results.
}

study, i.e., we reduce the dimensionality of the problem, now from $3+n$ to $n$.

Table 1 compares the space motions found by us with results derived by $\mathrm{P}^{6} 8^{6}$, Dravins et al. (1997), Narayanan \& Gould (1999a), and Lindegren et al. (2000) from Hipparcos data. We refrain from comparing our values to pre-Hipparcos results (e.g., Schwan 1991; Zhao \& Chen 1994; Cooke \& Eichhorn 1997), as these are (possibly) influenced by fundamental uncertainties in the preHipparcos proper motion reference frames (the Hipparcos positions and proper motions are absolute, and are given in the Hipparcos ICRS inertial reference frame; cf. Sect. 4 in P98). Table 1 also compares the different space motions in the $\left(v_{r}, v_{\perp}, v_{\|}\right)$coordinate system, which is oriented such that the $v_{r}$-axis is along the radial direction of the cluster center, which is (arbitrarily) defined as $(\alpha, \delta)_{\text {center }}=\left(4^{\mathrm{h}} 26^{\prime} 32^{\prime \prime}, 17^{\circ} 13.3\right)(\mathrm{J} 2000.0)$, the $v_{\perp^{-}}$ axis is along the direction perpendicular to the cluster proper motion in the plane of the sky, and the $v_{\|^{-}}$ axis is parallel to the cluster proper motion in the plane of the sky. We conclude that our space motion is consistent with the values derived by Dravins et al., P98, Narayanan \& Gould, and Lindegren et al.; our radial motion agrees very well with the Dravins et al., Narayanan \& Gould, and Lindegren et al. values, whereas our tangential motion perfectly agrees with Lindegren et al. and lies between the Narayanan \& Gould value on the one hand and the P98 and Dravins et al. values on the other hand. The radial components of the P98 space motions (38.82-38.84 $\mathrm{km} \mathrm{s}^{-1}$ ) deviate significantly (at the level

${ }^{6}$ Whereas our space cluster motion(s) and the values listed by Dravins et al. and Narayanan \& Gould correspond to the arithmetic mean value of individual motions of (a given set of) stars, P98 lists mass-weighted mean values of individual velocities. We investigated the effect of weighing the individual motions by stellar mass, and found the difference between the final cluster space motions to be generally less than $0.10 \mathrm{~km} \mathrm{~s}^{-1}$ in each coordinate; we therefore conservatively assume that quoting a 0.20 instead of a $0.10 \mathrm{~km} \mathrm{~s}^{-1}$ error on the P98 space motion components "absorbs" this uncertainty. 
of $\sim 0.70 \mathrm{~km} \mathrm{~s}^{-1}$ ) from all other values in Table 1 (cf. Sect. 4.2 in Narayanan \& Gould 1999a). Unfortunately, the mean spectroscopically determined radial velocity of the Hyades cluster is not well defined; Detweiler et al. (1984), for example, find $39.1 \pm 0.2 \mathrm{~km} \mathrm{~s}^{-1}$, but their Table 1 gives an overview of previous determinations which show a discouragingly large spread (cf. Gunn et al. 1988). Figure 2 shows, for the 131 secure single members, the distribution of observed radial velocities (left), the distribution of observed minus predicted radial velocities (Sect. 2.3) given the cluster space motion (middle), and the properly normalized distribution of observed minus predicted radial velocities (right; taking into account a velocity dispersion of $\left.\sigma_{v}=0.30 \mathrm{~km} \mathrm{~s}^{-1}\right)$. The distribution of observed radial velocities is not symmetric but skewed towards lower $v_{\text {rad,obs }}$ values; the median $v_{\text {rad,obs }}$ value $\left(38.60 \mathrm{~km} \mathrm{~s}^{-1}\right)$ is $0.66 \mathrm{~km} \mathrm{~s}^{-1}$ larger than the straight mean of the observed $v_{\text {rad,obs }}$ values $\left(37.94 \mathrm{~km} \mathrm{~s}^{-1}\right)$. The large spread and skewness in the distribution of observed radial velocities are caused by the perspective effect, which is significant for the Hyades due to its large extent on the sky (Sect. 1). The perspective effect has been removed in the middle and right panels of Fig. 2. The middle panel shows that the radial component of our space motion $\left(39.48 \mathrm{~km} \mathrm{~s}^{-1}\right)$ yields an acceptable $v_{\text {rad,obs }}-v_{\text {rad,pred }}$ distribution. The deviation between the predicted zero-mean unit-variance Gaussian and the observed histogram in the right panel is possibly caused by (1) the presence of a few non-members (and possibly some not-detected close binaries), (2) a slightly underestimated cluster velocity dispersion, and/or (3) underestimated $v_{\text {rad,obs }}$ errors. The histogram and Gaussian prediction would agree, given the $v_{\text {rad,obs }}$ errors, if $\sigma_{v}$ is increased to $\sim 0.80-0.90 \mathrm{~km} \mathrm{~s}^{-1}$, or, given $\sigma_{v}=0.30 \mathrm{~km} \mathrm{~s}^{-1}$, if the individual random $v_{\text {rad,obs }}$ errors are increased by an amount of $\sim 0.50-0.60 \mathrm{~km} \mathrm{~s}^{-1}$. While the first possibility seems highly unlikely (Sect. 3.1; cf. Gunn et al. 1988), the required "extra radial velocity uncertainty" is not unreasonable, given it is of the same order of magnitude as the (poorly determined) non-physical zero-point shifts usually adopted in radial velocity studies (e.g., Gunn et al. 1988; cf. Sects. 3.2 and 7.2 in P98).

\section{Membership}

Having determined the Hyades space motion and velocity dispersion, we are in a position to discuss membership.

\subsection{Hipparcos: Perryman et al. (1998) candidates}

The Hipparcos Catalogue contains 118218 entries which are homogeneously distributed over the sky. The catalogue is complete to $V \sim 7.3 \mathrm{mag}$, and has a limiting magnitude of $V \sim 12.4 \mathrm{mag}$. In the case of the Hyades, special care was taken to optimize the number of candidate members in the Hipparcos target list. As a result, the Hipparcos Input Catalogue (Turon et al. 1992) contains $\sim 240$ candidate Hyades members in the field $2^{\mathrm{h}} 15^{\mathrm{m}}<\alpha<6^{\mathrm{h}} 5^{\mathrm{m}}$ and $-2^{\circ}<\delta<35^{\circ}$ (Sect. 3.1 in P98). P98 considered all 5490 Hipparcos entries in this field for membership, and

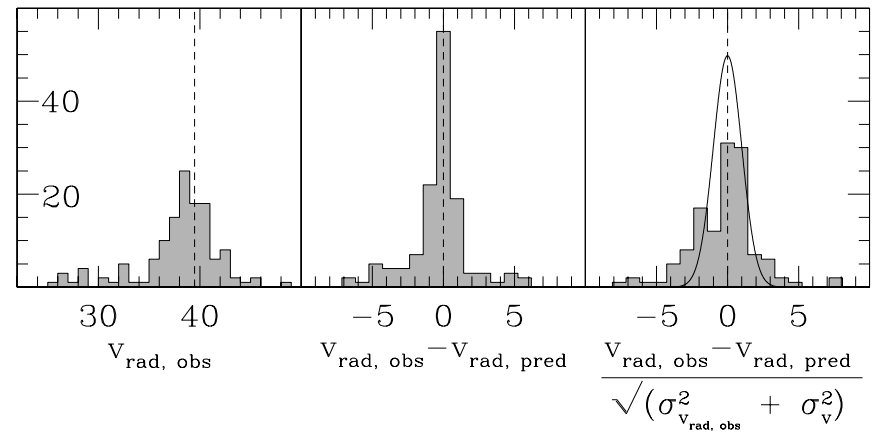

Fig. 2. Observed ( $\left.v_{\text {rad,obs }}\right)$ and predicted ( $v_{\text {rad,pred }}$; Sect. 2.3$)$ radial velocities for 131 secure members (Sect. 3). Left: distribution of observed radial velocities; the dashed line denotes the radial component of the Hyades cluster motion derived in this study $\left(39.48 \mathrm{~km} \mathrm{~s}^{-1}\right)$ using $\sigma_{v}=0.30 \mathrm{~km} \mathrm{~s}^{-1}$ and $\sigma_{\Delta}=0.5$ $([0.30,0.5]$ in Table 1$)$. The spread and skewness of the distribution are caused by the perspective effect. Middle: distribution of observed minus predicted radial velocities; 71/60 stars have negative/positive $v_{\text {rad,obs }}-v_{\text {rad,pred }}$. Right: normalized distribution of observed minus predicted radial velocities, taking into account a velocity dispersion of $\sigma_{v}=0.30 \mathrm{~km} \mathrm{~s}^{-1}$. The black curve is a properly scaled zero-mean unit-variance Gaussian; the mismatch between the observations and prediction can be due to non-members and/or undetected close binaries, an underestimated velocity dispersion, underestimated radial velocity errors, or a combination of these effects (Sect. 3.2). Five stars fall outside the plotted range

Table 2. Statistics of the $\mu_{\perp}$ proper motion components for the 63 brightest (spectral type $=\mathrm{SpT} \leq \mathrm{G} 5$ ) high-fidelity ( $g \equiv g_{\text {Hip }} \leq 9$; Sect. 5) single P98 members (Col. (s) in P98's Table 2 is not "SB"), using $\left(\alpha_{\mathrm{cp}}, \delta_{\mathrm{cp}}\right)=\left(97^{\circ} .29,6.86\right)$ (see Eq. (3) and Sect. 3.1). Hyades main sequence members with spectral types later than $\sim$ G5 have modest Hipparcos proper motion accuracies due to their faint magnitudes $(V \gtrsim 8.5 \mathrm{mag}$; e.g., Fig. 1 in Hoogerwerf 2000). Results are tabulated for four ranges in spectral type ( $n$ stars from $\mathrm{SpT}_{-}$trough $\left.\mathrm{SpT}_{+}\right)$; the spectral-type averaged value of $\left(\overline{\Delta_{v, \perp}^{2}}\right)^{1 / 2}$ for these 63 stars is $\sim 0.25 \mathrm{~km} \mathrm{~s}^{-1}$

\begin{tabular}{cccccc}
\hline \hline $\mathrm{SpT}_{-}$ & $\mathrm{SpT}_{+}$ & $n$ & $\begin{array}{c}\left(\overline{\mu_{\perp}^{2}}\right)^{1 / 2} \\
\text { mas }\end{array}$ & $\begin{array}{c}\left(\overline{\Delta_{\mu_{\perp}^{2}}^{2}}\right)^{1 / 2} \\
\text { mas }\end{array}$ & $\begin{array}{c}\left(\overline{\Delta_{v, \perp}^{2}}\right)^{1 / 2} \\
\mathrm{~km} \mathrm{~s}^{-1}\end{array}$ \\
\hline $\mathrm{A} 2$ & F0 & 16 & 1.35 & 0.89 & 0.23 \\
F0 & F5 & 16 & 1.71 & 0.88 & 0.31 \\
F5 & F8 & 16 & 1.33 & 0.96 & 0.20 \\
F9 & G5 & 15 & 1.71 & 1.13 & 0.28 \\
\hline \hline
\end{tabular}

ended up with 218 members. The P98 member selection is generous: only very few genuine members, contained in both the Hipparcos Catalogue and the selected field on the sky, have probably not been selected, whereas a number of field stars (interlopers) are likely to be present in their list. P98 distinguish members (197 stars) and possible members (21 stars); the latter do not have measured radial velocities (Col. (x) = "?" in their Table 2 ).

P98 divide the Hyades into four components $(r$ is the three-dimensional distance to the cluster center): (1) a spherical "core" with a $2.7 \mathrm{pc}$ radius and a half-mass radius of $5.7 \mathrm{pc}$; (2) a "corona" extending out to the tidal 
radius $r_{\mathrm{t}} \sim 10 \mathrm{pc}$ (134 stars in core and corona); (3) a "halo" consisting of stars with $r_{\mathrm{t}} \lesssim r \lesssim 2 r_{\mathrm{t}}$ which are still dynamically bound to the cluster (45 stars; e.g., Pels et al. 1975); and (4) a "moving group population" of stars, possibly former members, with $r \gtrsim 2 r_{\mathrm{t}}$ which have similar kinematics to the bound members in the central parts of the cluster (39 stars; e.g., Asiain et al. 1999; cf. Sect. 7 in P98).

The fact that P98 restricted their search to a predefined field on the sky limits knowledge on and completeness of membership, especially in the outer regions of the cluster: the $10 \mathrm{pc}$ tidal radius translates to a cluster diameter of $\sim 25^{\circ}$, whereas the P98 field measures $57^{\circ} .5$ in $\alpha$ and $37^{\circ} 0$ in $\delta$. Although this problem seems minor at first sight, suggesting a solution in the form of simply searching the entire Hipparcos Catalogue for additional (moving group) members, it is daunting in practice: thousands of Hipparcos stars all over the sky have proper motions directed towards the Hyades convergent point (Sect. 4.2 in de Bruijne 1999a). Whereas this in principle means that these stars, in projection at least, are "co-moving" with the Hyades, the identification of physical members of a moving group (or "supercluster") population is not trivial, and requires additional observational data (cf. Sects. 7-8 and Table 6 in P98; Sect. 6.4.2). We therefore restrict ourselves to the P98 field (Sect. 4.2). Section 4.3 discusses the possibility to extend membership down to fainter magnitudes using the Tycho-2 astrometric catalogue.

\subsection{Hipparcos: Additional candidates}

De Bruijne (1999a) and Hoogerwerf \& Aguilar (1999) reanalyzed Hyades membership, based on the refurbished convergent point and Spaghetti method. These studies used Hipparcos data but excluded radial velocity information. The convergent point method uses proper motion data only, confirms membership for 203 of the 218 P98 members (cf. Table A.1), and selects 12 new candidates within $20 \mathrm{pc}$ of the cluster center. The Spaghetti method, using proper motion and parallax data, selects six new candidate members, three of which are in common with the 12 proper motion candidates mentioned above. The Spaghetti method does not confirm 56 P98 members (cf. Table A.1); however, 49 of these are low-probability (i.e., "1-3б") P98 members. Table A.2 lists the 15 new candidates. We defer the discussion of these stars to Sect. 5.2.

\subsection{Tycho-2: Bright binaries and faint candidates}

The Tycho(-1) Catalogue (ESA 1997), which is based on measurements of Hipparcos' starmapper, contains astrometric data for $\sim 1$ million stellar systems with a $\sim 10-20$ times smaller precision than Hipparcos. Its completeness limit is $V \sim 10.5 \mathrm{mag}$. Despite the "inferior astrometric precision", the Tycho positions as a set are superior to similar measurements in any other catalogue of comparable size. The Tycho measurements (median epoch
1991.25) have therefore been used as second epoch material in the construction of a long time-baseline proper motion project, culminating in the Tycho-2 catalogue (Høg et al. 2000a,b; cf. Urban et al. 1998a; Kuzmin et al. 1999). This project uses the Astrographic Catalogue positions, as well as data from 143 other ground-based astrometric catalogues, as first epoch material (median epoch 1904). The Astrographic Catalogue (Débarbat et al. 1988; Urban et al. 1998b) was part of the "Carte du Ciel" project, which envisaged the imaging of the entire sky on 22660 overlapping photographic plates by 20 observatories in different "declination zones". The Tycho-2 catalogue contains absolute proper motions in the Hipparcos ICRS frame for $\sim 2.5$ million stars with a median error of

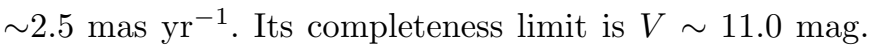
Tycho- 2 contains proper motions for 208 of the 218 P98 candidates; the entries HIP 20440, 20995, and 23205 are (photometrically) resolved binaries in Tycho-2.

The Tycho- 2 proper motions can be used in two ways. First, as a result of the $\sim 4$ year time baseline over which Hipparcos obtained its astrometric data, the proper motions of some multiple systems do not properly reflect their true systemic motions as a result of unrecognized orbital motion (e.g., de Zeeuw et al. 1999; Wielen et al. 1999, 2000; these systems are called " $\Delta \mu$ binaries"). As the long time-baseline Tycho-2 proper motions suffer from this effect to a much smaller extent, they sometimes significantly better represent the true motion of an object than the Hipparcos measurements do. Second, the Tycho2 catalogue can in principle be used to provide membership information for stars beyond the Hipparcos completeness limit, i.e., in the range $7.3 \lesssim V \lesssim 12.4 \mathrm{mag}$ (the Tycho-2 catalogue contains $~ 90000$ stars in P98's Hyades field). The search for new (faint) members is, however, non-trivial. The Hipparcos Catalogue in the field of the Hyades is already relatively complete in terms of (known) members of the cluster (cf. Fig. 2 and Sect. 3.1 in P98), so that the majority of new members is necessarily quite faint $(V \gtrsim 10.0 \mathrm{mag})$. Moreover, most of the Tycho-2 entries have no known radial velocity and/or parallax (although some stars in the Tycho-2 catalogue have Tycho parallax measurements, the typical associated random errors for Hyades fainter than $V \sim 7.3$ mag are similar in size or larger than the expected parallaxes themselves, $\pi \sim 22$ mas). We therefore cannot select (faint) Tycho-2 Hyades members along the lines set out by P98, but must, e.g., follow Hoogerwerf's (2000) method instead. This method, which is based on a convergent point method, selects (faint) stars which (1) are consistent with a given convergent point and the two-dimensional proper motion distribution of a given set of (bright) Hipparcos members, and (2) follow a given main sequence. However, the resulting list of candidate members contains hundreds of falsely identified objects (interlopers), especially in the faint magnitude regime (e.g., Hoogerwerf 2000; cf. de Bruijne 1999a). Reliable suppression of these stars requires at least (yet unavailable) radial velocity and/or 


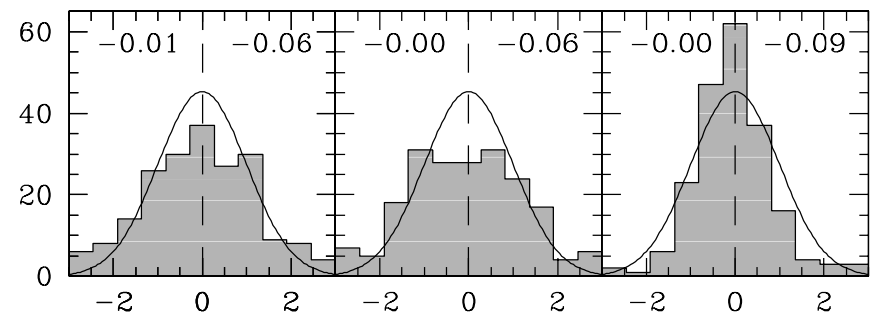

$\left(\pi_{\text {Hip }}-\pi_{\text {sec,Hip }}\right) / \sigma \quad\left(\pi_{\text {Hip }}-\pi_{\text {sec,Tycho-2 }}\right) / \sigma \quad\left(\pi_{\text {sec,Hip }}-\pi_{\text {sec,Tycho-2 }}\right) / \sigma$
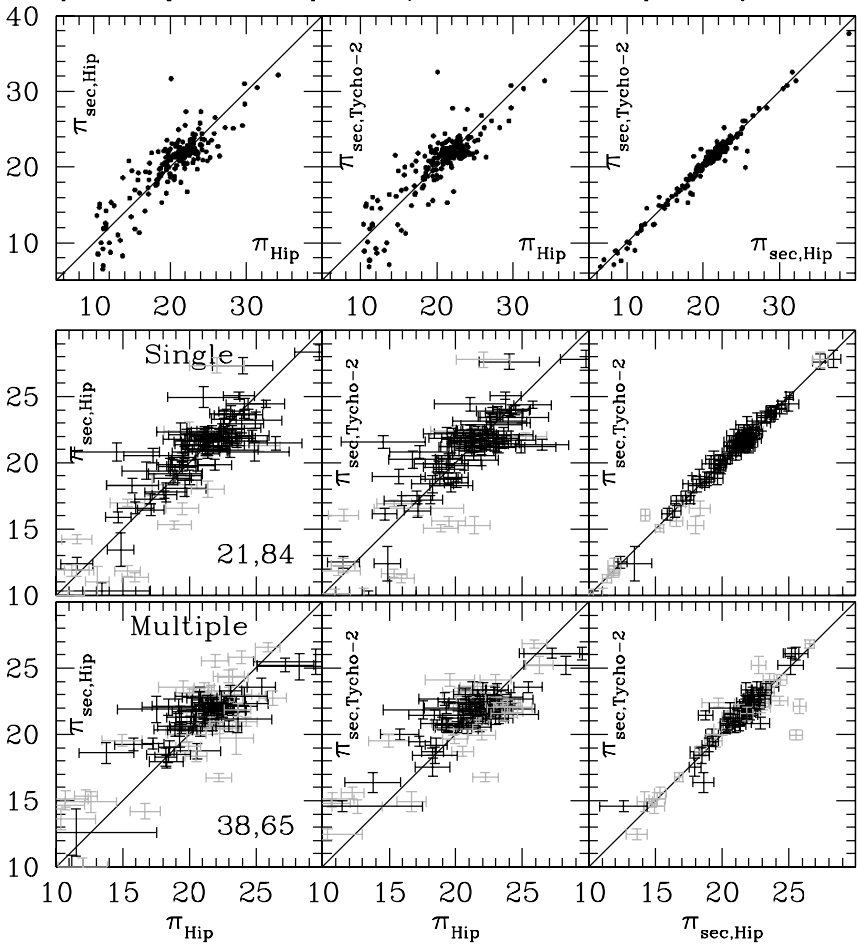

Fig. 3. First and second row: the 208 entries which have Hipparcos trigonometric $\left(\pi_{\mathrm{Hip}}\right)$, Hipparcos secular $\left(\pi_{\mathrm{sec}, \mathrm{Hip}}\right)$, and Tycho-2 secular $\left(\pi_{\text {sec,Tycho-2 }}\right)$ parallaxes (in mas). The top row shows properly normalized difference distributions for $\pi_{\text {Hip }}-\pi_{\text {sec,Hip }}$ (Col. 1), $\pi_{\text {Hip }}-\pi_{\text {sec,Tycho-2 }}$ (Col. 2), and $\pi_{\text {sec }, \text { Hip }}-\pi_{\text {sec,Tycho-2 }}$ (Col. 3$)$. The black curves are zero-mean unit-variance Gaussian distributions. The numbers in the top of each top panel denote the mean (left) and median (right) of the plotted difference. The second row compares the different parallaxes. Third and fourth row: trigonometric and secular parallaxes, and their random errors (Sect. 5.4), for the same stars. The third row compares the different parallaxes for single stars, while the bottom row shows multiple stars (i.e., either Col. (s) in P98's Table 2 is "SB" or "RV", Col. (t) is "H", "I", or "M", or Col. (u) is one of "CGOVXS"). Black symbols have $g_{\text {Hip }} \leq 9$; gray symbols have $g_{\text {Hip }}>9$. The numbers in the left panels indicate the relevant numbers of stars (gray, black). Stars having large goodness-of-fit parameters $g_{\text {Hip }}$ are $\sim 2$ times more likely multiple than low- $g_{\text {Hip }}$ stars (cf. Sect. 3 )

parallax data. We therefore refrain from pursuing this route further in this paper.

\section{Secular parallaxes}

We now determine secular parallaxes for the 218 P98 members and the 15 new candidates discussed in Sect. 4, using the space motion and velocity dispersion found in Sect. $3\left(v_{x}, v_{y}, v_{z}, \sigma_{v}=-5.84,45.68,5.54,0.30 \mathrm{~km} \mathrm{~s}^{-1}\right)$ as constants (Sect. 2.3). This provides, for each proper motion (either Hipparcos or Tycho-2), a secular parallax $\left(\pi_{\text {sec,Hip }}\right.$ or $\left.\pi_{\text {sec,Tycho-2 }}\right)$ and an associated random error $\left(\sigma_{\pi, \mathrm{sec}, \mathrm{Hip}}\right.$ or $\sigma_{\pi, \mathrm{sec}, \text { Tycho-2 }}$; Sect. 5.4) and goodnessof-fit parameter $\left(g_{\text {Hip }}\right.$ or $g_{\text {Tycho-2 }}$; Appendix A). As the Hipparcos and Tycho-2 proper motions are independent measurements, the corresponding secular parallaxes can in principle be averaged, taking the errors into account as weighting factors. It is, however, less clear how to incorporate the goodness-of-fit parameters $g_{\text {Hip }}$ and $g_{\text {Tycho-2 }}$ in the averaging process. We therefore provide both secular parallaxes for all stars and refrain from giving any average value.

\subsection{Hipparcos: Perryman et al. (1998) candidates}

Figure 3 shows a global comparison between the different sets of parallaxes. The mean and/or median Hipparcos parallax is equal to the mean and/or median secular parallax (either Hipparcos or Tycho-2) to within $\lesssim 0.10$ mas. This implies that the secular parallaxes are reliable and do not suffer from a significant systematic component (cf. Sect. 6). This conclusion is supported by Table 3, which compares trigonometric and secular parallaxes for three Hyades binaries which also have orbital parallaxes.

The goodness-of-fit parameter $g_{\text {Hip }}$ allows a natural division between high-fidelity kinematic members $\left(g_{\text {Hip }} \leq 9\right)$ and kinematically deviant stars $\left(g_{\text {Hip }}>9\right.$; Sect. 2.2 ; cf. Fig. 5). The latter are not necessarily non-members, but can also be (close) multiple stars for which the Hipparcos proper motions do not properly reflect the center-of-mass motion (Sect. 4.3). Fifty of the 197 P98 members with known radial velocities have $g_{\text {Hip }}>9$, which leaves a number of high-fidelity members similar to that found by Dravins et al. (1997; 133 stars), Madsen (1999; 136 stars), and Narayanan \& Gould (1999b; 132 stars) (cf. Table 3 in Lindegren et al. 2000). Fourteen of the 21 possible P98 members (Col. $(\mathrm{x})=$ "?" in their Table 2) have $g_{\text {Hip }}>9$. These stars do not have measured radial velocities (Sect. 4.1), and P98 membership is based on proper motion data only. All but one of these stars are rejected as Hyades members by de Bruijne (1999a) and/or Hoogerwerf \& Aguilar (1999; Table A.1; cf. Sect. 4.2). The 14 suspect secular parallaxes thus most likely indicate these objects are non-members.

\subsection{Hipparcos: Additional candidates}

Table A.2 lists secular parallaxes for the 15 additional candidate members (Sect. 4.2). The assumption that these stars share the same space motion as the Hyades cluster (in other words: the assumption of membership) generally results in both high values for the goodness-of-fit parameters $g_{\text {Hip }}$ and secular parallaxes which are inconsistent with the trigonometric values. This means these stars are 
Table 3. Hipparcos and Tycho-2 secular parallaxes, and associated goodness-of-fit parameters $g_{\text {Hip }}$ and $g_{\text {Tycho-2 }}$, for the binaries which have both trigonometric (ESA 1997) and orbital parallaxes (Torres et al. 1997a,b,c)

\begin{tabular}{|c|c|c|c|c|c|c|c|}
\hline HIP & TYC & $\begin{array}{l}\pi_{\text {Hip }} \\
\text { mas }\end{array}$ & $\begin{array}{c}\pi_{\mathrm{sec}, \mathrm{Hip}} \\
\text { mas }\end{array}$ & $\begin{array}{c}\pi_{\mathrm{sec}, \text { Tycho }-2} \\
\text { mas }\end{array}$ & $\begin{array}{l}\pi_{\text {orb }} \\
\text { mas }\end{array}$ & $g_{\text {Hip }}$ & $g_{\text {Tycho }-2}$ \\
\hline 20087 & $\begin{array}{llll}1276 & 1622 & 1\end{array}$ & $18.25 \pm 0.82$ & $18.31 \pm 0.69$ & $18.70 \pm 0.29$ & $17.92 \pm 0.58$ & 0.19 & 0.00 \\
\hline $20661^{\mathrm{b}}$ & 126511711 & $21.47 \pm 0.97$ & $21.29 \pm 0.37$ & $21.08 \pm 0$ & $21.44 \pm 0.67$ & 7.2 & 0.00 \\
\hline $20894^{\mathrm{C}}$ & 126511721 & $21.89 \pm 0.83$ & $22.24 \pm 0.36$ & $22.35 \pm 0.36$ & $21.22 \pm 0.76$ & 0.26 & 0.22 \\
\hline
\end{tabular}

${ }^{\mathrm{a}}: \pi_{\text {trigonometric }}=19.4 \pm 1.1$ mas $\left(\right.$ Gatewood et al. 1992) and $\pi_{\text {trigonometric }}=18.23 \pm 0.86$ mas (Söderhjelm 1999).

${ }^{\mathrm{b}}: \pi_{\text {trigonometric }}=22.1 \pm 1.1$ mas (Söderhjelm 1999).

c: See Sects. 10.3 and B.3 for a discussion of this system.

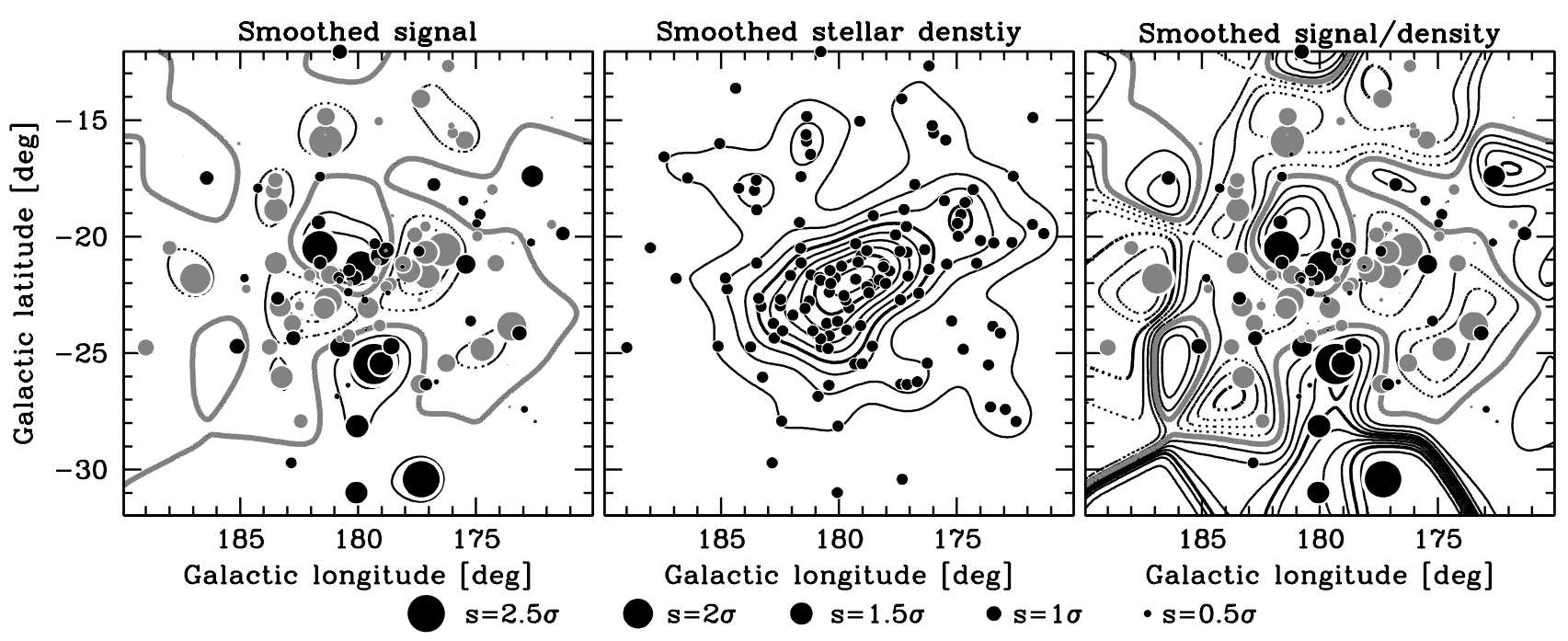

Fig. 4. Left: the trigonometric-minus-secular parallax difference field $s(\ell, b)$ (Eq. (4)), smoothed using the Gaussian kernel $G(\ell, b)$ (Eq. (5)) with smoothing length $\sigma_{\mathrm{s}}=1^{\circ}$. Solid contours correspond to $s \geq 0$; dotted contours correspond to $s<0$; gray contours denote $s=0$. Heavy/light contours are spaced by 1.0/0.25. The dots indicate the positions of 127 Hyades with non-suspect secular parallaxes $\left(g_{\text {Hip }} \leq 9\right)$. Black symbols have $s \geq 0$ (60 stars), while gray symbols have $s<0$ (67 stars). The symbol sizes correspond linearly to the strength of the signal $s$ (larger symbols denote larger $|s|$; see legend). Middle: as left panel, but for the projected stellar number density $\rho(\ell, b) \equiv \delta_{\mathrm{D}}(\ell, b)$. The lowest contour level equals $0.25 \mathrm{star}^{\mathrm{deg}}{ }^{-2}$. Right: as left panel, but for the signal $s(\ell, b)$ divided by the density $\rho(\ell, b)$

likely non-members (cf. Sect. 4.2 in B99b); only three of them (HIP 19757, 21760, and 25730) have $g_{\text {Hip }} \leq 9$. In retrospect, especially HIP 19757 is a likely new member: it was selected as candidate both by de Bruijne (1999a) and Hoogerwerf \& Aguilar (1999); it has an uncertain trigonometric parallax $\left(\pi_{\text {Hip }}=16.56 \pm 4.48\right.$ mas $)$ due to its faint magnitude $(V=11.09 \mathrm{mag})$; it has a Hipparcos secular parallax $\left(\pi_{\text {sec } \text { Hip }}=20.19 \pm 1.04\right.$ mas; $\left.g_{\text {Hip }}=2.67\right)$ which places it at only $7.15 \mathrm{pc}$ from the cluster center; its Hipparcos secular parallax puts it on the Hyades main sequence (Sect. 8); and it has an unknown radial velocity.

\subsection{Tycho-2: Faint candidates}

The "Base de Données des Amas ouverts" database (BDA; http://obswww. unige.ch/webda/webda.html) contains 23 photometric Hyades which are not contained in the Hipparcos Catalogue but which were observed by Tycho (cf. Sect. 3.1 and Fig. 2 in P98). The Tycho-2 secular parallaxes of most of these stars lie between 18 and 22 mas, indicating they are located at the same distance as the bulk of the bright Hyades. Only four of them have $g_{\text {Tycho-2 }}>9$. Most of these stars are thus likely members. We discuss their HR diagram positions in Sect. 8 .

\subsection{Random secular parallax errors}

Table A.1 contains random secular parallax errors resulting from both uncertainties in the underlying proper motions and the internal velocity dispersion in the cluster $\left(\sigma_{v}=0.30 \mathrm{~km} \mathrm{~s}^{-1}\right.$; Sect. 4.1 in B99b). Hipparcos/Tycho-2 secular parallax errors for Hyades are on average a factor $\sim 3.0$ smaller than the corresponding Hipparcos trigonometric parallax errors.

\section{Systematic secular parallax errors?}

Although the secular parallaxes derived in Sect. 5 have small random errors, they might suffer from significant systematic errors. In this section, we investigate the 
influences of the maximum likelihood method, the uncertainty of the tangential component of the cluster space motion (Sect. 6.1), the correlated Hipparcos measurements (Sect. 6.2), as well as possible unmodelled patterns in the velocity field of the Hyades (Sect. 6.4). Section 6.5 summarizes our results.

\subsection{Cluster space motion}

Extensive Monte Carlo tests of the maximum likelihood procedure (Sects. 2.2-2.3) show that, given the correct cluster space motion, the method is not expected to yield systematic secular parallax errors larger than a few hundredths of a mas (e.g., Sects. 3.2.2.1 and 3.2.2.3, Table 3, and Fig. 5 in B99b). It is possible, though, that a systematic error is introduced through the use of an incorrect value for the tangential component of the cluster space motion $\left(v_{\|}\right.$; Sect. 3.2). We estimate $\sigma_{v_{\|}} \sim 0.15 \mathrm{~km} \mathrm{~s}^{-1}$ from Table 1 . This uncertainty gives rise to maximum systematic secular parallax errors of $\sim 0.14$ mas $\left(\sigma_{v_{\|}}=\right.$ $0.30 \mathrm{~km} \mathrm{~s}^{-1}$ gives 0.28 mas; see Sect. 4.2 in B99b and use $v_{\|}=24.35 \mathrm{~km} \mathrm{~s}^{-1}, \bar{\mu}=111.0$ mas $\mathrm{yr}^{-1}, \sigma_{\bar{\mu}}=$

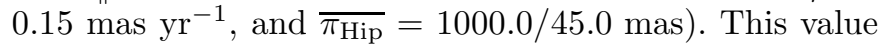
compares favorably to typical random secular parallax errors for Hyades ( $\sim .50$ mas; Table A.1). It is, nonetheless, desirable to obtain a more precise estimate of the tangential component of the cluster space motion. This requires a better knowledge of the associated radial space motion component and internal velocity dispersion and/or more precise proper motion measurements (Sect. 11).

\subsection{Hipparcos correlations on small angular scales}

The Hipparcos Catalogue contains absolute astrometric data. Absolute in this sense should be interpreted as lacking global systematic errors at the $\sim 0.10$ mas $\left(\mathrm{yr}^{-1}\right)$ level or larger (ESA 1997; cf. Narayanan \& Gould 1999a, who quote an upper limit of 0.47 mas for the Hyades field). However, the measurement principle of the satellite does allow for the existence of correlated astrometric parameters on small angular scales $\left(\sim 1^{\circ}-3^{\circ}\right.$; e.g., Lindegren 1989 ; ESA 1997, vol. 3, pp. 323 and 369). These correlations have been suggested to be responsible for the so-called "Pleiades anomaly", i.e., the fact that the mean distance of the Pleiades cluster as derived from the mean Hipparcos trigonometric parallax differs from the value derived from stellar evolutionary modelling (Pinsonneault et al. 1998; but see, e.g., Robichon et al. 1999; van Leeuwen 1999).

The left panel of Fig. 4 shows the $1^{\circ}$-smoothed errornormalized difference field of the Hipparcos trigonometric minus secular parallaxes for all stars with non-suspect secular parallaxes $\left(g_{\text {Hip }} \leq 9\right)$ in the center of the Hyades cluster $\left(1700^{\circ} \leq \ell \leq 190^{\circ} .0\right.$ and $-32^{\circ} .0 \leq b \leq-12.0 ; \ell$ and $b$ denote Galactic coordinates). In order to obtain this field, we convolved the sum of the discrete quantity $s$ :

$s=s(\ell, b) \equiv \delta_{\mathrm{D}}(\ell, b) \cdot \frac{\pi_{\mathrm{Hip}}-\pi_{\mathrm{sec}, \mathrm{Hip}}}{\sqrt{\left(\sigma_{\pi, \mathrm{Hip}}^{2}+\sigma_{\pi, \mathrm{sec}, \mathrm{Hip}}^{2}\right)}}$ where $\delta_{\mathrm{D}}$ denotes the two-dimensional Dirac delta function, for each star with the normalized two-dimensional Gaussian smoothing kernel

$G(\ell, b) \equiv \frac{1}{2 \pi \sigma_{\mathrm{s}}^{2}} \cdot \exp \left(-\frac{1}{2}\left[\frac{\ell^{2}+b^{2}}{\sigma_{\mathrm{s}}^{2}}\right]\right)$,

where $\sigma_{\mathrm{s}}=1^{\circ}$ is the smoothing length. The appearance of the difference field depends on the adopted smoothing length, though not very sensitively. Taking a large smoothing length returns a smooth field, whereas a small smoothing length gives a "spiky distribution", reminiscent of the original delta function-type field (Eq. (4)). Given a Hyad, its closest neighbour on the sky is typically found at an angular separation of $\sim 0.5$. Our choice of the smoothing length (1.0) corresponds to the median value (for all stars) of the median angular separation of the $\sim 3-4$ nearest neighbours on the sky. We checked that the smoothed difference field has the same overall appearance when adopting smoothing lengths of 0.5 or $2^{\circ} .0$.

The smoothed difference field shows several positive and negative peaks with a full-width-at-half-maximum of a few degrees. These peaks can be due to spatially correlated errors in the Hipparcos parallaxes $\pi_{\text {Hip }}$ on small angular scales, spatially correlated errors in the Hipparcos secular parallaxes $\pi_{\text {sec,Hip }}$ on small angular scales, or both. From the fact that the peaks are not evident in the smoothed difference field of the Hipparcos secular parallaxes and the mean cluster parallax (not shown), whereas they are present in the smoothed difference field of the Hipparcos trigonometric parallaxes and the mean parallax (not shown), we conclude that they are mainly caused by correlated Hipparcos measurements, notably the trigonometric parallaxes (cf. Narayanan \& Gould 1999b). As the relative precision of the Hipparcos proper motions is $\sim 5$ times higher than the relative precision of the Hipparcos trigonometric parallaxes (cf. Sect. 1), the Hipparcos secular parallaxes, which have more-or-less been directly derived from the Hipparcos proper motions, are correlated as well, though with smaller "amplitudes".

The quantity $s$ (Eq. (4)) denotes, for a given star, the dimensionless significance (in terms of the effective Gaussian standard deviation $\sigma \equiv\left(\sigma_{\pi, \mathrm{Hip}}^{2}+\sigma_{\pi, \mathrm{sec}, \mathrm{Hip}}^{2}\right)^{1 / 2} \sim$ 1.5 mas) of the parallax difference $\pi_{\text {Hip }}-\pi_{\text {sec,Hip. As the }}$ smoothing kernel $G$ (Eq. (5)) is properly normalized to unit area in two dimensions, the smoothed difference field can be interpreted in terms of net significance per square degree. The right panel of Fig. 4 shows the corresponding smoothed difference field expressed in terms of net significance per star. This field was obtained by dividing the smoothed signal field by an identically smoothed stellar number density field $\rho(\ell, b) \equiv \delta_{\mathrm{D}}(\ell, b)$ (middle panel of Fig. 4). The smoothed difference field expressing significance per star also contains patches of size a few degrees with both negative and positive contributions, although there is no large-scale trend (cf. Fig. 3). We thus conclude that the Hipparcos trigonometric parallaxes towards the Hyades cluster are spatially correlated over angular scales 


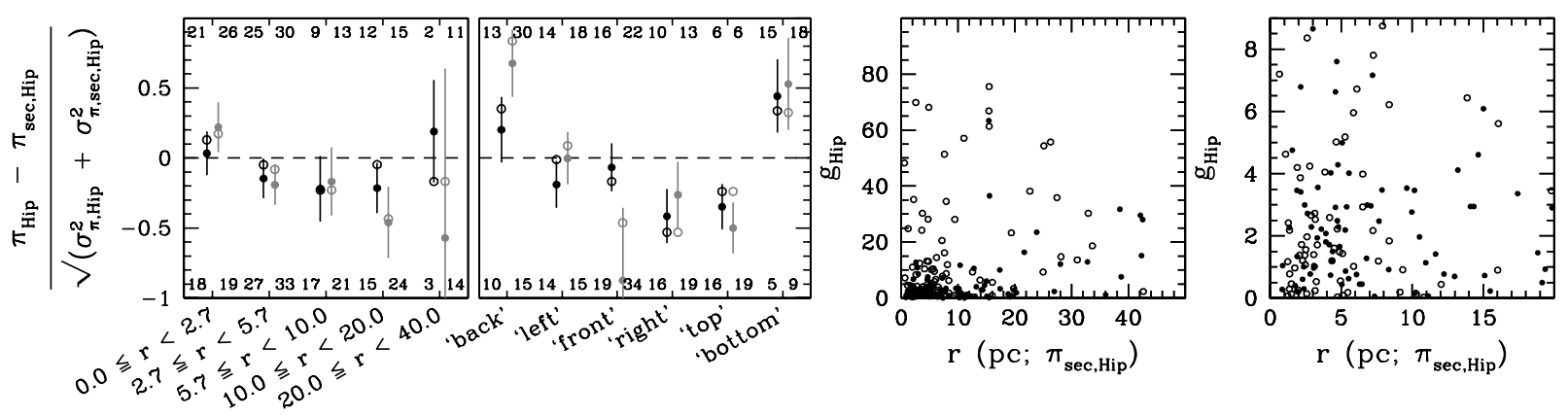

Fig. 5. Left two panels: normalized difference between Hipparcos trigonometric and secular parallaxes for the 218 P 98 members as function of location in the cluster. The left panel shows a spatial division according to $r$ (the three-dimensional distance to the cluster center using Hipparcos secular parallaxes) in five spherical annuli (from center to core radius [2.7 pc] to half-mass radius $[5.7 \mathrm{pc}]$ to tidal radius $[10 \mathrm{pc}]$ to two tidal radii $[20 \mathrm{pc}]$ to $40 \mathrm{pc})$. The cluster center is $\left(b_{u}, b_{v}, b_{w}\right)=(-43.37,0.40,-17.46) \mathrm{pc}$ in Galactic Cartesian coordinates (cf. Table 3 in P98). The right panel shows a spatial division according to the six equal-volume pyramids (Sect. 6.3; orientation in Galactic coordinates). Black/gray symbols show results for stars with $g_{\mathrm{Hip}} \leq 9 / \infty$. Filled symbols and vertical lines denote mean and standard deviation ( $\pm 1 \sigma$ uncertainty), while open symbols denote median values; the numbers in the upper/lower halves of the panels denote the corresponding numbers of stars with $\pi_{\mathrm{Hip}}-\pi_{\mathrm{sec}, \mathrm{Hip}}>/ \leq 0$. Right two panels: the goodness-of-fit parameter $g_{\mathrm{Hip}}$ as function of $r$ (left; 202 stars) and a magnification of this panel for 144 high-fidelity (i.e., low- $g_{\text {Hip }}$ ) members in the central parts of the cluster (right). Open symbols denote multiple stars (i.e., either Col. (s) in P98's Table 2 is "SB" or "RV", Col. (t) is "H", "I", or "M", or Col. (u) is one of "C G O V X S"; 103/64 entries in the left/right panel)

of a few degrees. The maximum deviations in the central region of the cluster $\left(\rho \gtrsim 0.5 \mathrm{star}^{\mathrm{deg}}{ }^{-2}\right)$, however, are generally less than $\sim 0.50-0.75 \sigma$ per star (i.e., $\lesssim 0.75$ $1.00 \mathrm{mas})$. The signal in the outer parts of the cluster is statistically non-interpretable as it is severely influenced by the contributions of individual stars.

Our conclusions are qualitatively consistent with the results of Narayanan \& Gould (1999b, their Fig. 9 and Sect. 6.2; cf. Lindegren et al. 2000). These authors, however, overestimate, by a factor of $\sim 2$, the strength of the correlation by claiming that "the Hipparcos parallaxes toward the Hyades are spatially correlated over angular scales of a few degrees, with an amplitude of about 12 mas".

\subsection{Three-dimensional location within the cluster}

Figure 3 (Sect. 5) shows that the secular parallaxes as a set (i.e., averaged over all regions within the cluster) are statistically identical to the Hipparcos trigonometric parallaxes. Figure 5 compares Hipparcos secular and trigonometric parallaxes as function of the threedimensional distance $r$ to the cluster center and as function of spatial location within the cluster according to an equal-volume pyramid division: we divide (an artificial) three-dimensional box containing all cluster members in six adjacent equal-volume pyramids, all of them having their top at the cluster center. This division, as viewed from the Sun in Galactic coordinates, yields six distinct regions: "back", "left" (i.e., towards smaller longitudes), "front", "right" (i.e., towards larger longitudes), "top" (i.e., towards larger latitudes), and "bottom" (i.e., towards smaller latitudes). Although systematic differences seem to be present in Fig. 5, they are smaller than a few tenths of the median effective parallax uncertainty $\left(\sigma_{\pi, \text { Hip }}{ }^{2}+\sigma_{\pi, \text { sec }, \text { Hip }}{ }^{2}\right)^{1 / 2} \sim 1.0-1.5$ mas. Figure 5 also shows that the distribution of the goodness-of-fit parameter $g_{\text {Hip }}$ does not show unwarranted dependencies on distance from the cluster center. We thus conclude that secular parallaxes for stars in the inner and outer regions of the cluster do not differ significantly (i.e., at the $\sim 0.30$ mas level or larger).

\subsection{Cluster velocity field}

The method described in Sects. 2.2-2.3 assumes that the expectation values $E\left(\mathbf{v}_{i}\right)$ of the individual stellar velocities $\mathbf{v}_{i}(i=1, \ldots, n)$ equal the cluster space motion $\mathbf{v}$ (cf. Sect. 3). A random internal velocity dispersion in addition to this common space motion is allowed and accounted for in the procedure, as random motions do not affect $E\left(\mathbf{v}_{i}\right)$ by definition. However, a systematic velocity pattern, such as expansion or contraction, rotation, and shear, has not been taken into account in the modelling. The application of the procedure to data subject to velocity patterns is thus bound to lead to incorrect and/or biased results.

\subsubsection{Pre-Hipparcos results}

Many studies have been devoted to the detection or exclusion of velocity structure in the Hyades (see P98 for an overview), although $N$-body simulations of open clusters generally predict that, for gravitationally bound groups like the Hyades, velocity patterns are generally too small to be measured with present-day data (e.g., Dravins et al. 1997; Sect. 7.2 in P98). In view of the Hyades age $(\tau=$ $625 \pm 50$ Myr; P98), shear is not likely to be present in the core and corona: using $\sigma_{v}=0.30 \mathrm{~km} \mathrm{~s}^{-1}$ and a half-mass radius of 5 pc (Pels et al. 1975), it follows that 

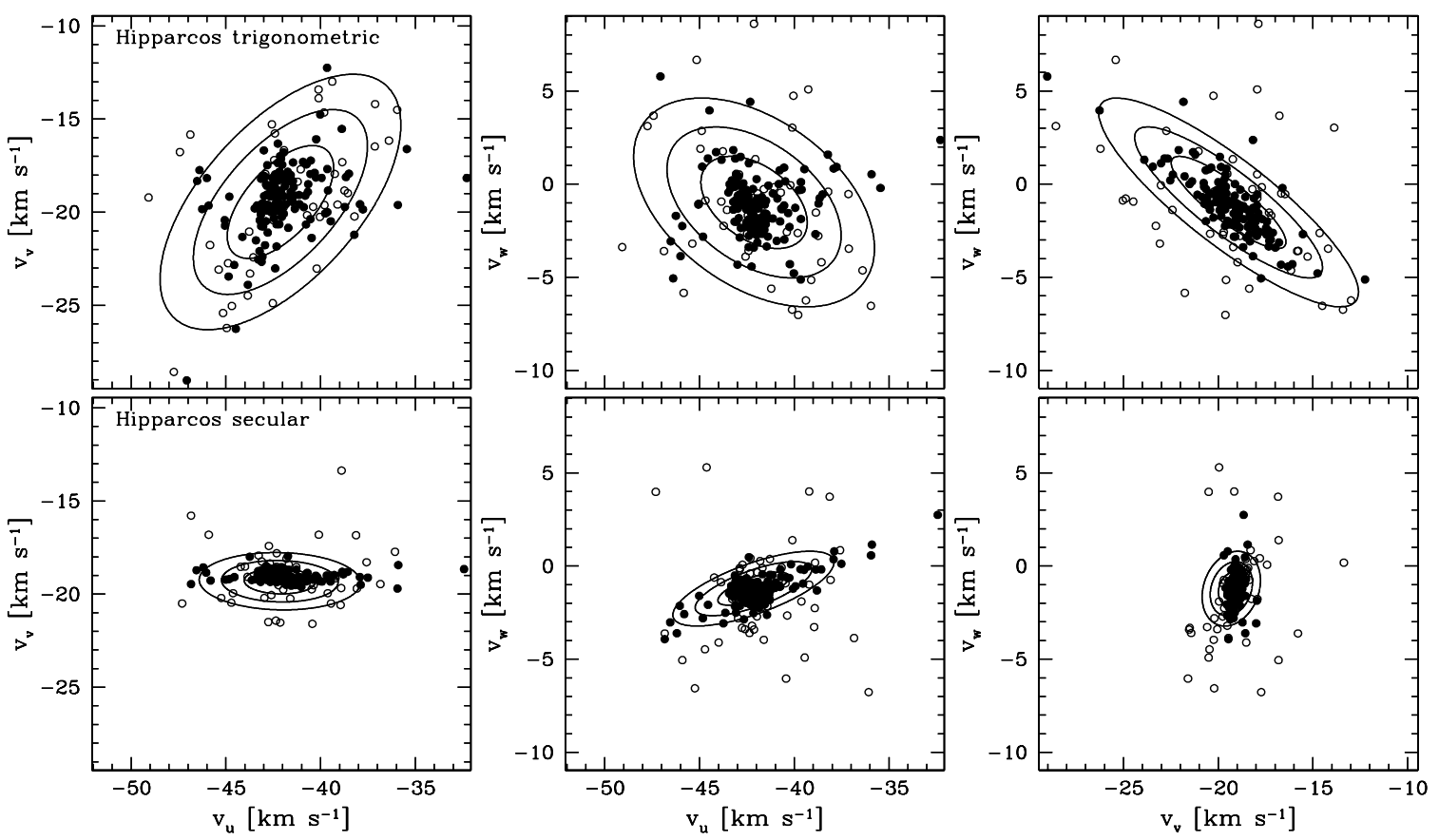

Fig. 6. Top row: three-dimensional velocity distribution in Galactic Cartesian coordinates $\left(v_{u}, v_{v}, v_{w}\right)$, based on Hipparcos trigonometric parallaxes, for the 197 P98 members with known radial velocities (cf. Fig. 16 in P98; symbol coding as in bottom row). The contours are centered on the (arithmetic) mean motion of all stars $(-42.07,-19.45,-0.96) \mathrm{km} \mathrm{s}^{-1}$ (cf. Table 3 in P98); they show the 1,2 , and $3 \sigma$ (i.e., $68.3,95.4$, and 99.73 per cent) confidence limits of the mean covariance matrix associated with the mean space motion (Sect. 6.4.2). All "outliers" have space motions (based on Hipparcos proper motions and trigonometric parallaxes) which are consistent with the mean motion of the cluster. Bottom row: as top row, but using Hipparcos secular parallaxes (mean motion $\left.(-42.15,-19.31,-1.21) \mathrm{km} \mathrm{s}^{-1}\right)$. Open symbols denote suspect secular parallaxes $\left(g_{\mathrm{Hip}}>9 ; 50 \mathrm{stars}\right)$

$\tau \sim 20$ crossing times, which means that the central parts of the cluster are relaxed (cf. Hanson 1975). The Hyades age also puts a rough upper limit on the linear expansion ${ }^{7}$ coefficient $K \lesssim 0.0016 \mathrm{~km} \mathrm{~s}^{-1} \mathrm{pc}^{-1}$ (resulting in a bias in the radial component of the maximum likelihood cluster space motion of $\lesssim-0.07 \mathrm{~km} \mathrm{~s}^{-1}$ ). A global rotation of the cluster could be present, although Wayman (1967; cf. Wayman et al. 1965; Hanson 1975) claimed that the cluster rotation about three mutually perpendicular axes is consistent with zero to within $0.05 \mathrm{~km} \mathrm{~s}^{-1} \mathrm{pc}^{-1}$; Gunn et al. (1988) present (weak) evidence for rotation at the level of $\lesssim 1 \mathrm{~km} \mathrm{~s}^{-1}$ radian $^{-1}$ (cf. O'Connor 1914).

\subsubsection{Hipparcos parallaxes: Perryman et al. (1998)}

Figure $8 \mathrm{~b}$ in P98 displays the three-dimensional velocity distribution $\left(v_{u}, v_{v}, v_{w}\right)$ of the 197 P98 members with known radial velocities. Although the velocity residuals seem to show evidence for shear and/or rotation, notably

\footnotetext{
7 The only astrometrically non-observable velocity pattern is an isotropic expansion at a rate $K$ (Appendix A in Dravins et al. 1999): such velocity structure cannot be disentangled from a bulk motion in the radial direction based on proper motion data only ( $K$ is the linear expansion coefficient in $\mathrm{km} \mathrm{s}^{-1} \mathrm{pc}^{-1}$; cf. Sects. 3.2.3-3.2.4 in B99b). Neglecting a uniform expansion for a cluster at a distance $D$ [pc] yields a bias in its mean radial velocity of $-D \cdot K\left[\mathrm{~km} \mathrm{~s}^{-1}\right]$ (e.g., Eq. (13) in B99b).
}

for stars in the outer regions (Fig. 9 in P98), the systematic pattern can be explained by a combination of the transformation of the observables $\left(\pi_{\text {Hip }}, \mu_{\alpha^{*}}, \mu_{\delta}, v_{\text {rad,obs }}\right)$ to the linear velocity components $\left(v_{u}, v_{v}, v_{w}\right)$ and the presence of Hipparcos data covariances: P98 show that the assumption of a common space motion for all members with a one-dimensional internal velocity dispersion of $0.30 \mathrm{~km} \mathrm{~s}^{-1}$, which allows averaging of the individual motions and associated covariance matrices for all stars, translates into a mean motion and associated mean covariance matrix (i.e., 1, 2, and $3 \sigma$ confidence regions) which adequately follow the observed velocity residuals (Sect. 7.2 and Figs. 16-17 in P98; cf. top row of Fig. 6). Therefore, P98 conclude that the observed kinematic data of the Hyades cluster is consistent with a common space motion plus a $0.30 \mathrm{~km} \mathrm{~s}^{-1}$ velocity dispersion, without the need to invoke the presence of rotation, expansion, or shear.

The motions of members beyond the tidal radius ( $\sim 10 \mathrm{pc}$ ), as opposed to the motions of gravitationally bound members in the central parts of the cluster, are predominantly influenced by the Galactic tidal field (e.g., Pels et al. 1975). These (evaporated) stars do therefore not necessarily adhere to the strict pattern of a common space motion which is present in the core and corona. The systematic velocity distortions are hard to predict, analytically and numerically, as they depend critically on the details of the evaporation mechanism(s) (e.g., Terlevich 1987). They are hard to observe as well due to both the 

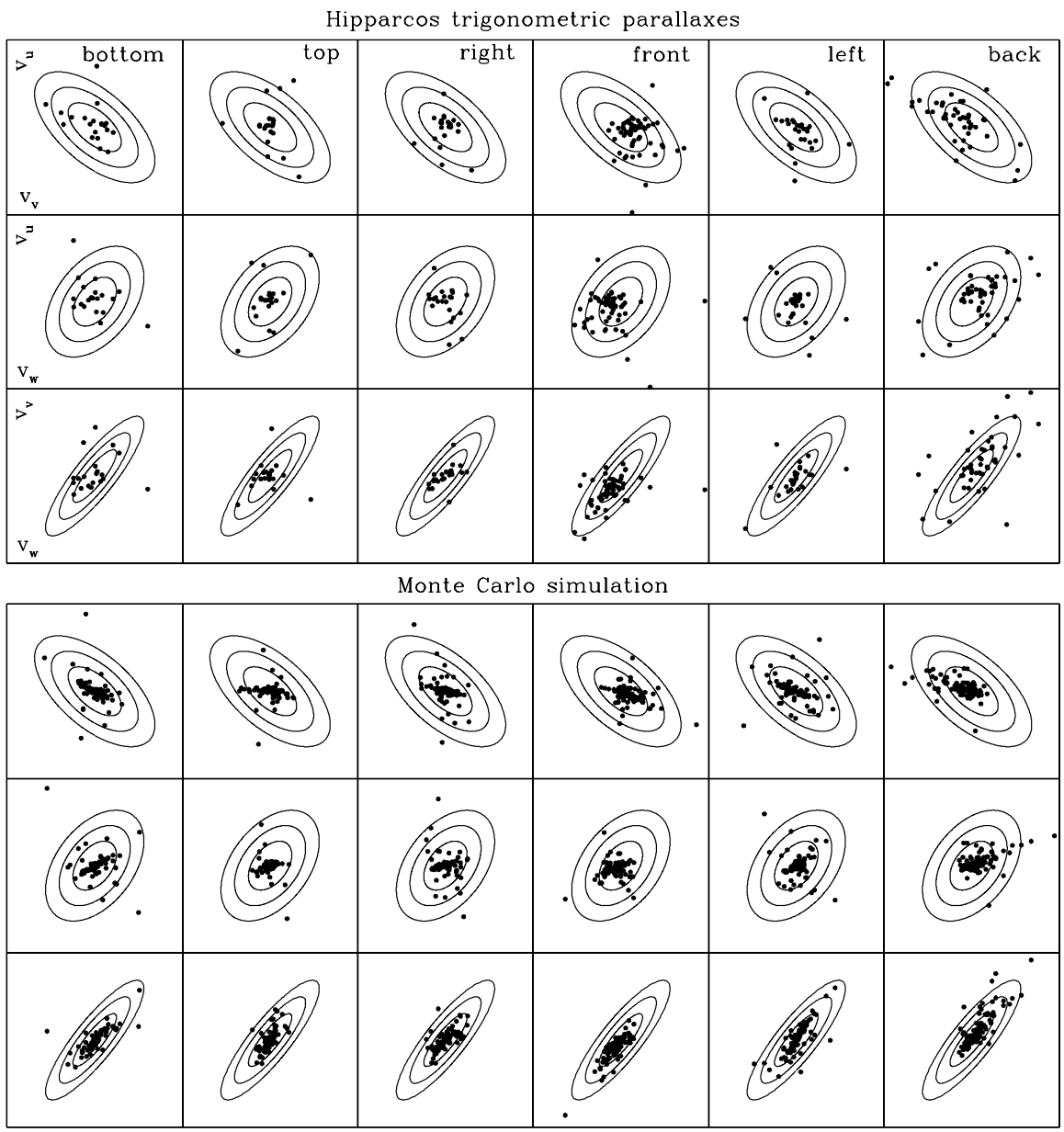

Fig. 7. Top three rows: Hipparcos trigonometric parallax-based velocity field decomposition, with respect to the mean velocity $\left(v_{u}, v_{v}, v_{w}\right)=(-42.07,-19.45,-0.96) \mathrm{km} \mathrm{s}^{-1}$ in $20 \mathrm{~km} \mathrm{~s}^{-1} \times 20 \mathrm{~km} \mathrm{~s}^{-1} \times 20 \mathrm{~km} \mathrm{~s}^{-1}$ boxes, for the 197 P98 members with known radial velocities according to the location of the stars within the cluster (from the left column right: "bottom" (21 stars), "top" (21 stars), "right" (22 stars), "front" (59 stars), "left" (28 stars), and "back" (46 stars; Sect. 6.3) and from the top down: $v_{u}$ versus $v_{v}, v_{u}$ versus $v_{w}$, and $v_{v}$ versus $v_{w}$; velocity components in a Galactic Cartesian coordinate frame). The ellipses denote 1,2 , and $3 \sigma$ confidence regions of the mean motion and associated covariance matrix (Sect. 6.4.2). The bottom series of panels are similar to the top series, but show 500 Monte Carlo stars which share a common space motion exclusively (Sect. 6.4.3)

sparse sampling of "members" and the uncertain criteria for membership in the outer regions of the cluster.

\subsubsection{Hipparcos parallaxes: This study}

Figure 7 (top series of panels) shows the Hyades velocity field, based on Hipparcos trigonometric parallaxes, for different spatial regions of the cluster (Sect. 6.3). The observed velocities are not identically distributed in each region but show systematic effects, although these are restricted to "the $3 \sigma$ confidence regions". Notably the "front" and "back" of the cluster show differences, indicative of a coupling between position and velocity, i.e., a velocity pattern. Explanations for this trend include (1) a rotation of the cluster, (2) a shearing pattern with respect to an axis, and (3) a correlation between the trigonometric parallaxes $\pi_{\text {Hip }}$ and their associated errors $\sigma_{\pi \text {,Hip }}$ (cf. Sect. 7.2 in P98).
(1) Rotation: Given the observed velocity field, we determine the Galactic coordinates of the best-fitting rotation axis $\left(\ell_{\text {rot }}\right.$ and $\left.b_{\text {rot }}\right)$ and the corresponding rotation period $\left(P_{\text {rot }}\right)$ by minimizing the dispersion of the velocity residuals with respect to the rotation axis, after adding a rotation pattern to the mean space motion. This results in the estimates $\ell_{\text {rot }} \sim 131.5, b_{\text {rot }} \sim+60$. 0 , and $P_{\text {rot }}=68.0 \mathrm{Myr}$ (i.e., $\sim 0.10 \mathrm{~km} \mathrm{~s}^{-1} \mathrm{pc}^{-1}$ ).

(2) Shear: A shear pattern with respect to an axis pointing towards $\ell_{\text {shear }}$ and $b_{\text {shear }}$ is described by a constant $P_{\text {shear }}$ which expresses the strength of the shear. A least-squares fit returns $\ell_{\text {shear }} \sim 131.5, b_{\text {shear }} \sim+60^{\circ} 0$, and $P_{\text {shear }} \sim$ $0.13 \mathrm{~km} \mathrm{~s}^{-1} \mathrm{pc}^{-1}$ (as our proper motion, parallax, and radial velocity data do not have enough discriminating power to reveal the subtle differences between a rotation and shear pattern, our fit returns a shear axis which is identical to the rotation axis listed above). 

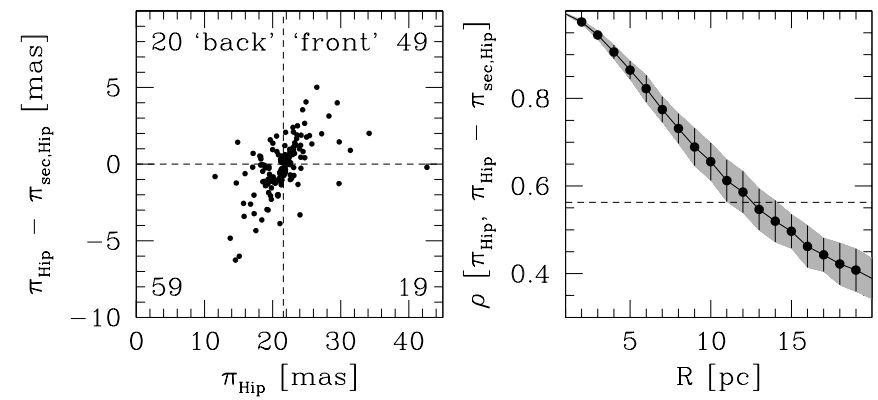

Fig. 8. Left: an estimate of the Hipparcos trigonometric parallax error $\sigma_{\pi \text {,Hip }}\left(\pi_{\text {Hip }}-\pi_{\text {sec } \text { Hip }} \approx \pi_{\text {Hip }}-\pi_{\text {true }} \equiv \Delta_{\pi, \text { Hip }}\right)$ versus the Hipparcos trigonometric parallax $\pi_{\text {Hip }}$ for the 147 P98 Hyades members with non-suspect secular parallaxes $\left(g_{\text {Hip }} \leq\right.$ 9 ). Every sample of stars with (nearly) equal true parallaxes shows a correlation between $\Delta_{\pi, \mathrm{Hip}}\left(\right.$ or: $\sigma_{\pi, \mathrm{Hip}}$ ) and $\pi_{\mathrm{Hip}}$; we find a correlation coefficient $\rho=+0.56$. The dashed vertical line denotes the mean distance of the cluster $(D=46.34 \mathrm{pc}$; $\pi=21.58$ mas; Table 3 in P98). The numbers in the corners of the four quadrants denote the numbers of stars in the corresponding regions. Right: the mean correlation coefficient for 100 Monte Carlo realizations of a Hyades-like cluster as function of the cluster radius $R$. Each cluster has a homogeneous number density. The dots and vertical lines denote the mean value of $\rho$ and the corresponding standard deviation; the gray band denotes the $\pm 1 \sigma$ region. The dashed horizontal line indicates the observed value $\rho=+0.56$

(3) Correlated trigonometric parallaxes and errors: The lower series of panels in Fig. 7 show a velocity field decomposition for a realistic Monte Carlo realization of the Hyades (500 stars, 10 pc radius, including Hipparcos data covariances) in which the stars share a common space motion exclusively. Despite the absence of intrinsic velocity structure, the "front" and "back" distributions do show a systematic pattern which resembles the observed distribution (upper series of panels) remarkably well. P98 (their Sect. 7.2) did already argue that correlated velocity residuals are a natural result of the presence of a correlation between the Hipparcos parallaxes $\pi_{\text {Hip }}$ and the corresponding observational errors $\sigma_{\pi \text {,Hip }}$ in a sample of Hyades members (left panel of Fig. 8; we find a correlation coefficient $\rho=+0.56$ between $\pi_{\text {Hip }}-\pi_{\text {sec,Hip }} \approx$ $\pi_{\text {Hip }}-\pi_{\text {true }} \equiv \Delta_{\pi \text {,Hip }}$ and $\left.\pi_{\text {Hip }}\right)$. Although the individual Hipparcos trigonometric parallaxes are not correlated with their associated observational errors, the selection of a set of stars with (nearly) equal true parallaxes, such as the members of an open cluster, induces the presence of a correlation in the sample: Hyades with large observed parallaxes are, in general, more likely to have $\Delta_{\pi, \text { Hip }} \equiv \pi_{\text {Hip }}-\pi_{\text {true }}>0$ than $\sigma_{\pi \text {,Hip }}<0$ (and vice versa for Hyades with small observed parallaxes). The strength of this correlation between the (sign of the) parallax error and the observed parallax depends on the intrinsic size of the cluster: a small cluster gives a small spread in true parallaxes, which implies a large correlation. The right panel of Fig. 8 shows the mean correlation coefficient derived from Monte Carlo realizations of a Hyades-like cluster as function of the cluster radius $R$. The observed correlation coefficient $\rho=+0.56$ implies $R \sim 10-15 \mathrm{pc} \sim 1.0-1.5 r_{\mathrm{t}}$, which is a very reasonable definition for the size of the Hyades cluster.

Discussion: The analysis presented above shows that the systematic velocity pattern displayed in Fig. 7 can be due to rotation, shear, and/or a correlation between $\pi_{\text {Hip }}$ and $\sigma_{\pi, \text { Hip }}$. Both rotation and shear provide an equally good representation of the observations, but imply a significant systematic velocity of $\sim 1 \mathrm{~km} \mathrm{~s}^{-1}$ at the tidal radius of the cluster $\left(r_{\mathrm{t}} \sim 10 \mathrm{pc}\right)$. Unmodelled systematic velocities at the level of $1-2 \mathrm{~km} \mathrm{~s}^{-1}$ in the outer regions of the cluster $\left(r_{\mathrm{t}} \lesssim r \lesssim 2 r_{\mathrm{t}}\right)$ would lead to systematic secular parallax errors as large as 0.9-1.8 mas. These values, however, are a factor 3-6 larger than the observed upper limit of 0.3 mas at $r \sim 20$ pc (Fig. 5; Sect. 6.3), which argues against an explanation of the velocity pattern in terms of rotation or shear. There is, moreover, also a direct argument in favour of the apparent velocity pattern not being caused by rotation or shear, but by the correlation between the observed parallaxes and the parallax errors instead, for this should result in an apparent rotation or shear axis pointing towards $\left(b_{u}, b_{v}, b_{w}\right) \times\left(v_{u}, v_{v}, v_{w}\right)$, i.e., $(\ell, b)=\left(116^{\circ},+48^{\circ}\right)$ $\left(\left[b_{u}, b_{v}, b_{w}\right]\right.$ and $\left[v_{u}, v_{v}, v_{w}\right]$ denote, respectively, the position and velocity vector of the cluster center expressed in Galactic Cartesian coordinates). This axis coincides within $15^{\circ}$ with the rotation and shear axes found above. We therefore conclude that the observed correlation between the Hipparcos trigonometric parallaxes and their associated random errors (Fig. 8) is mainly responsible for the (apparent) velocity structure of the Hyades (Fig. 7; cf. P98).

\subsubsection{Secular parallaxes}

Studying the Hyades velocity field using secular parallaxes, which were derived under the assumption of a specific velocity field, is of limited scientific merit. We therefore restrict such an analysis to the straightforward comparison of the input and output velocity fields (bottom row of Fig. 6), which turn out to be fully consistent. A systematic pattern as observed in the trigonometric parallax velocity field (Sect. 6.4.3) is absent in the secular parallax velocity field (not shown).

\subsection{Summary}

Monte Carlo tests combined with the uncertainty of the tangential component of the cluster space motion set the maximum expected systematic Hipparcos secular parallax error at $\sim 0.30$ mas (Sect. 6.1). This value is consistent with the facts that (1) the secular parallaxes as a set are statistically consistent with the Hipparcos trigonometric parallaxes within $\lesssim 0.10$ mas (Fig. 3; Sect. 5), and (2) secular parallaxes for stars in the inner and outer regions of the Hyades do not differ significantly, i.e., at the $\sim 0.30$ mas 

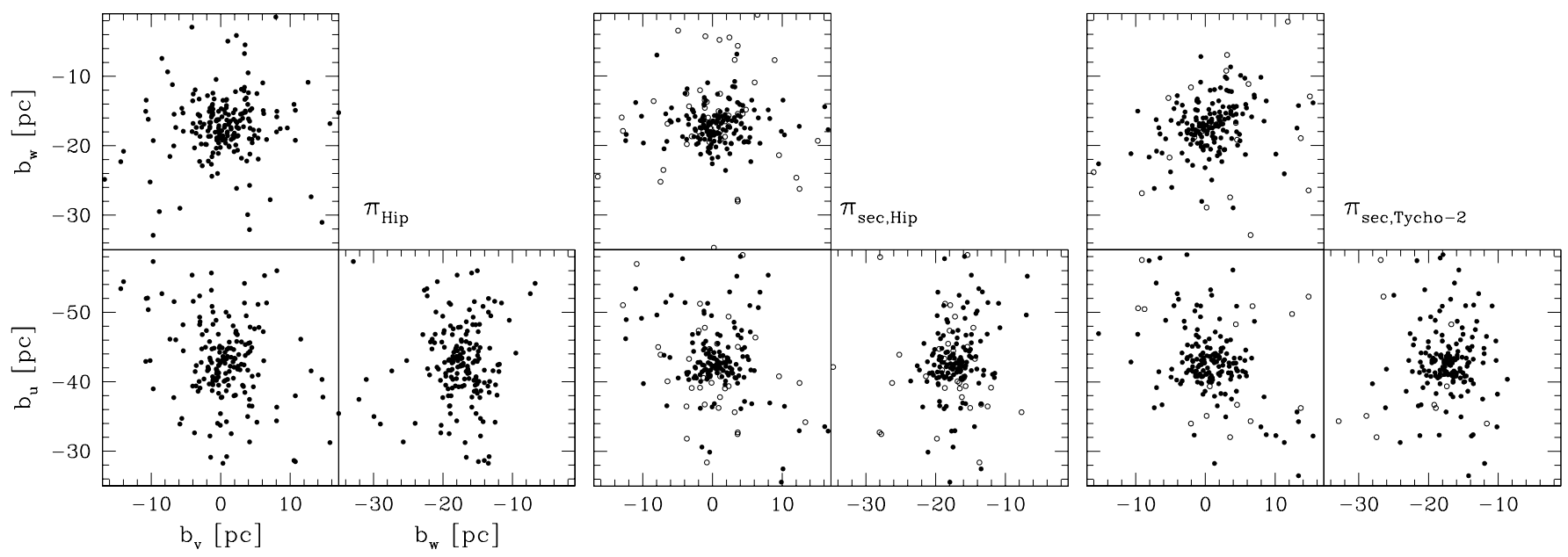

Fig. 9. Left: three-dimensional distribution, based on Hipparcos trigonometric parallaxes, of the 218 P98 members in Galactic Cartesian coordinates $\left(b_{u}, b_{v}, b_{w}\right)$ (in pc; cf. Figs. 8a-9 in P98). Some stars fall outside the plotted range. Middle: as left, but using Hipparcos secular parallaxes (154 stars with $g_{\text {Hip }} \leq 9$ [filled symbols] and 64 stars with $g_{\text {Hip }}>9$ [open symbols]). Right: as middle, but using Tycho-2 secular parallaxes (176/32 filled/open symbols)

level or larger (Fig. 5; Sect. 6.3). We conclude that secular parallaxes for Hyades within at least $r \lesssim 2 r_{\mathrm{t}} \sim 20 \mathrm{pc}$ of the cluster center can be regarded as absolute, i.e., having systematic errors smaller than $\sim 0.30$ mas.

The Hipparcos trigonometric parallax errors are correlated on angular scales of a few degrees with "amplitudes" smaller than $\sim 0.75-1.00$ mas per star (Sect. 6.2). The mean trigonometric parallax of the Hyades, however, is accurate to within $\lesssim 0.10$ mas, as regions with positive and negative contributions cancel when averaging parallaxes over the large angular extent of the cluster.

The observed lack of significant systematics in the secular parallaxes puts an upper limit on the size of possible velocity patterns (rotation or shear) of a few hundredths of a $\mathrm{km} \mathrm{s}^{-1} \mathrm{pc}^{-1}$ (Sect. 6.4.3). This upper limit, in its turn, strongly suggests that the observed systematics in the trigonometric parallax-based velocity field (Fig. 7) are due to the presence of a correlation between the Hipparcos parallaxes and their associated random errors in our sample of Hyades.

\section{Spatial structure}

At the mean distance of the Hyades, a parallax uncertainty of $\sigma_{\pi}$ (mas) corresponds to a distance error of $\sigma_{\pi} D^{2} / 1000 \sim 2 \sigma_{\pi}$ pc $(D \sim 45 \mathrm{pc})$. Typical Hipparcos parallax errors are 1.0-1.5 mas, thus yielding a $\sim 2-3 \mathrm{pc}$ distance resolution. Typical Hipparcos secular parallaxes are $\sim 3$ times more accurate than the trigonometric values (Sect. 5.4). However, because the Hipparcos resolution is already sufficient to resolve the internal structure of the Hyades (with core and tidal radii of 2.7 and $10 \mathrm{pc}$, respectively; Sect. 4.1), secular parallaxes cannot fundamentally improve upon the P98 results regarding, e.g., the three-dimensional spatial distribution of stars in the cluster, including the shape of the core and corona and flattening of the halo, "the Hyades distance" 8 , the density and mass distribution of stars in the cluster, its gravitational potential, moments of inertia, etc. (Sects. 7-8 in P98; we investigated all aforementioned examples using secular parallaxes, but were unable to obtain results which had not already been derived by P98). Figure 9, for example, shows the three-dimensional distribution of the 218 P98 members. Although the internal spatial structure of the Hyades is resolved by the Hipparcos trigonometric parallaxes, the Hipparcos secular parallaxes do provide a sharper view.

\section{Colour-absolute magnitude diagram}

The colour-absolute magnitude and HR diagrams of the Hyades cluster have been studied extensively, mainly owing to the small distance of the cluster. Among the advantages of this proximity are the negligible interstellar reddening and extinction (e.g., Crawford 1975; Taylor $1980 ; E(B-V)=0.003 \pm 0.002 \mathrm{mag})$ and the possibility to probe the cluster (main sequence) down to low masses relatively easily. As mentioned in Sect. 1, the significant cluster depth along the line of sight has always complicated pre-Hipparcos stellar evolutionary modelling (cf. Sect. 9.0 in P98). Unfortunately, even Hipparcos parallax uncertainties (typically 1.0-1.5 mas) translate into absolute magnitude errors of $\gtrsim 0.10 \mathrm{mag}$ at the mean distance of the cluster $(D \sim 45 \mathrm{pc})$, whereas $V$-band photometric errors only account for $\lesssim 0.01$ mag uncertainties for most members. The Hipparcos secular parallaxes derived in Sect. 5 are on average a factor $\sim 3$ times more precise than the Hipparcos trigonometric values (i.e., $\sigma_{\pi, \mathrm{sec}, \mathrm{Hip}} \lesssim 0.5$ mas $\sim 0.05$ mag; Sect. 5.4).

\footnotetext{
8 The statistical consistency between the Hipparcos trigonometric and secular parallaxes as a set (e.g., Fig. 3) implies that the (mean) Hyades distance derived by P98 cannot be improved upon.
} 


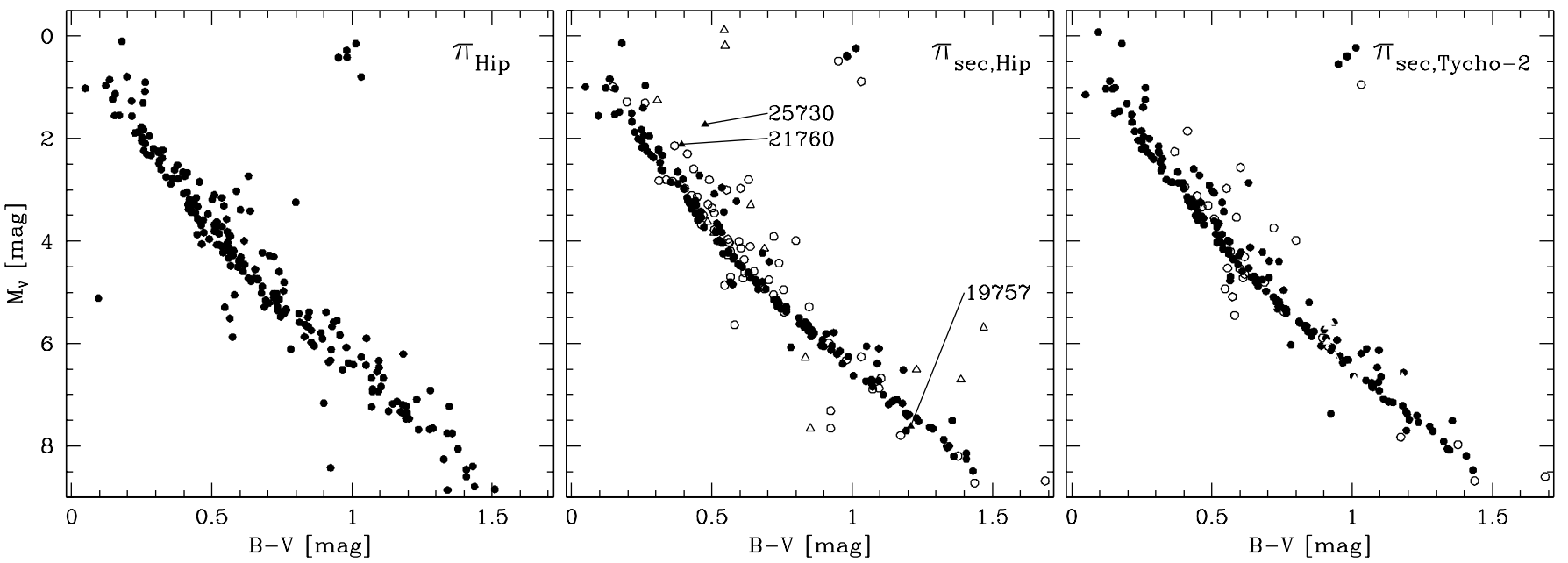

Fig. 10. Colour-absolute magnitude diagrams of the Hyades based on Hipparcos trigonometric parallaxes (left; 218 P98 members) and Hipparcos secular (middle; 218 P98 members plus 15 new candidates) and Tycho-2 secular parallaxes (right; 208 P98 members plus 23 photometric BDA members; $V$ magnitude (field H5) and $(B-V)$ colour (field H37) from the Hipparcos Catalogue). Most P98 stars below the main sequence in the left panel are "possible members" (i.e., Col. (x) is "?" in their Table 2; cf. Fig. 21 in P98). Filled symbols in the right panels have $g_{\text {Hip/Tycho-2 }} \leq 9$ while open symbols have $g_{\text {Hip } / \text { Tycho-2 }}>9$. The 15 triangular symbols in the middle panel are the new Hipparcos candidates (Sect. 5.2); the three stars with $g_{\mathrm{Hip}} \leq 9$ are labeled with their Hipparcos number. The giant region contains three(!) filled and two open symbols. The triangular symbols in the right panel represent 23 photometric BDA members (Sect. 5.3; filled triangles for $g_{\text {Tycho-2 }} \leq 9$ and open triangles for $\left.g_{\text {Tycho-2 }}>9\right)$. The giant region contains four(!) filled and one open symbol. Some faint stars $\left(V \gtrsim 8.5 \mathrm{mag}, M_{V} \gtrsim 5.2 \mathrm{mag}\right) \mathrm{in}$ the right panel have significant $(B-V)$ errors, up to several tenths of a magnitude (Sect. 8)

The maximum expected systematic error in the secular parallaxes is $\lesssim 0.3 \mathrm{mas}$ or $\lesssim 0.03 \mathrm{mag}$ (Sect. 6.5). Secular parallaxes therefore allow the construction of a well-defined and well-calibrated Hyades colour-absolute magnitude (and HR) diagram.

Figure 10 shows colour-absolute magnitude diagrams of the Hyades based on Hipparcos trigonometric (left), Hipparcos secular (middle), and Tycho-2 secular parallaxes (right). The Hipparcos secular parallax diagram shows a narrow main sequence consisting of kinematic members $\left(g_{\text {Hip }} \leq 9\right.$; filled symbols; cf. Fig. 13). Kinematically deviant stars $\left(g_{\text {Hip }}>9\right.$; open symbols) are likely either non-members and/or close multiple stars (Sect. 4.3). Most of the 15 new Hipparcos candidates (open triangular symbols; Sect. 4.2) do not follow the main sequence. This is not surprising, as secular parallaxes for most of these stars are inconsistent with their trigonometric parallaxes, suggestive of non-membership. The three candidates with $g_{\text {Hip }} \leq 9$ (filled triangles) identified in Sect. 5.2 are labeled. Only HIP 19757 lies close to the main sequence and is a likely new member (cf. Table A.2).

The right panel of Fig. 10 shows, besides a narrow cluster main sequence consisting of kinematic members ( $g_{\text {Tycho-2 }} \leq 9$; filled circles), a well-defined binary sequence for $0.45 \gtrsim(B-V) \gtrsim 0.70 \mathrm{mag}$. Most of the photometrically deviant stars are low-probability kinematic members $\left(g_{\text {Tycho-2 }}>9\right.$; open circles $)$, most likely indicating non-membership. The 23 photometric BDA members (Sect. 5.3) are indicated by triangles (filled for $g_{\text {Tycho-2 }} \leq 9$; open for $g_{\text {Tycho-2 }}>9$ ). About half of them do not follow the main sequence. Most of these objects, nonetheless, seem secure kinematic members $\left(g_{\text {Tycho-2 }} \leq\right.$ 9 ; cf. Sect. 5.3). These stars are possibly interlopers but most likely they are members with inaccurate $(B-V)$ photometry: objects lacking accurate ground-based photometric observations, most likely as a result of being preHipparcos non-members, generally have Hipparcos $(B-V)$ values derived from Tycho photometry (Hipparcos field H39 = "T"). Corresponding $(B-V)$ errors can reach several tenths of a magnitude for stars fainter than $V \sim$ $8.5 \mathrm{mag}\left(M_{V} \sim 5.2 \mathrm{mag}\right)$. Hipparcos $(B-V)$ values for faint pre-Hipparcos members contained in the Hipparcos Catalogue, on the other hand, are often carefully selected accurate ground-based measurements (field H39 = "G"); this explains the presence of a well-defined main sequence down to faint magnitudes $(V \sim 11-12 \mathrm{mag})$.

Figure 11 compares colour-absolute magnitude diagrams for different regions within the cluster (core, corona, halo, and moving group; Sect. 4.1). Secular parallaxes clearly improve the definition of the main sequence as compared to Hipparcos trigonometric parallaxes in the central parts of the cluster. They also significantly narrow the main sequence for stars in the halo $(10 \leq r<20$ pc $)$. The relatively large spread in the Hipparcos secular parallax panel for $20 \leq r<40 \mathrm{pc}$ is probably due to uncertain membership assignment (cf. Sect. 6.4.2), combined with inaccurate photometry, stellar multiplicity, and/or suspect secular parallaxes. The latter uncertainty is possibly related to unmodelled low-amplitude velocity patterns in the very outer parts of the cluster $\left(r \gtrsim 2 r_{\mathrm{t}}\right.$; but see Sects. 6.4-6.5). 


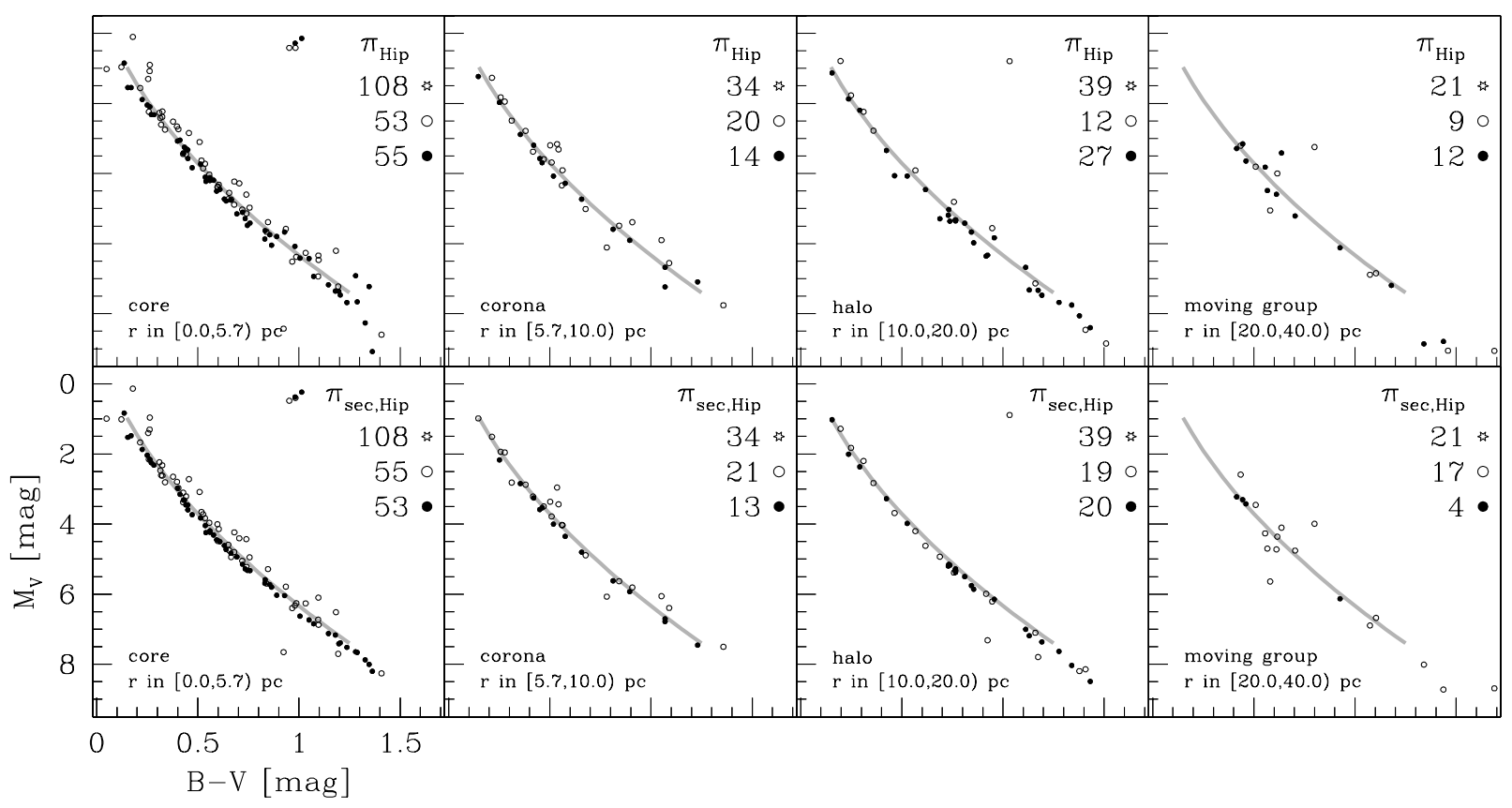

Fig. 11. Colour-absolute magnitude diagrams of the Hyades (218 P98 members) based on Hipparcos trigonometric (top row) and Hipparcos secular (bottom row) parallaxes for four different spatial regions within the cluster: the core $(r<5.7$ pc; first column), the corona $(5.7 \leq r<10 \mathrm{pc}$; second column), the halo $(10 \leq r<20 \mathrm{pc}$; third column), and the moving group population $(20 \leq r<40 \mathrm{pc}$; fourth column). In each panel, the division of stars according to their three-dimensional distance $r$ to the cluster center $\left(b_{u}, b_{v}, b_{w}\right)=(-43.37,0.40,-17.46)$ pc (in Galactic Cartesian coordinates) is based on the Hipparcos secular parallax. The gray lines outline Schwan's (1991; his Table 3) Hyades main sequence; this calibration is not necessarily the optimal one but is only shown for reference. The numbers preceding the open asterisks denote the total number of stars in each panel. Open symbols denote stars which might have "peculiar" HR diagram positions: (1) kinematically deviant stars, i.e., $g_{\text {Hip }}>9$; (2) (close) multiple stars, i.e., either Col. (s) in P98's Table 2 is "SB or RV" or Col. (u) is one of "C G OV X S"; (3) photometrically variable stars, i.e., Hipparcos field H52 is one of "D M P R U"; (4) stars with inaccurate Hipparcos $(B-V)$ photometry, i.e., $\sigma_{(B-V)}>0.05$ mag; or (5) suspect objects (HIP 20901, 21670, 20614; Sect. 9.2 in P98). Filled symbols thus denote kinematically high-fidelity photometrically non-variable single members with reliable photometry

\section{Constructing the Hertzsprung-Russell diagram}

The secular parallaxes derived in Sect. 5 constrain the locations of stars in the colour-absolute magnitude diagram with unprecedented precision (Sect. 8). An interpretation of these high-quality observations in terms of stellar evolutionary models with appropriately chosen input physics (Sect. 9.1) can provide a wealth of information on the fundamental properties of the Hyades cluster itself, such as its age and metallicity (Sect. 9.2), as well as on the characteristics of stars and stellar evolution in general (Sects. 9.3$9.4)$.

\subsection{Theoretical stellar evolutionary models}

Stellar evolutionary models have been highly successful in explaining the structure and evolution of stars (e.g., Cox \& Giuli 1968; Kippenhahn \& Weigert 1990). Numerical stellar evolutionary codes suffer from daunting practical problems, owing to, e.g., the large dynamical range for the various quantities of interest such as temperature, pressure, and density (e.g., Schwarzschild 1958; Henyey et al. 1959). Moreover, they suffer from major uncertainties in the appropriate input physics (e.g., Lebreton et al. 1995, 2000; Kurucz 2000). The most important of these uncertainties are, for Hyades main sequence stars, related to: (1) stellar atmosphere models, including issues related to atomic and molecular opacities and internal structureexternal boundary conditions (Sect. 10.1); and (2) the treatment of turbulent convection ${ }^{9}$, including issues related to convective core and envelope overshoot. Besides these two major uncertainties, numerous other physical phenomena are generally either not or only partially taken into account: (transport processes due to) stellar rotation (Sect. 10.3), variability and/or pulsational instability (Sect. 10.3), chromospheric activity (Sect. 10.1), mass loss (Sect. 10.4), binary evolution, the evolution towards the zero-age main sequence, etc. Moreover, stellar evolutionary models are generally calibrated by using the Sun as benchmark. Although the phenomenological treatment of convection in models is considered appropriate for Sunlike stars, this is not necessarily the case for stars with other mass, metallicity, and/or stellar evolutionary status. Related to the latter issue is the important open

\footnotetext{
${ }^{9}$ Corresponding physical theories do not exist; two numerical prescriptions are in wide-spread use: the Mixing-Length Theory (MLT; e.g., Böhm-Vitense 1953, 1958) and the Full Spectrum of Turbulent eddies convection model (FST; e.g., Canuto \& Mazzitelli 1991, 1992; Canuto et al. 1996).
} 
question to what extent the characteristics of convection vary with location in a convection zone. A detailed study of the secular parallax-based locations of Hyades in the colour-absolute magnitude and HR diagrams of the cluster has the potential to shed light on some of the abovementioned issues (e.g., Lebreton 2000).

\subsection{Helium content, metallicity, and age of the Hyades}

CESAM: P98 used the CESAM evolutionary code (Morel 1997) to interpret the Hipparcos HR diagram of the Hyades (their Sect. 9). In order to derive the cluster Hydrogen, Helium, and metal abundances by mass $(X \equiv$ $1-Y-Z, Y$, and $Z$, respectively), P98 fitted model zero-age main sequences to the observed trigonometric parallax-based zero-age main sequence positions of 19 single low-mass members ${ }^{10}$. By treating $Y$ and $Z$ as free parameters with the boundary condition that the inferred metal content of the Hyades be consistent ${ }^{11}$ with the mean spectroscopically determined metallicity $[\mathrm{Fe} / \mathrm{H}]=$ $+0.14 \pm 0.05$, P98 found $(X, Y, Z)=(0.716,0.260 \pm$ $0.020,0.024 \pm 0.003)$. The $\mathrm{P} 98[\mathrm{Fe} / \mathrm{H}]$ value is a wellestablished $^{12}$ quantity (cf. $[\mathrm{Fe} / \mathrm{H}] \gtrsim+0.12 \pm 0.03$ Cayrel et al. 1985; +0.13 \pm 0.02 Boesgaard 1989; Boesgaard \& Friel 1990). Unfortunately, the uncertainty on the derived Helium content of the cluster, combined with the existing uncertainty on the Helium content of the Sun (e.g., Brun et al. 1998), still prevents a definitive answer to the question whether the Helium content of the Hyades is subSolar or not (e.g., Strömgren et al. 1982; Hardorp 1982; Dobson 1990; Swenson et al. 1994; Pinsonneault et al. 1998).

After establishing the chemical composition of the Hyades, P98 derived a nuclear age $\tau=625 \pm 50-100 \mathrm{Myr}$ by fitting CESAM isochrones to the upper main sequence of the trigonometric parallax-based colour-absolute magnitude diagram (their Figs. 21-23 and Sect. 9.2; cf. $\tau=$ $600 \pm 50$ Myr from Torres et al. 1997a,c).

Padova: For an interpretation of the HR diagram of the Hyades, the latest Padova isochrones ${ }^{13}$ (Girardi et al. 2000a) offer the advantage that they include (post) red giant branch evolution.

\footnotetext{
${ }^{10}$ CESAM employs Solar-calibrated mixing-length theory $\left(\alpha_{\mathrm{MLT}}=1.64\right)$ and convective core overshoot. P98 use $(X, Y, Z)_{\odot}=(0.7143,0.2659,0.0175)$.

${ }^{11}$ For a Solar mixture of heavy elements, metallicity $Z$ and Iron-to-Hydrogen ratio $[\mathrm{Fe} / \mathrm{H}]$ (relative to Solar) are related by: $[\mathrm{Fe} / \mathrm{H}]=\log (Z / X)-\log (Z / X) \odot$. The quantity $(Z / X) \odot$ is usually taken from Grevesse \& Noels $(1993 a, b)$.

12 Some Hyades have quite deviant metallicities. E.g., the chromospherically active spectroscopic binary HIP 20577 has $[\mathrm{Fe} / \mathrm{H}]=0.00 \pm 0.03$ (Cayrel et al. 1985; Smith \& Ruck 1997). 13 The Padova code uses Solar-calibrated mixing-length theory $\left(\alpha_{\mathrm{MLT}}=1.68\right)$ and stellar-mass dependent convective core overshoot. Girardi found $(X, Y, Z)_{\odot}=(0.708,0.273,0.019)$.
}

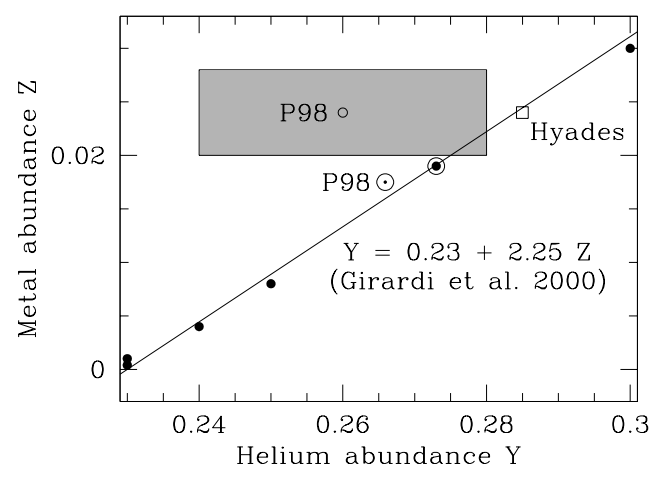

Fig. 12. The six $(Y, Z)$ values (solid dots) for which Girardi et al. (2000a) present isochrones; the solid line shows the underlying relation $Y=Y_{\mathrm{p}}+(\Delta Y / \Delta Z) \cdot Z=0.23+2.25 \cdot Z$ (Sect. 9.2). The $\odot$ symbol, which coincides with a solid dot, denotes the position of the Sun $(Y, Z)_{\odot}=(0.273,0.019)$. The open dot and corresponding gray box labeled "P98" denote the P98 Hyades value and corresponding $1 \sigma$ uncertainty; the $\odot$ symbol labeled "P98" denotes P98's Solar value $(Y, Z)_{\odot}=(0.2659,0.0175)$. The open square at $(Y, Z)=(0.285,0.024)$ labeled "Hyades" is discussed in Sect. 9.2

The six $(Y, Z)$ pairs discussed by Girardi et al. were not randomly chosen but follow a fixed $Y(Z)$ relation (Fig. 12), which is inspired by the understanding of the origin of Helium and metals in the universe: $Y \sim Y_{\mathrm{p}}+$ $(\Delta Y / \Delta Z) \cdot Z$, where $Y_{\mathrm{p}}=0.23$ is the primordial Helium abundance, and $\Delta Y / \Delta Z=2.25$ is the stellar evolution Helium-to-metal enrichment ratio (e.g., Faulkner 1967; Pagel \& Portinari 1998; Lebreton et al. 1999). The $Y(Z)$ relation implies we cannot obtain Padova isochrones which are consistent with both the Helium content and metallicity of the Hyades as derived by P98. Taking the latter fixed at $Z=0.024$, and interpolating between the sets $(Y, Z)=(0.273,0.019)$ and $(0.300,0.030)$, provides isochrones with $Y=0.285$ (open square in Fig. 12). Although this value is inconsistent at the $1.25 \sigma$ level with P98's value, it cannot be considered inappropriate for the Hyades (VandenBerg \& Bridges 1984).

As the interpolation between published isochrones is practically impossible after crossing the Hertzsprung gap, we use Girardi's Solar-metallicity $(Y, Z, \tau[\mathrm{Myr}])=$ $(0.273,0.019,631)$ isochrone for a comparison with the Hyades giants (Sect. 10.4).

\subsection{A high-fidelity stellar sample}

Following P98, we restrict attention to a high-fidelity subset of members for the study of the HR diagram. We do not consider suspect kinematic members and stars which have deviant HR diagram positions for known reasons. We exclude the 16 stars beyond 40 pc from the cluster center and all (possible) close multiple systems (98 spectroscopic binaries, Hipparcos DMSA-"G O V X S" stars, and stars with $g_{\text {Hip }}>9$ ). We furthermore reject 11 stars which are variable (Hipparcos field H52 is one of "DMPR U") or have large photometric errors $\left(\sigma_{(B-V)}>0.05 \mathrm{mag}\right)$, as 


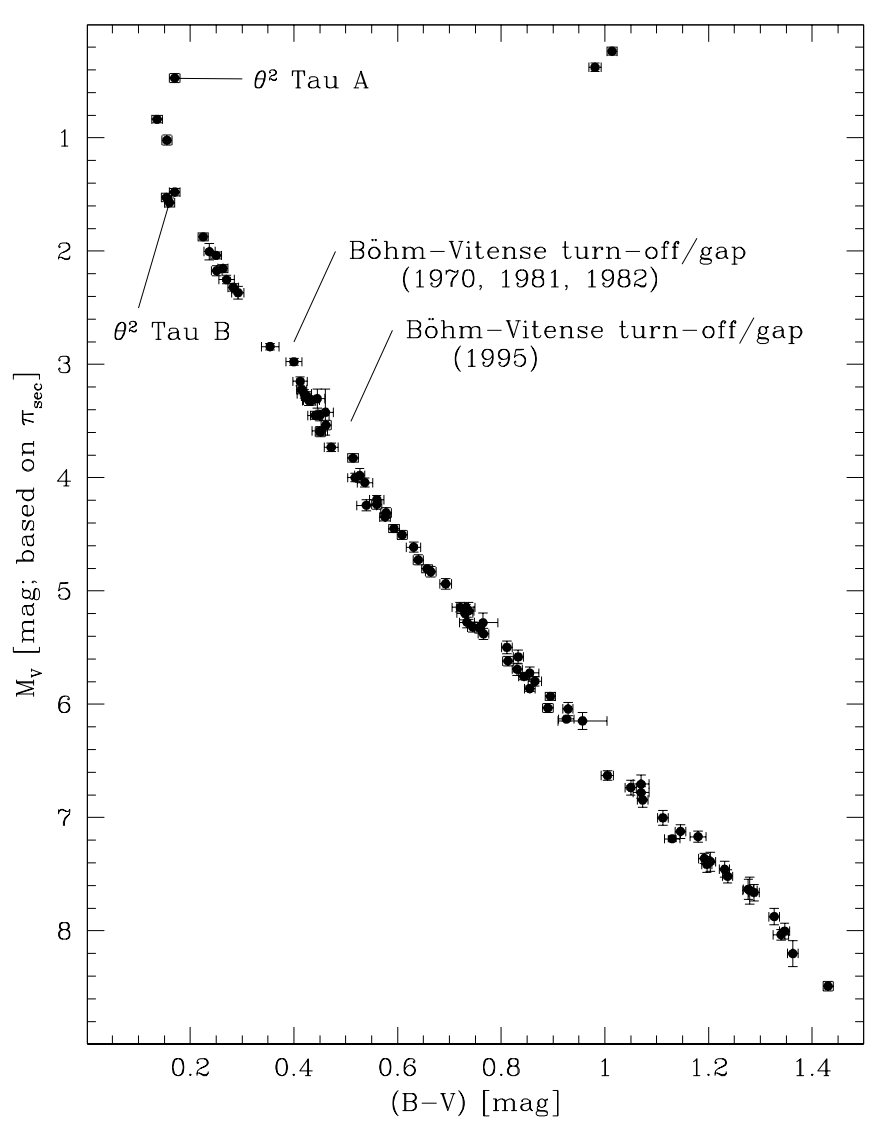

Fig. 13. Colour-absolute magnitude diagram for 92 highfidelity members (Sect. 9.3). This sample excludes all members beyond $40 \mathrm{pc}$ from the cluster center, multiple stars, and stars with suspect secular parallaxes. The absolute magnitudes have been computed using the observed $V$-band magnitudes (Hipparcos field H5) and secular parallaxes (Sect. 5; Table A.1). The $(B-V)$ colours were directly taken from the Hipparcos Catalogue (field H37). The gaps between $(B-V)=$ 0.15 and 0.20 , between $(B-V)=0.30$ and 0.35 , and the gap around $(B-V)=0.95 \mathrm{mag}$ are caused by the suppression of double, multiple, and peculiar stars from our sample (cf. Fig. 21 in P98); the region between $(B-V)=0.30$ and $(B-V)=0.35 \mathrm{mag}$, e.g., is occupied by Am-type stars, which have a high incidence of duplicity (Sect. 10.2). The lines point at two conspicuous features in the main sequence, the so-called Böhm-Vitense gaps (Sect. 10.2). The "gaps" and corresponding "turn-offs" are most likely caused by sudden changes in the properties of convective atmospheres (cf. de Bruijne et al. 2000). Figure 18a presents the corresponding theoretical HR diagram ( $\log L$ versus $\log T_{\text {eff }}$ ) for the same sample of stars

well as the suspect objects HIP 20901, 21670, and 20614 (Sect. 9.2 in P98; cf. Wielen et al. 2000).

The final sample contains 90 single members. These stars follow the main sequence (cluster isochrone) from $(B-V) \sim 1.43 \mathrm{mag}$ (late-K/early-M dwarfs; $M \gtrsim$ $\left.0.5 M_{\odot}\right)$ to $(B-V) \sim 0.10 \mathrm{mag}(\mathrm{A} 7 \mathrm{IV}$ stars; $M \sim$ $\left.2.4 M_{\odot}\right)$. Two of the stars are evolved red giants $(\epsilon$ and $\gamma$ Tau; Sect. 10.4 and B.1). The two components of the "resolved spectroscopic binary" $\theta^{2}$ Tau, located in the turnoff region of the cluster (Sects. 10.3 and B.3; cf. P98), contribute significant resolving power for distinguishing between different evolutionary models as well as between different isochrones from one evolutionary code. We therefore add them as single stars to our sample, bringing the total number of objects to 92 .

Figure 13 shows, for these 92 stars, the colour versus secular parallax-based absolute magnitude diagram (cf. Figs. 10, 11). It shows, besides a well-defined and very narrow main sequence, turn-off region, and giant clump, substructure in the form of two "gaps" / "turn-offs" in the main sequence around $(B-V) \sim 0.30$ and $\sim 0.45 \mathrm{mag}$ (cf. de Bruijne et al. 2000). These features are also present in the Tycho-2 secular parallax-based diagram (right panel of Fig. 10), but are not clearly discernible in the lower quality trigonometric parallax-based version (left panel of Fig. 10). In Sect. 10.2, we will identify these "turn-offs" with so-called Böhm-Vitense gaps, which are most likely related to convective atmospheres. Although the reality of the turn-offs in the "cleaned" secular parallax colourmagnitude diagram is hard to establish beyond all doubt, the simultaneous existence of both a turn-off and an associated gap at a location which coincides with predictions made by stellar structure models (see Sect. 10.2) strongly argues in favour of them being real (cf. Kjeldsen \& Frandsen 1991, and references therein).

\section{4. $(B-V)-M_{V} \longrightarrow \log T_{\text {eff }}-\log \left(L / L_{\odot}\right)$}

In order to compare the locations of Hyades in the HR diagram to theoretical isochrones, we need to transform the observables $(B-V)$ and $M_{V}$ to the theoretical quantities $T_{\text {eff }}$ and luminosity $L$. The usual procedure is to derive $T_{\text {eff }}$ from $(B-V)$ and then to compute the bolometric correction in the $V$-passband, $\mathrm{BC}_{V}$, from $T_{\text {eff }} ; \log \left(L / L_{\odot}\right)$ then

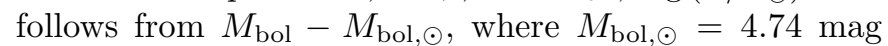
(Bessell et al. 1998; cf. footnote 16; the IAU value for the solar bolometric magnitude is $4.75 \mathrm{mag})$. Both transformations depend on metallicity $[\mathrm{Fe} / \mathrm{H}]$ and on surface gravity $\log g$, i.e., stellar evolutionary status.

\subsubsection{Previous work}

Numerous empirical and theoretical calibrations have been proposed in the past, each of which has its own validity in terms of $\log g, T_{\text {eff }},(B-V)$, and/or $[\mathrm{Fe} / \mathrm{H}]$ (e.g., Flower 1977, 1996; Buser \& Kurucz 1992; Gratton et al. 1996). There is a large uncertainty in and systematic disagreement between the different $(B-V)-T_{\text {eff }}$ relations. This is partly caused by the uncertain Solar photospheric abundances and ill-defined $(B-V)$ colour of the Sun (e.g., Appendix C in Bessell et al. 1998), but also partly by the specific choice of the $(B-V)$ index. This colour is particularly sensitive to opacity problems, mainly related to metal lines and molecular electronic transitions in the UV-blue-optical, especially for cool stars $\left(T_{\text {eff }} \lesssim 4500 \mathrm{~K}\right.$; e.g., Lejeune et al. 1998; Bessell et al. 1998). The effects of model uncertainties, related to opacities and the treatment of convection (Sect. 9.1), on theoretical calibrations 


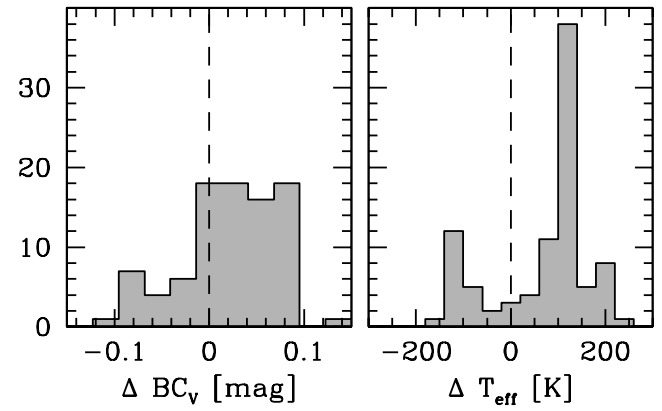

Fig. 14. The difference between bolometric corrections (left) and effective temperatures (right) derived using calibration (1) (Bessell et al. (1998) plus an Alonso et al. (1996) metallicity correction to $[\mathrm{Fe} / \mathrm{H}]=+0.14$ ) and calibration (2) (Lejeune et al. (1998) for $[\mathrm{Fe} / \mathrm{H}]=+0.14)$ for 92 high-fidelity members (Sect. 9.3), excluding the giants $\epsilon$ and $\gamma$ Tau; $\Delta$ is defined as calibration (1) minus (2). Most stars with $\Delta T_{\text {eff }}<0 \mathrm{~K}$ have $(B-V) \geq 1.0 \mathrm{mag}$, indicating that calibrations (1) and (2) differ systematically with effective temperature itself

are often non-negligible, especially for $\mathrm{K} / \mathrm{M}$ giants and low-mass dwarfs (e.g., Blackwell et al. 1991; cf. Table 4 in Houdashelt et al. 2000). The main reason for our ignorance is the lack of a representative set of stars with (model-)independently determined effective temperatures (e.g., Code et al. 1976; Ridgway et al. 1980; Blackwell \& Lynas-Gray 1994). Differences between effective temperature scales, established by empirical or theoretical calibrations, are generally smaller than $\sim 200-400 \mathrm{~K}$ (Castelli 1999; Gardiner et al. 1999).

We consider two calibrations: (1) Bessell et al. (1998) in combination with Alonso et al. (1996; Sect. 9.4.2), roughly following P98; and (2) Lejeune et al. (1998; Sect. 9.4.3).

\subsubsection{Calibration (1)}

Bessell et al. (1998) present broad-band colour, bolometric correction, and effective temperature calibrations for $\mathrm{O}$ to M stars, based on Kurucz's (1995) ATLAS9 model atmospheres. Their Tables ${ }^{14} 1$ and 3 relate $T_{\text {eff }}, \log g,(B-V)$, and $\mathrm{BC}_{V}$, based on Solar-metallicity models with convective core overshoot ${ }^{15}\left(\alpha_{\mathrm{MLT}}=1.25\right)$.

\footnotetext{
14 Tables 4 and 5 are valid for giants, which are treated separately in Sect. 10.4. Table 6 is based on NMARCS Mdwarf models. Although this calibration is preferred over the ATLAS9-based relation for stars with $T_{\text {eff }} \lesssim 4000 \mathrm{~K}$, the modeled range in $(B-V)$ is incompatible with the colours for dwarfs in our sample. Moreover, the NMARCS relations do not link smoothly to the ATLAS9 results. We therefore decided not to use them.

15 This choice is consistent with the results derived by P98 (their Sect. 9.2), although we note that Castelli et al. (1997) found that effective temperatures measured by means of the infra-red flux method (e.g., Blackwell et al. 1990) for stars with $T_{\text {eff }} \gtrsim T_{\text {eff }, \odot} \sim 5765 \mathrm{~K}$ are in general better reproduced by theoretical ATLAS9 atmosphere models (Kurucz 1995) with the envelope overshoot option switched off than with the option switched on (Table 2 in Bessell et al. 1998).
}

We use an iterative scheme to determine $\log T_{\text {eff }}$, $\log \left(L / L_{\odot}\right)$, and $\log g$ from the measured $(B-V)$ and $M_{V}$ values, in which we correct for the non-Solar metallicity of the Hyades according to Alonso et al. (1996; their Eq. (1)). Step 1 involves the determination of $\log T_{\text {eff }}$ for $[\mathrm{Fe} / \mathrm{H}]=$ 0 , from a given $\log g$ and measured $(B-V)$. After differentially applying Alonso's metallicity correction for $[\mathrm{Fe} / \mathrm{H}]=+0.14$ in step 2 , the corrected $\log T_{\text {eff }}$ provides $\mathrm{BC}_{V}$, and thus $\log \left(L / L_{\odot}\right)$. Step 3 involves the determination of $\log g=\log \left(4 \pi G M \sigma_{\text {Boltzmann }} T_{\text {eff }}^{4} L^{-1}\right)$ using stellar masses $M$ from P98 (Sect. 9.4.5). This recipe is repeated (typically 3 times) until convergence is achieved in the sense that $\log g$ remains constant; the final results do not depend on the initial estimate $\log \left(g\left[\mathrm{~cm} \mathrm{~s}^{-2}\right]\right)=4.5$.

First-order error analysis allows an estimation of the uncertainties on the derived quantities. We assume that $\sigma_{\log \left(L / L_{\odot}\right)}$ is influenced by both $\sigma_{M_{V}}$ and $\sigma_{\mathrm{BC}_{V}}$, which in their turn are influenced by $\sigma_{V}$ plus $\sigma_{\pi}$ and $\sigma_{\log T_{\text {eff }}}$, respectively. We assume that $\sigma_{\log T_{\text {eff }}}$ is influenced by both $\sigma_{(B-V)}=\min \left(\sigma_{(B-V) \text {,observed }}, 0.010 \mathrm{mag}\right)$ and $\sigma_{M}=$ $0.10 M_{\odot}$ (P98); we neglect the contribution of $\sigma_{\log g}$ to $\sigma_{\log T_{\text {eff }}}$ as it is typically an order of magnitude smaller than the other contributors (cf. Castelli et al. 1997).

\subsubsection{Calibration (2)}

Lejeune et al. (1998) present (semi-)empirical calibrations linking $T_{\text {eff }},(B-V), \log g$, and $\mathrm{BC}_{V}$ for $[\mathrm{Fe} / \mathrm{H}]$ between -3.5 and +1.0 based on BaSeL spectral energy distributions $^{16}$ (Basel Stellar Library version 2.0; their Tables 110). In the range of stellar parameters considered here, these calibrations use Kurucz (1995) ATLAS9 model atmospheres. The presentation of the data, which is relevant for dwarfs ( $\log g \gtrsim 3.75$ ), allows a direct determination of $\log T_{\text {eff }}, \log \left(L / L_{\odot}\right)$, and $\log g$ from the measured $(B-V)$ and $M_{V}$ for $[\mathrm{Fe} / \mathrm{H}]=+0.14$ by means of interpolation between Table $1([\mathrm{Fe} / \mathrm{H}]=0)$ and Table $9([\mathrm{Fe} / \mathrm{H}]=+0.50)$, without relying on stellar mass information. We derive $\sigma_{\log \left(L / L_{\odot}\right)}$ and $\sigma_{\log T_{\text {eff }}}$ as in Sect. 9.4.2.

\subsubsection{Results for dwarfs}

We applied calibrations (1) and (2) to the set of 92 members described in Sect. 9.3, excluding the giants $\epsilon$ and $\gamma$ Tau (Appendix B.1), using absolute magnitudes $M_{V}$ based on Hipparcos secular parallaxes (Sect. 5). Details for the spectroscopic binary $\theta^{2}$ Tau are given in Appendix B.3.

For a given calibration, the bolometric corrections are relatively well defined, except at lower masses (i.e., redder $(B-V)$, lower $T_{\text {eff }}$; Figs. 14, 15). Effective temperatures, on the other hand, are quite uncertain, especially at higher masses. Moreover, a comparison of the

\footnotetext{
16 Lejeune uses $M_{V, \odot}=4.854 \mathrm{mag}$ and $\mathrm{BC}_{V, \odot}=-0.108 \mathrm{mag}$; we have transformed Lejeune's data to conform with Bessell's zero point (cf. Appendices C-D in Bessell et al. 1998).
} 

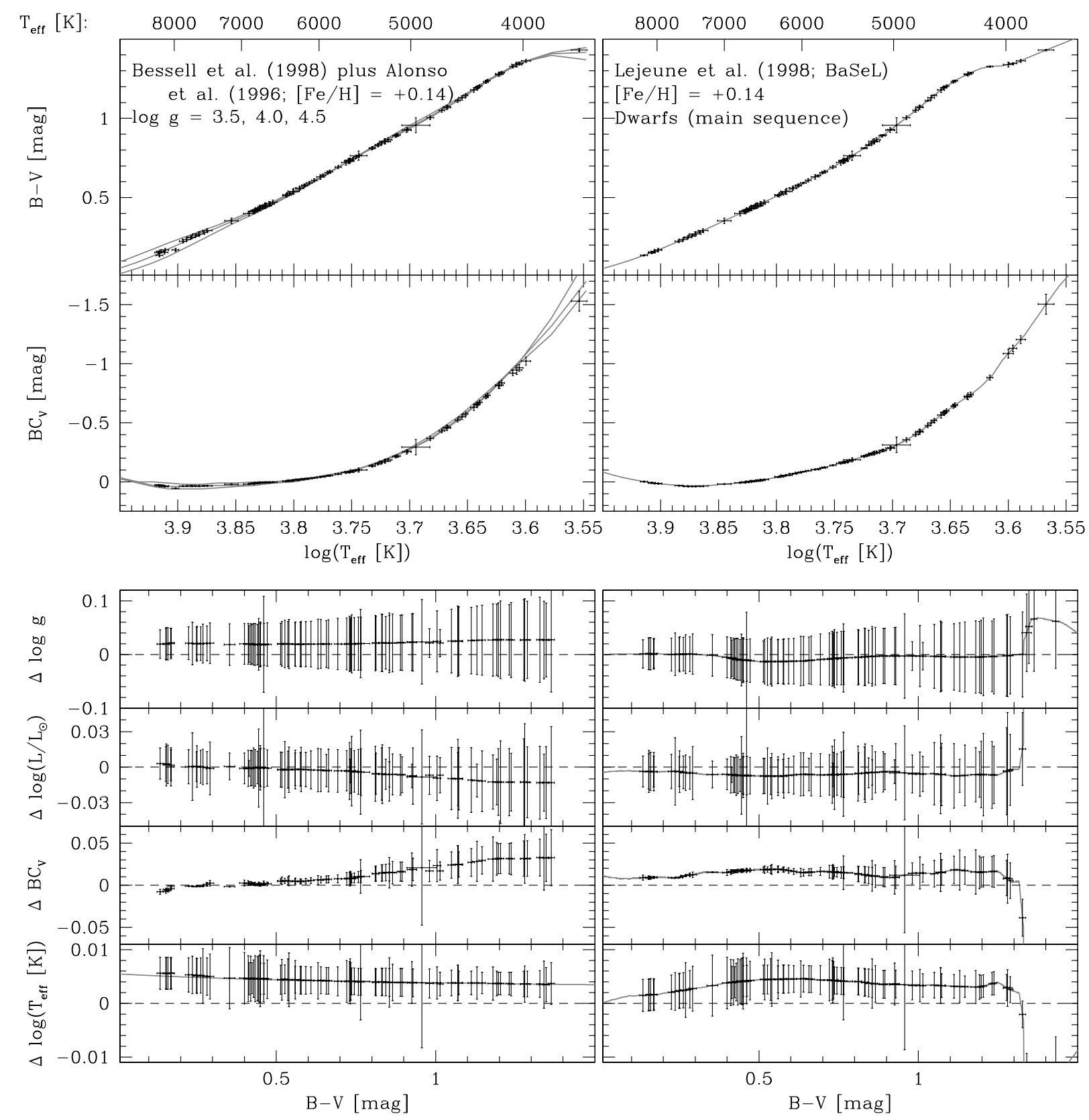

Fig. 15. Theoretical quantities $\log T_{\text {eff }}, \mathrm{BC}_{V}, \log \left(L / L_{\odot}\right)$, and $\log g$ derived from $(B-V)$ and $M_{V}$ (the latter based on Hipparcos secular parallaxes) using Bessell et al. (1998) plus Alonso et al. (1996; left column) and Lejeune et al. (1998; right column; Sects. 9.4.2-9.4.3). Left column: the gray curves in the top two panels show the Bessell et al. relation for $[\mathrm{Fe} / \mathrm{H}]=0$ and $\log g=3.5,4.0$, and 4.5 (from bottom to top along the vertical line $\log \left(T_{\text {eff }}[\mathrm{K}]\right)=3.9$, respectively); the (tiny) dots and crosses show the (metallicity corrected) values and uncertainties for the 92 high-fidelity Hyades (Sect. 9.3), excluding the giants $\epsilon$ and $\gamma$ Tau. The bottom four panels show the effect of correcting the Bessell et al. calibration for the non-Solar Hyades metallicity $[\mathrm{Fe} / \mathrm{H}]=+0.14$ on (from the top panel down) $\log g, \log \left(L / L_{\odot}\right), \mathrm{BC}_{V}$, and $\log T_{\text {eff }}$. The difference $\Delta x$ is defined as $\Delta x \equiv x_{[\mathrm{Fe} / \mathrm{H}]=+0.14}-x_{[\mathrm{Fe} / \mathrm{H}]=0}$ for $x=\log g$, etc. The faint M dwarf HIP $15720((B-V)=1.431 \pm 0.004$ mag $)$ has no Bessell et al. solution for $[\mathrm{Fe} / \mathrm{H}]=0$. The gray line in the bottom panel follows Alonso's Eq. (1). Right column: as left column, but using the Lejeune et al. relation for dwarfs with $[\mathrm{Fe} / \mathrm{H}]=+0.14$. The bottom four panels show the effect of going from $[\mathrm{Fe} / \mathrm{H}]=0$ to $[\mathrm{Fe} / \mathrm{H}]=+0.14$ by means of interpolation. The apparent discontinuities of the gray lines in the lower panels are caused by the peculiar behaviour of the Lejeune et al. $[\mathrm{Fe} / \mathrm{H}]=+0.50$ relation (their Table 9$)$ around $(B-V)=1.30$ mag. Several stars with $(B-V)>1.30 \mathrm{mag}$ fall outside the plotted ranges in the bottom panels

effective temperatures derived from calibrations (1) and (2) reveals significant systematic differences (at the level of $\sim 100 \mathrm{~K}$; Fig. 14) as a function of $T_{\text {eff }}$ itself. Taking the
non-Solar metallicity of the Hyades into account significantly changes the derived parameters, and notably the effective temperatures (Fig. 15). 


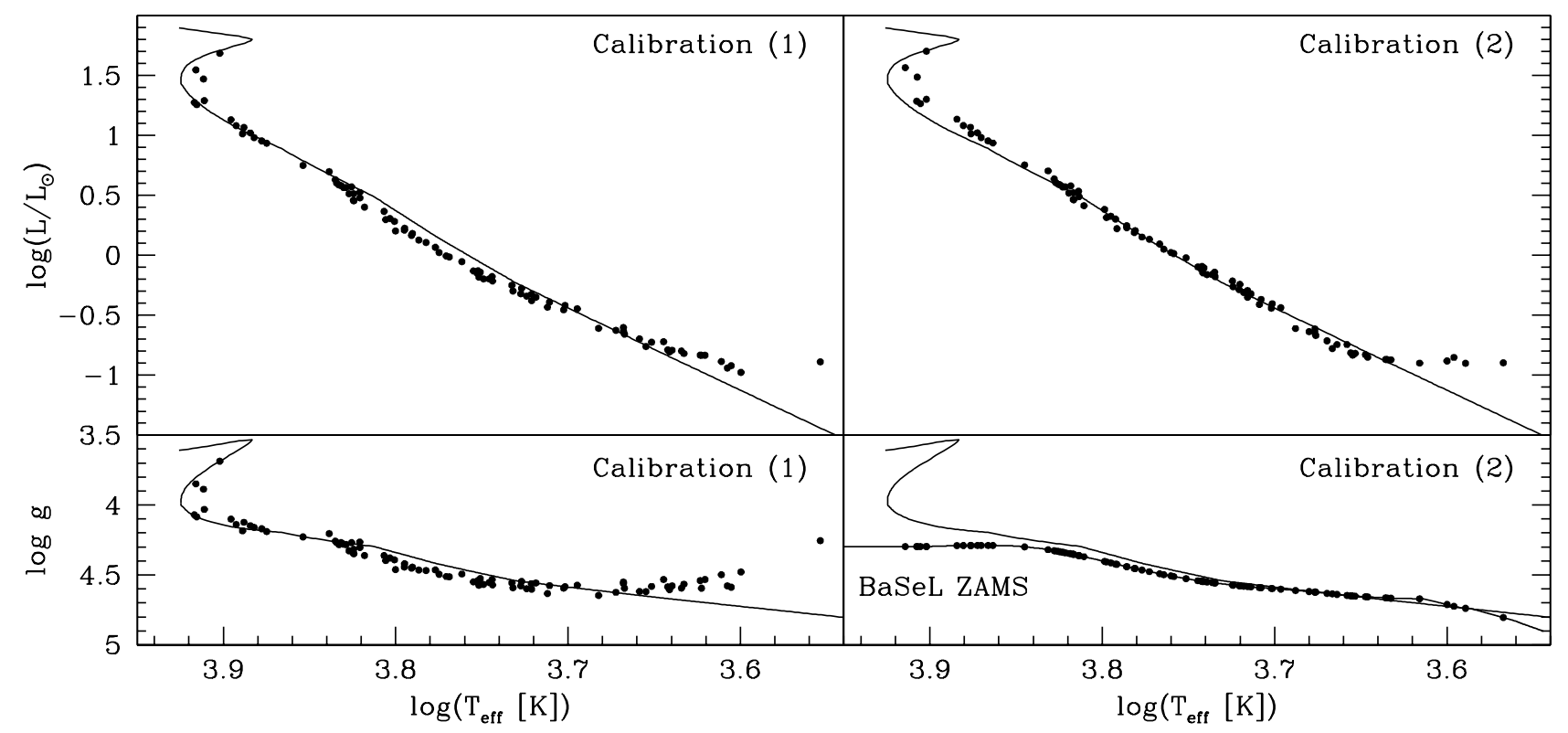

Fig. 16. The $\log T_{\text {eff }}-\log \left(L / L_{\odot}\right)($ top $)$ and $\log T_{\text {eff }}-\log g$ (bottom) diagrams for the same 92 stars as in Figs. 18a and 18b, excluding the giants $\gamma$ and $\epsilon$ Tau, but using calibration (1) (Sect. 9.4.2; left) and (2) (Sect. 9.4.3; right) for all stars. The curves denote the 625 Myr CESAM isochrones

In order to establish which calibration is to be preferred, we compared the effective temperatures following from Bessell's and Lejeune's relations (for $[\mathrm{Fe} / \mathrm{H}]=0$ as well as $[\mathrm{Fe} / \mathrm{H}]=+0.14)$ with a number of previously established effective temperature scales for the Hyades (Bessell et al. [1998; with and without overshoot; see footnote 15] plus Alonso et al. [1996; $[\mathrm{Fe} / \mathrm{H}]=0$ and +0.14$]$; Lejeune et al. [1998; $[\mathrm{Fe} / \mathrm{H}]=0$ and +0.14$]$; Allende Prieto \& Lambert [1999; Table 1]; Varenne \& Monier [1999; Table 2]; P98 [Table 8]; and Balachandran [1995; Table 4]). This analysis reveals effective temperature differences, which sometimes vary systematically with effective temperature itself (cf. Fig. 14), up to $\sim 300 \mathrm{~K}$. Unfortunately, none of these scales is truly fundamental in the sense of having been established completely modelindependently. In fact, agreement between different calibrations might even be artificial to some degree, as several of them have ultimately been calibrated using Kurucz atmosphere models (e.g., Gardiner et al. 1999). We therefore decided to enforce consistency between the calibration used here and the spectroscopic effective temperatures and CESAM isochrones provided by $\mathrm{P} 98$.

Figure 16 compares the $\log T_{\text {eff }}-\log \left(L / L_{\odot}\right)$ and $\log$ $T_{\text {eff }}-\log g$ diagrams after applying calibration (1) and calibration (2) to all objects in the sample. Calibration (2) has the problem that evolved stars in the turn-off region (i.e., luminosity classes $\mathrm{IV}-\mathrm{V}$ ) are given too large surface gravities because the procedure assumes that all stars are dwarfs (i.e., luminosity class V). Calibration (1) has the "problem" that stars on the main sequence fall significantly below the $625 \mathrm{Myr}$ CESAM isochrone, whereas stars in the turn-off region of the cluster follow this curve acceptably well. These facts suggest the use of calibration (2) for dwarfs ${ }^{17}$ and calibration (1) for the 14 stars with $(B-V) \leq 0.300 \mathrm{mag}\left(T_{\text {eff }} \geq 7250 \mathrm{~K}\right.$; $\left.\log \left(T_{\text {eff }}[\mathrm{K}]\right) \geq 3.8603\right)$. We acknowledge that this approach is "ad hoc", as it naively assumes the CESAM isochrones are correct. A full understanding of the discrepancies shown in Fig. 16 requires a set of new isochrones and calibrations which are tailored to the Hyades in terms of metallicity and Helium content. The construction of such isochrones and calibrations, using the secular parallax-based $(B-V)-M_{V}$ diagram presented in Fig. 13 as boundary condition, is beyond the scope of this paper (cf. Sect. 11).

\subsubsection{Stellar masses}

Figure 17 compares the masses (Sect. 5.3 in P98) of the 92 high-fidelity members and the Hipparcos secular parallaxbased absolute magnitudes with an empirical mass-absolute magnitude relation derived for the Hyades (Eq. (A3) in Patience et al. 1998). The empirical relation deviates significantly from the P98 masses for low-mass dwarfs $\left(M \lesssim 1 M_{\odot} ;\right.$ cf. Sect. 10.1). The CESAM and Padova isochrone results (Sect. 9.2), on the other hand, give an acceptable "fit". This confirms that the P98 masses, which are used in calibration (1) to derive $T_{\text {eff }}$ and $\log \left(L / L_{\odot}\right)$ from $(B-V)$ and $M_{V}$ (Sect. 9.4.2), are well-defined.

\section{Hertzsprung-Russell diagram}

Figure 18 shows 92 high-fidelity Hyades (Sect. 9.3) with Padova and CESAM isochrones (Sect. 9.2) in the

$\overline{17}$ We find $T_{\text {eff }, \odot}=5793 \mathrm{~K}, \log \left(L / L_{\odot}\right)=0.004$, and $\log g=$ 4.501 for $(B-V) \odot=0.628 \mathrm{mag}$ (Taylor 1998). 


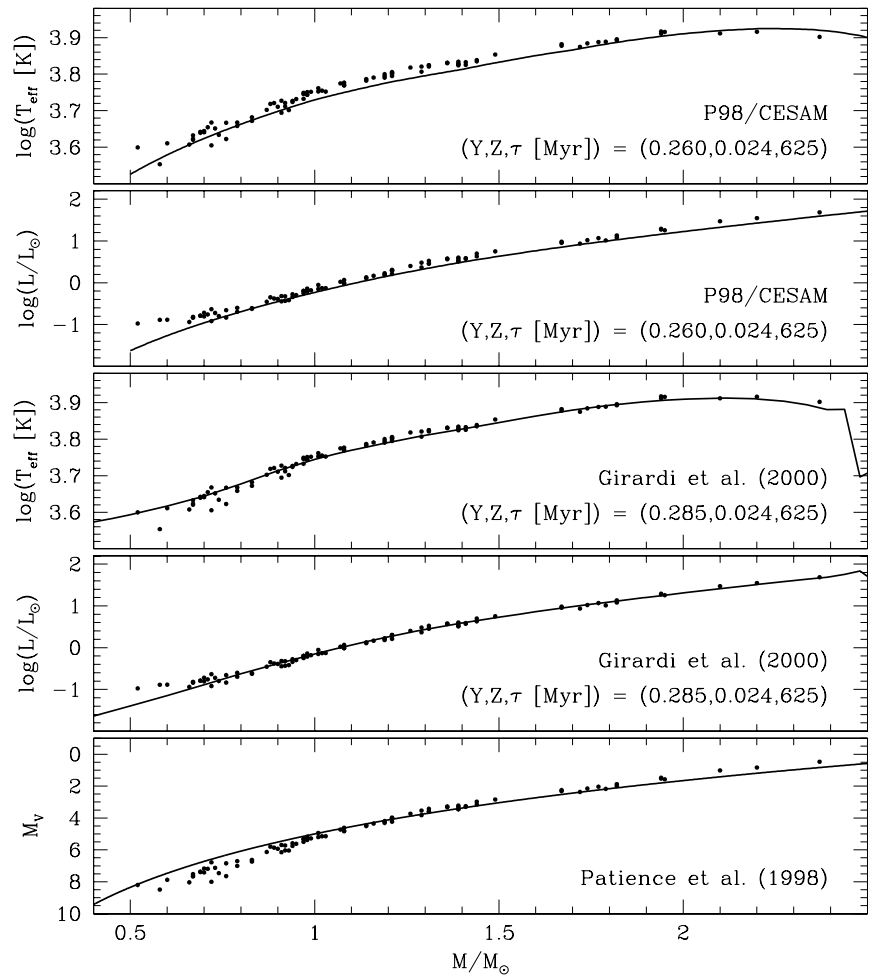

Fig. 17. Masses of 92 high-fidelity members (Sect. 9.4.5), excluding the giants $\epsilon$ and $\gamma$ Tau. The most massive stars in this figure are (in order of decreasing mass): HIP $20894 \mathrm{~A}\left(\theta^{2}\right.$ Tau A; Appendix B.3), 20635, and 23497. Top two panels: the solid lines denote the effective temperature- and luminosity-stellar mass relations of the $(Y, Z)=(0.260,0.024) 625$ Myr CESAM isochrone (P98; Sect. 9.2). Third and fourth panels: as the top two panels, but for the $(Y, Z)=(0.285,0.024) 625$ Myr Padova isochrone (Girardi et al. 2000a). Bottom panel: the empirical absolute magnitude-mass relation of Patience et al. (1998) compared with the P98 stellar masses. The absolute magnitudes are based on Hipparcos secular parallaxes (Sect. 5)

theoretical HR diagram (panel a) and the $\log T_{\text {eff }}-\log g$ diagram (panel b). We used calibration (1) for the 14 evolved stars in the cluster turn-off region $((B-V) \leq 0.30$ mag; $T_{\text {eff }} \geq 7250 \mathrm{~K}$; Sect. 9.4.2) and calibration (2) for the remaining 76 dwarfs $\left((B-V)>0.30 \mathrm{mag} ; T_{\text {eff }}<7250 \mathrm{~K}\right.$; Sects. 9.4.3-9.4.4).

\subsection{Low-mass main sequence $\left(M \lesssim 0.9 M_{\odot}\right)$}

Figure 18a shows, besides an inconsistency between the Padova and CESAM isochrones themselves, a significant discrepancy between the isochrones on the one hand and the inferred effective temperatures and luminosities for stars with $T_{\text {eff }} \lesssim 5000 \mathrm{~K}$ (K/M dwarfs) on the other hand. As the "turn-off" of the lower masses is absent in the colour-absolute magnitude diagram (Fig. 13), we conclude that the model-observation discrepancies for these cool stars are caused by inappropriate $(B-V)-T_{\text {eff }}$ calibrations (e.g., Gratton et al. 1996; cf. Sect. 9.4.1 and 9.4.4,
Figs. 17-16, and footnote 15). Reliable transformations for low-mass dwarfs with cool envelopes (or completely convective interiors) require non-gray non-LTE line-blanketed model atmospheres with appropriate molecular opacities (e.g., TiO, $\mathrm{H}_{2} \mathrm{O}$, and VO; e.g., Hauschildt et al. 1999). Theoretical models in this mass range similarly require adequate boundary conditions (Chabrier \& Baraffe 1997). These models, for $M \lesssim 0.6 M_{\odot}$, also suffer from uncertainties related to the equation of state, and, for $4000 \lesssim$ $T_{\text {eff }} \lesssim 5000 \mathrm{~K}$, from uncertainties related to the treatment of convection (e.g., Ludwig et al. 1999).

The low-mass stars as a set show an enhanced spread about the main sequence compared to the higher mass stars. This effect is partly related to the degradation of secular parallax (i.e., Hipparcos proper motion) accuracies with $V$ magnitude. Part of the scatter might also be due to spectral peculiarities (e.g., emission lines as in HIP 20527 [K5.5Ve], 20605 [M0.5Ve], and 21138 [K5Ve]) or colour anomalies related to chromospheric activity (e.g., Campbell 1984; Stauffer et al. 1991, 1997); a 5 per cent starspot coverage yields $\sim 0.015 \mathrm{mag}$ shifts in $(B-V)$, either towards the blue or towards the red, as well as lowlevel photometric variations (on the order of a few hundredths of a magnitude).

\subsection{Intermediate-mass main sequence $\left(0.9 \lesssim M \lesssim 1.6 M_{\odot}\right)$}

This mass range corresponds to $\mathrm{F}$ and $\mathrm{G}$ stars $(7500 \gtrsim$ $\left.T_{\text {eff }} \gtrsim 5000 \mathrm{~K}\right)$. We divide it into three regimes corresponding to different interiors: (1) Stars with $M \gtrsim 1.5 M_{\odot}$ $\left(B-V \lesssim 0.30 \mathrm{mag} ; T_{\text {eff }} \gtrsim 7000 \mathrm{~K}\right)$ have a convective core and radiative envelope. (2) With decreasing mass in the range $1.5 \gtrsim M \gtrsim 1.3-1.1 M_{\odot}(0.30 \lesssim B-V \lesssim 0.40 \mathrm{mag}$; $7000 \gtrsim T_{\text {eff }} \gtrsim 6500-6000 \mathrm{~K}$ ), the convective core shrinks to become radiative, while a convective envelope develops at the same time. This envelope gives rise to the formation of a chromosphere and corona. (3) Stars with $M \lesssim 1.3-1.1 M_{\odot}\left(B-V \gtrsim 0.40 \mathrm{mag} ; T_{\text {eff }} \lesssim 6500-6000 \mathrm{~K}\right)$ have a radiative core and convective envelope. Theoretical modelling for these regimes suffers mainly from uncertainties related to convection, notably overshoot of the core and envelope, and the issue of the universality of the mixing-length parameter $\alpha_{\mathrm{MLT}}$ (e.g., Ludwig et al. 1999; Sect. 9.1).

Figure 18a shows that the effective temperatures and luminosities closely follow the CESAM isochrone, except in the range $7000 \gtrsim T_{\text {eff }} \gtrsim 6500 \mathrm{~K}(0.30 \lesssim(B-V) \lesssim$ $0.40 \mathrm{mag})$ where the observations are suggestive of a "turn-off" of the main sequence around spectral type $\sim$ F5V. Figure 19 shows an expanded view of this region, including the locations of all non-high-fidelity members. The "turn-off" is also clearly visible in the secular parallax-based colour-absolute magnitude diagrams displayed in Figs. 11 and 13. We therefore conclude that it is not caused by the $(B-V)-T_{\text {eff }}$ relation adopted 


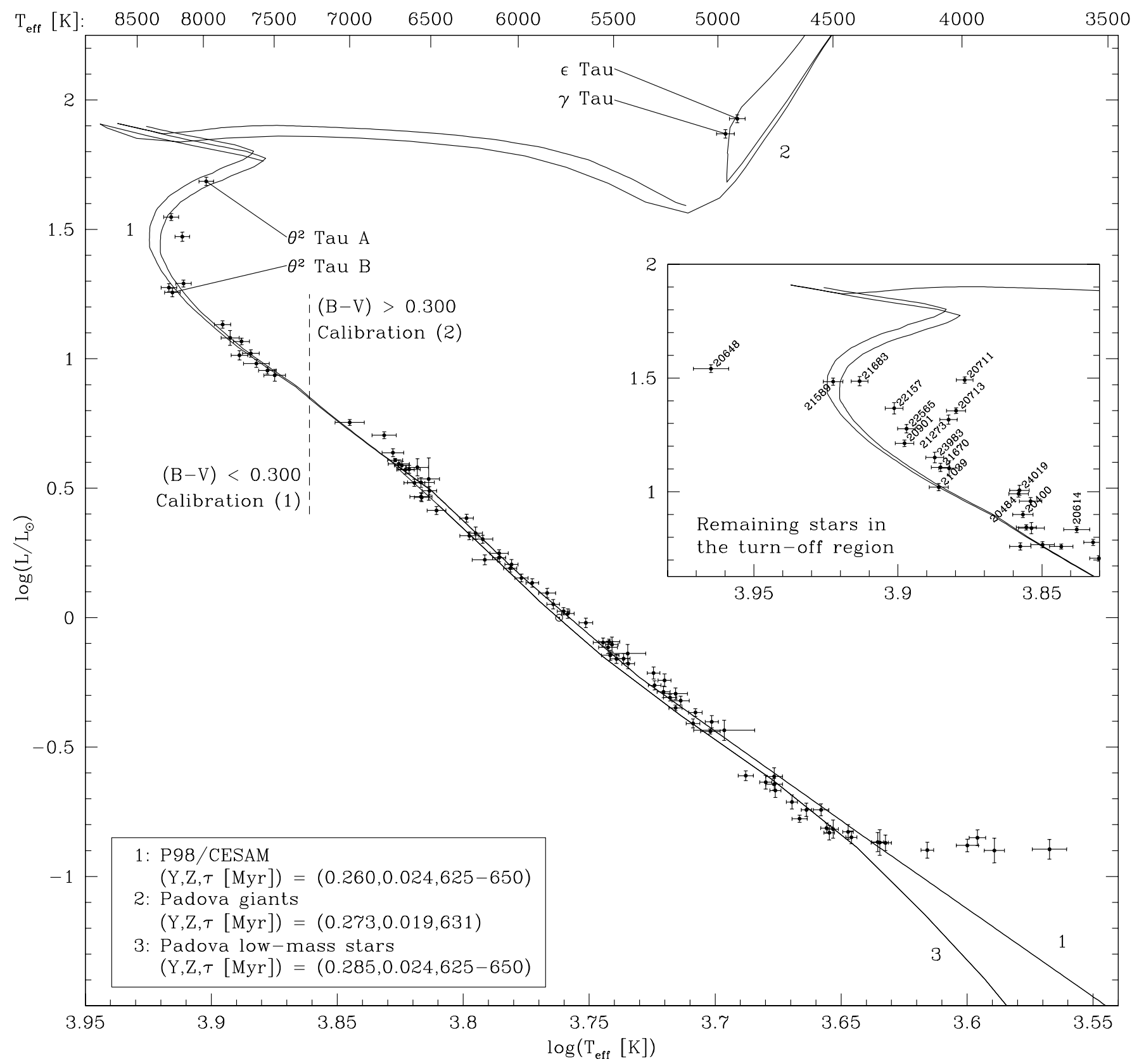

Fig. 18. Panel a) HR diagram for 92 high-fidelity Hyades (Sect. 9.3). The stellar luminosities and effective temperatures were derived from secular parallax-based absolute magnitudes $M_{V}$ and $(B-V)$ colours using calibration $(1)$ for $(B-V) \leq 0.300$ mag $\left(T_{\text {eff }} \geq 7250 \mathrm{~K} ; 14\right.$ stars; Sect. 9.4.2) and calibration $(2)$ for $(B-V)>0.300 \mathrm{mag}\left(T_{\text {eff }}<7250 \mathrm{~K} ; 76\right.$ dwarfs; Sect. 9.4.3). The giants $\gamma$ and $\epsilon$ Tau (HIP 20205 and 20889) are discussed in Appendix B.1; the components of the "resolved spectroscopic binary" $\theta^{2}$ Tau (HIP 20894) are discussed in Appendix B.3. The lines labeled "1", "2", and " 3 " are CESAM and Padova isochrones for the Hyades with Helium content $Y$, metal content $Z$, and age $\tau$ as indicated in the box (Sect. 9.2). The position of the Sun is indicated by $\odot$. The deviant locations of cool stars $\left(T_{\text {eff }} \lesssim 5000 \mathrm{~K}\right)$ are a result of systematic errors in the $(B-V)-T_{\text {eff }}$ calibrations (Sect. 10.1; cf. Fig. 15). The inset shows the remaining members in the upper main sequence and turn-off region of the cluster (excluding HIP 21459, which has large photometric errors). HIP 20648 is discussed in Sect. 10.3. Deviant locations can be attributed to multiplicity, peculiar spectra, rotation, and/or suspect secular parallaxes $\left(g_{\mathrm{Hip}}>9\right.$; Table 4$)$

in this study (Sect. 9.4.4; cf. Fig. 16; we do note, however, that the "turn-off" roughly coincides with the transition at $(B-V)=0.30 \mathrm{mag}\left(T_{\text {eff }} \sim 7250 \mathrm{~K}\right)$ between calibration (1) (Sect. 9.4.2) and calibration (2) (Sect. 9.4.3)).
We suspect, as argued below, that the "turn-off" is related to the onset of surface convection around $(B-V)=0.30$ 0.40 mag (cf. de Bruijne et al. 2000). This is consistent with the work of Rachford (1997, 1998), who detected 


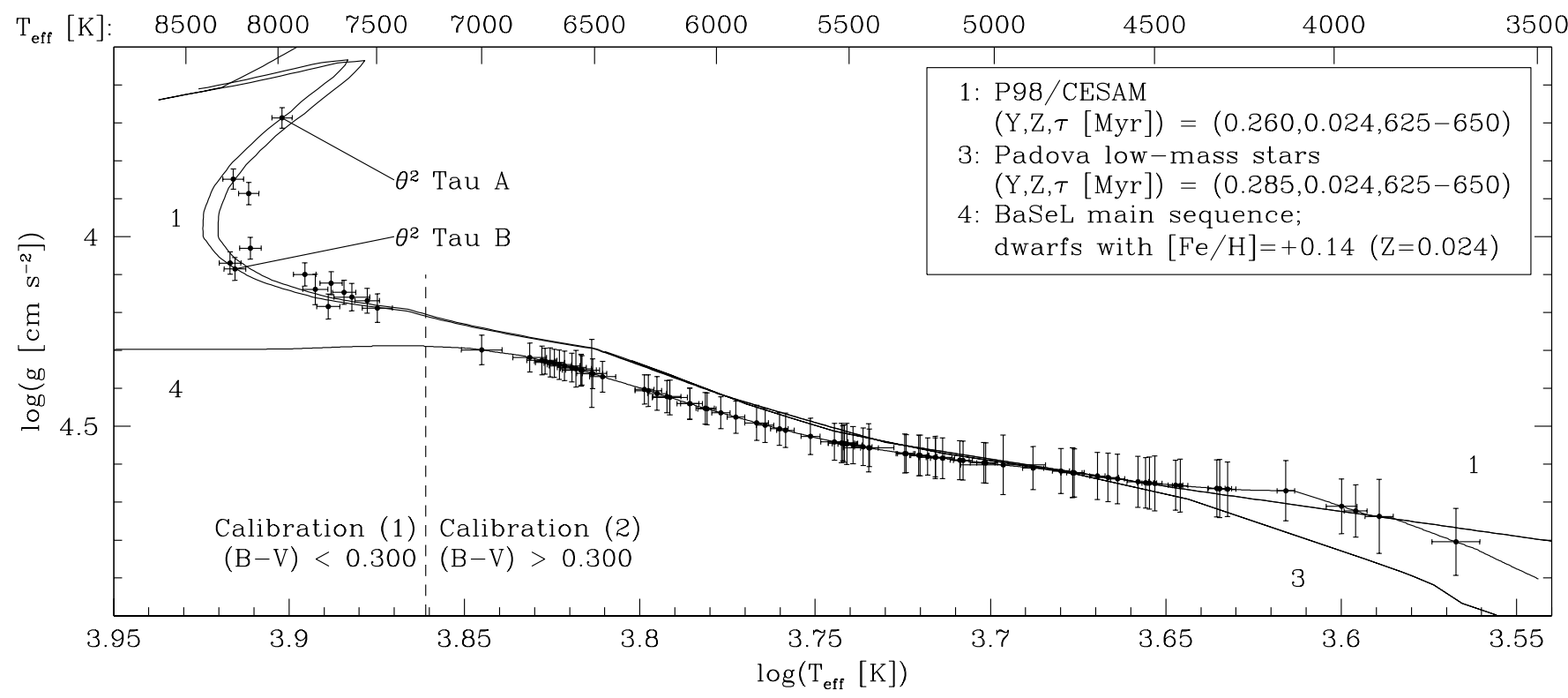

Fig. 18. Panel b) The $\log T_{\text {eff }}-\log g$ diagram for the same 92 stars as in panel a), but excluding the giants $\gamma$ and $\epsilon$ Tau. The line labeled "4" denotes the BaSeL calibration main sequence for $[\mathrm{Fe} / \mathrm{H}]=+0.14(Z=0.024$; Sect. 9.4.3). Bessell et al.'s (1998) calibration (Sect. 9.4.2) is differently organized and cannot be plotted in this diagram

a transition in stellar activity ${ }^{18}$ parameters at $(B-V) \sim$ $0.29 \mathrm{mag}$ as well as a chromospherically active Hyad at $(B-V)=0.26$ mag (HIP 21036). These findings indicate that the onset of convection (in the Hyades) occurs at $(B-V) \gtrsim 0.25-0.30 \mathrm{mag}$ (cf. Wolff et al. 1986; Rachford $\&$ Canterna 2000).

Böhm-Vitense $(1970,1981,1982)$ first realized that convective atmospheres have relatively low temperatures in the deeper layers which contribute to the surface flux in the spectral regions of the $U$ and $B$ filters (cf. Nelson 1980). As a result, convective atmospheres have reddened $(B-V)$ colours (as compared to radiative atmospheres of the same $T_{\text {eff }}$ ) by amounts of $\sim 0.07-0.12$ mag. As the reddening of the atmosphere is not accompanied by a significant change in luminosity, the onset of surface convection can cause a $\sim 0.10 \mathrm{mag}$ "gap" and/or "turn-off" in the $(B-V)$ colour-absolute magnitude diagram, starting around $(B-V)=0.25-0.35 \mathrm{mag}$, the so-called Böhm-Vitense gap ${ }^{19}$ (cf. Fig. 2 in de Bruijne et al. 2000). Observational evidence for the existence of the Böhm-Vitense gap is sparse (e.g., Böhm-Vitense \& Canterna 1974; Jasniewicz 1984; Rachford \& Canterna 2000), and its reality has been disputed (e.g., Mazzei \& Pigatto 1988; Simon \& Landsman 1997; Newberg \& Yanny 1998). Previous claims for its existence were based on the

18 The onset of surface convection is accompanied by X-ray and near-UV emission from coronal and chromospheric gas, which in its turn is due to magnetic fields produced by a stellar rotation-induced dynamo (e.g., Pallavicini et al. 2000).

19 The location of this gap roughly coincides with the Amtype stars. As these objects are often close multiples (e.g., Jaschek \& Jaschek 1987; Debernardi et al. 2000), most of them are not contained in the high-fidelity sample (Sect. 9.3), causing a reduced sampling of the main sequence (Figs. 18a and 19). presence of gaps in either colour-colour diagrams or in the cumulative distribution of cluster members in some photometric index (e.g., Aizenman et al. 1969), instead of on the presence of "gaps" or "turn-offs" in colour-absolute magnitude diagrams. The secular parallax-based colourabsolute magnitude diagrams of the Hyades presented in Figs. 11 and 13 provide, in fact, the first direct evidence in favor of the existence of the Böhm-Vitense gap.

A careful inspection of Figs. 11, 13, and 18a reveals that the region around $(B-V) \sim 0.45 \mathrm{mag}\left(T_{\text {eff }} \sim 6400 \mathrm{~K}\right)$ also shows an abrupt increase in the $(B-V)$ colours of stars by an amount of $\sim 0.05$ mag. This feature was already commented on by Böhm-Vitense (1995a,b), who attributed it to " a sudden increase in convection zone depths". The position of the second Böhm-Vitense gap coincides with both the so-called Lithium gap, which is generally thought to be related to the rapid growth of the depth of the surface convection zone with effective temperature decreasing from $\sim 7000 \mathrm{~K}$ to $\sim 6400 \mathrm{~K}$ (e.g., Boesgaard \& Tripicco 1986; Michaud 1986; Swenson et al. 1994; Balachandran 1995), and the onset of dynamoinduced magnetic chromospheric activity (cf. Wolff et al. 1986; García Lopéz et al. 1993).

As stellar rotation is known to influence significantly the precise conditions for the onset of surface convection (e.g., Chandrasekhar 1961), Böhm-Vitense suggested that a range of $v \sin i$ values within a stellar cluster can lead to a bifurcation of the $(B-V)-T_{\text {eff }}$ relation in the range $6500 \lesssim T_{\text {eff }} \lesssim 7500 \mathrm{~K}$ (cf. Simon \& Landsman 1997). This means that a star with a given $(B-V)$ colour can be either a slowly rotating weakly convective star with a "high" effective temperature or a rapidly rotating radiative star with a "lower" effective temperature, where the 


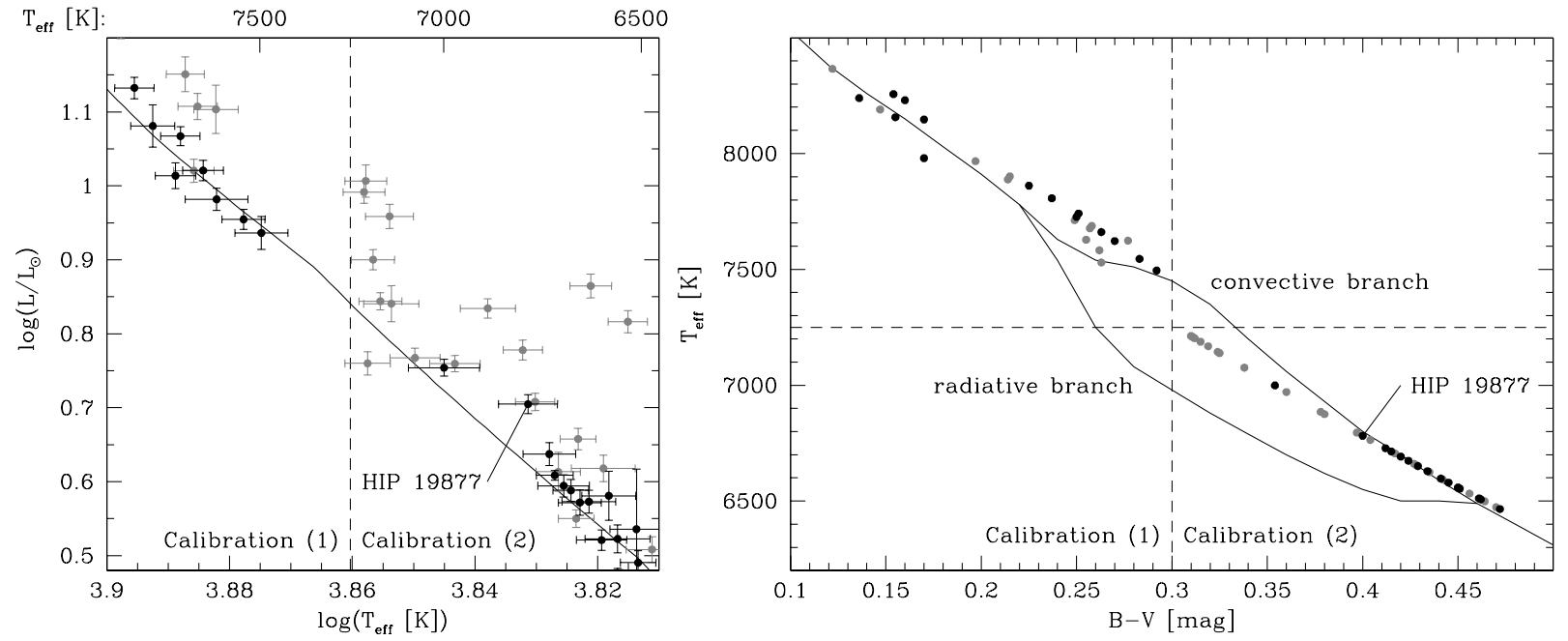

Fig. 19. Left: HR diagram of the region of the onset of surface convection (the Böhm-Vitense gap; Sect. 10.2). The solid line denotes the 625 Myr CESAM isochrone. Black symbols show high-fidelity members; gray symbols denote remaining members (excluding HIP 21459, which has large photometric errors). Although the rotational velocity of the deviant object HIP 19877 (F5var) is unknown, its detection in X-rays (Hünsch et al. 1998) suggests the star has a convective envelope. Patience et al. (1998) did not detect a speckle companion. Its location above the main sequence is hence possibly related to stellar variability $(\Delta V \sim 0.07 \mathrm{mag})$ or activity. The dashed line denotes $(B-V)=0.30 \mathrm{mag}\left(T_{\text {eff }} \sim 7250 \mathrm{~K}\right)$, which marks the boundary between effective temperatures derived using calibrations (1) and (2) (Sects. 9.4.2-9.4.3). Right: effective temperatures versus $(B-V$ ) colours for the same set of stars as shown in the left panel. The dashed horizontal line denotes $T_{\text {eff }}=7250 \mathrm{~K}$, and is shown for reference. The solid line shows the Böhm-Vitense (1981) Solar-metallicity $(B-V)-T_{\text {eff }}$ relation; the bifurcation into radiative and convective branches is discussed in Sect. 10.2

Table 4. Some remaining stars in the upper main sequence and turn-off region of the Hyades. Spectroscopic binarity ("SB"; Table 2 in P98), high rotational velocities ( $v \sin i$ from Abt \& Morrell 1995; $\left.\mathrm{km} \mathrm{s}^{-1}\right)$, suspect secular parallaxes $\left(g_{\mathrm{Hip}}>9\right)$, and spectral peculiarities can give rise to deviant HR diagram positions. Hipparcos field H52 is a photometric variability flag ("M" for micro-variable). The symbol $\delta$ Sct stands for $\delta$ Scuti pulsator

\begin{tabular}{|c|c|c|c|c|c|c|c|c|c|c|c|}
\hline HIP & SB & $v \sin i$ & Notes & HIP & $\mathrm{SB}$ & $v \sin i$ & Notes & HIP & $\mathrm{SB}$ & $v \sin i$ & Notes \\
\hline 20400 & SB & 25 & Am; $\delta$ Sct & 20901 & & 93 & $\mathrm{Am}$ & $21683^{\mathrm{c}}$ & & 115 & $g_{\mathrm{Hip}}=11.92 ; \mathrm{A} 5 \mathrm{Vn}$ \\
\hline 20484 & SB & 15 & $\mathrm{Am}$ & 21039 & SB & 31 & Am & 22157 & SB & 63 & $\mathrm{Am} ; g_{\mathrm{Hip}}=23.34$ \\
\hline $20614^{\mathrm{a}}$ & & 145 & & 21273 & SB & 130 & & 22565 & & 165 & $\mathrm{H} 52=$ "M"; $\delta$ Sct \\
\hline 20711 & & 225 & $\mathrm{H} 52=$ "M" $; \delta$ Sct $; \mathrm{A} 8 \mathrm{Vn}$ & 21589 & SB & 78 & & 23983 & SB & 13 & $\mathrm{Am}$ \\
\hline $20713^{\mathrm{b}}$ & SB & 205 & $g_{\text {Hip }}=30.30 ; \delta$ Sct & 21670 & & 75 & $\mathrm{Am}$ & $24019^{\mathrm{d}}$ & & 45 & $\mathrm{Am}$ \\
\hline
\end{tabular}

a: Eggen's (1992) "photometry indicates a possible binary, which is not resolved by speckle observations". Patience et al. (1998) did not detect a speckle companion. Wielen et al. (2000) list the star as " $\Delta \mu$ binary".

${ }^{\mathrm{b}}$ : A chromospherically active long-period F0V+G4V binary (Peterson et al. 1981); the object is the second-brightest X-ray source in the Hyades (e.g., Stern et al. 1992, 1994).

${ }^{\mathrm{c}}$ : Parallaxes differ significantly $\left(\pi_{\mathrm{Hip}}=20.51 \pm 0.82, \pi_{\mathrm{sec}, \mathrm{Hip}}=18.33 \pm 0.42, \pi_{\mathrm{sec}, \text { Tycho }-2}=18.70 \pm 0.38 \mathrm{mas} ; g_{\mathrm{Hip} / \text { Tycho }-2}=\right.$ 11.92/8.74), possibly as a result of multiplicity. Patience et al. (1998) did not detect a speckle companion.

d: Primary target of a two-pointing system (field H60 = "P"); the A component is a periodic variable. The secondary target is HIP 24020; the double star processing used a linear system $(\mathrm{H} 60=$ "L"): the parallax of the B component is constrained to be identical to the A-component parallax, whereas the proper motions were independently determined. The solution is uncertain $(\mathrm{H} 61=$ "D"). HIP 24020 is a duplicity-induced variable $(\mathrm{H} 52=$ "D"); the B component itself is a resolved Hipparcos binary.

"high" and "low" temperatures can differ by as much as 500 K. The righthand panel of Fig. 19 compares BöhmVitense's (1981; her Table 3 and Fig. 4) predictions of the two branches to the effective temperatures derived using calibrations (1) and (2) (Sects. 9.4.2-9.4.3). The calibration (1) and (2) effective temperatures do not show a bifurcation, simply because they do not take stellar rotation into account. Systematic differences outside the bifurcation region can be due to the presently outdated Solarmetallicity atmosphere models used by Böhm-Vitense in 1981. A natural next step would be to determine, for each Hyades member in the region of the (first) Böhm-Vitense gap, its rotational velocity, to determine its effective temperature based on adequate stellar modelling, i.e., taking 
stellar rotation into account, and to compare the resulting location in the $T_{\text {eff }}-(B-V)$ diagram (Fig. 19) with the predictions of Böhm-Vitense (1981).

Naively, one would expect that the peculiar $(B-V)$ behaviour resulting from (the onset of) surface convection is incorporated in the (synthetic) $(B-V)-T_{\text {eff }}$ relations adopted in this paper. However, as the onset of surface convection is a subtle effect when translated into the mass range at stake, its visibility in the theoretical HR diagram (Fig. 18a) is probably the result of an inappropriate mass sampling in the construction of the synthetic $(B-V)-T_{\text {eff }}$ relations (Sects. 9.4.2-9.4.3). Only a future investigation of the interplay between stellar rotation and (the onset of) surface convection, and the corresponding effects on the atmospheric parameters of mid-F stars (e.g., Hartmann \& Noyes 1987; Chaboyer et al. 1995), can shed more light on the issue of the "turn-off(s)" of the Hyades main sequence and the bifurcation of the $(B-V)-T_{\text {eff }}$ relation.

\subsection{The upper main sequence and turn-off region $\left(M \gtrsim 1.6 M_{\odot}\right)$}

Beyond $T_{\text {eff }} \gtrsim 7500 \mathrm{~K}$, we find the early-F and late-A stars which are evolving towards the end of the core Hydrogen burning phase. These objects are powered by the CNOcycle and have convective cores and radiative envelopes. As a result, the precise locations of the isochrones depend strongly on the treatment of rotationally-induced mixing (Maeder \& Meynet 2000) and the amount of convective core overshoot included in the models (e.g., Sect. 9.2 in P98). Given these uncertainties, Fig. 18 shows that the stars on the upper main sequence of the Hyades follow the 625-650 Myr CESAM isochrones (including convective core overshoot) remarkably well, both in luminosity (panel a) and in surface gravity (panel b).

Many stars in the turn-off region of the Hyades rotate rapidly (e.g., Table 11 in P98). Stellar rotation influences the observed colours and magnitudes (and thus the inferred temperatures and luminosities) of stars, the amounts depending on the rotation characteristics (solidbody or differential rotation), the rate of rotation, the orientation of the rotation axis with respect to the observer, and the spectral type of the star (e.g., Kraft \& Wrubel 1965; Maeder \& Peytremann 1970, 1972; Collins \& Smith 1985; Pérez Hernández et al. 1999; Maeder \& Meynet 2000). Although the photometric effect of rotation also depends on the specific filter system in use, rotating stars generally become redder (typically a few hundredths of a magnitude in $(B-V) ; \sim 200-250 \mathrm{~K}$ in $\left.T_{\text {eff }}\right)$ as well as brighter or fainter (typically a few tenths of a magnitude in $V$ ). An example of a rapidly rotating star in the Hyades turn-off region is HIP $23497\left(v \sin i \sim 126 \mathrm{~km} \mathrm{~s}^{-1}\right.$; $\left.\log \left(T_{\text {eff }}[\mathrm{K}]\right)=3.9115 ; \log \left(L / L_{\odot}\right)=1.472\right)$; its deviant location in the HR diagram is most likely due to rotation: although the object is listed as equal-magnitude occultation double by Hoffleit \& Jaschek (1991), Patience et al. (1998) did not detect a secondary.
The turn-off region of the Hyades coincides with the lower part of the instability strip (e.g., Liu et al. 1997). Some of the stars accordingly pulsate (e.g., Antonello \& Pasinetti Fracassini 1998). An example is the primary component of the "resolved spectroscopic binary" $\theta^{2}$ Tau (HIP 20894), which is a $\delta$ Scuti pulsator (e.g., Breger et al. 1987, 1989; Kennelly et al. 1996; cf. Appendix B.3 and Table 3). The A7III-IV primary has an A5V secondary. The exact location of both stars in the turn-off region of the HR diagram therefore puts a severe constraint on stellar evolutionary models of the system as well as of the Hyades cluster (e.g., Lastennet et al. 1999). Unfortunately, the precise evolutionary status of the evolved component is unknown. Moreover, observations as well as theoretical modelling are complicated by the fact that both stars, which are roughly equally bright, are rapid rotators $\left([v \sin i]_{\text {primary }} \sim 80 \mathrm{~km}^{-1} ;[v \sin i]_{\text {secondary }} \gtrsim 90 \mathrm{~km}^{-1}\right)$, resulting in severely blended spectra.

The secular parallax of the binary allows to position both components in the HR diagram with unprecedented precision. Figure 18a suggests that $\theta^{2}$ Tau A is close to the end of the core Hydrogen burning phase, and is about to undergo an overall gravitational contraction which will ignite thick Hydrogen shell burning. This is consistent with the low amplitude of the pulsations, indicative of main sequence evolution (e.g., Li \& Michel 1999; large-amplitude pulsators (0.10 mag or higher) are thought to be evolved stars in the Hydrogen shell burning phase). These results confirm the conclusion of Torres et al. (1997c), which was based on the orbital parallax of the binary (Table 3), but are in conflict with Królikowska's (1992) finding that $\theta^{2}$ Tau A currently burns Hydrogen in a thick shell.

The bluest Hyad is HIP 20648 (A2IVm; $(B-V)=$ $0.049 \pm 0.007 \mathrm{mag})$. Being a Hipparcos duplicity-induced variable (field H52 is " $\mathrm{D}$ ") and component binary (H59 is "C"; $\Delta H p=4.02 \pm 0.09 \mathrm{mag})$, the object is not included in our high-fidelity sample. Nonetheless, the object is a secure member of the Hyades: its secular parallax solution (based on the Hipparcos A-component proper motion; H10 is " $\mathrm{A}$ ") is of high quality $\left(g_{\mathrm{Hip}}=0.46\right)$, and the observed radial velocity and parallax (secular as well as trigonometric) are consistent with membership (e.g., Table 2 in P98). Applying calibration (1) to the observed colour and secular parallax returns $\log \left(T_{\text {eff }}[\mathrm{K}]\right)=3.9649 \pm 0.0061$ and $\log \left(L / L_{\odot}\right)=1.541 \pm 0.017$. These parameters put the star on the Hyades main sequence, but far beyond the turn-off (inset of Fig. 18a). This "blue straggler" nature is not readily explained by rapid rotation (since $v \sin i=15 \mathrm{~km} \mathrm{~s}^{-1}$ and a pole-on orientation is unlikely) or an inappropriate calibration (e.g., Burkhart \& Coupry (1989) found $\left.T_{\text {eff }}=9050 \pm 100 \mathrm{~K}\right)$. The observation of a large photospheric (and thus presumably also large interior) magnetic field (Babcock 1958) suggests that the apparently prolonged hydrogen core burning phase of this star, as compared to other members of similar mass which have already evolved off the main sequence, can be explained if the Helium produced in the core is continuously 


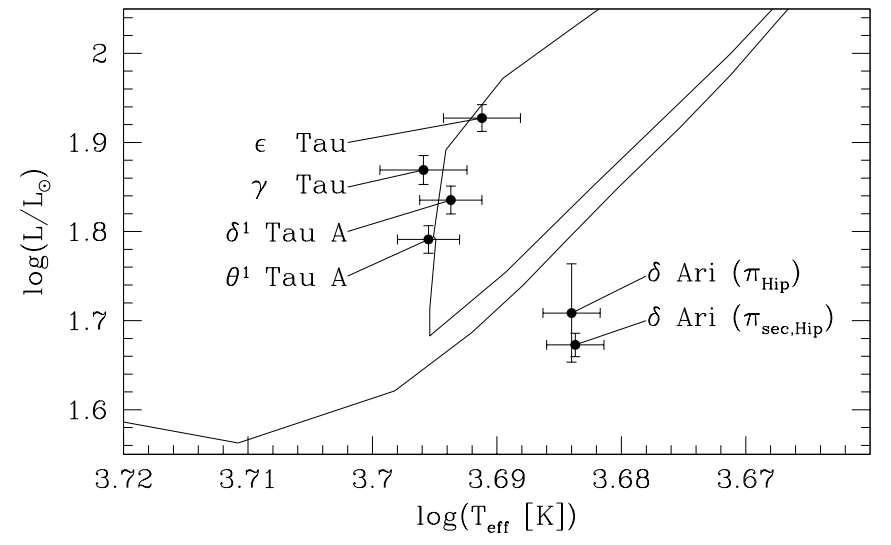

Fig. 20. The Hyades red giant clump based on Hipparcos secular parallaxes (Appendices B.1-B.2). The solid line denotes Girardi et al.'s (2000a) $(Y, Z)=(0.273,0.019) 631 \mathrm{Myr}$ isochrone. The objects $\delta^{1}$ and $\theta^{1}$ Tau are spectroscopic binaries; their secular parallaxes and luminosities should be treated with care (Sect. 10.4). The "suspected non-single" K2III giant $\delta$ Ari is not coeval with the Hyades; this conclusion does not depend on the suspect secular parallax of the star (Sect. 10.4)

replaced with fresh Hydrogen through large-scale magnetic mixing (e.g., Hubbard \& Dearborn 1980; Abt 1985).

The inset of Fig. 18a shows all objects in the turnoff region of the Hyades which are not contained in the objectively defined sample of high-fidelity members (cf. Table 4). The deviant HR diagram locations of some of these members are most likely caused by: (1) suspect secular parallaxes (i.e., $g_{\text {Hip }}>9$ ); (2) stellar rotation; (3) (spectroscopic) binarity; or (4) an inappropriate calibration (Sect. 9.4). The latter option might be especially relevant for the slowly rotating metallic-line A stars. Uncertainties in (metal-)line-blanketing, line lists, and opacities in atmosphere models, the treatment of the diffusion of chemical elements, and the treatment of largescale motions in the envelopes of these stars give rise to

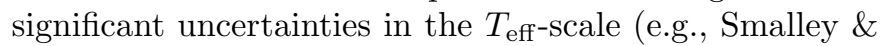
Dworetsky 1993; Richer et al. 2000).

\subsection{The giant region}

The red clump of the Hyades contains four giants: $\theta^{1}, \delta^{1}$, $\epsilon$, and $\gamma \mathrm{Tau}$; the determination of their effective temperatures and secular parallax-based luminosities is discussed in Appendices B.1-B.2 Whereas $\epsilon$ and $\gamma$ Tau are single stars, $\theta^{1}$ and $\delta^{1}$ Tau are spectroscopic binaries. The secular parallaxes of these double stars should be treated with care as they could be based on astrometric data which do not properly reflect their true space motions (Sect. 4.3).

The location of isochrones in the giant region not only depends quite sensitively on metallicity but also on the mass loss history on the red giant branch and the adopted value of the mixing-length parameter $\alpha_{\mathrm{MLT}}$. Nonetheless, all Hyades giants precisely follow Girardi et al.'s (2000a) Solar-metallicity $631 \mathrm{Myr}$ isochrone (Sect. 9.2; Fig. 20), despite the fact that Girardi simply accounted for mass loss by means of Reimers' (1975) empirical formula (using a mass-loss efficiency parameter of 0.4; Renzini \& Fusi Pecci 1988). A natural next step would be to investigate the variation of the location of the red giant clump with metallicity, mass loss, and mixing-length parameter (cf. Girardi et al. 2000b). Such an analysis would give, e.g., more insight into the reliability of red clump giants as distance calibrators (e.g., Alves 2000; Udalski 2000).

The P98 member list contains one additional evolved star of spectral type K2III. This object ( $\delta$ Ari; HIP 14838; Appendix B.2) has several characteristics of a nonmember: it is located $15.34 \mathrm{pc}$ from the cluster center, it has a near-Solar metallicity as opposed to the mean Hyades value $[\mathrm{Fe} / \mathrm{H}]=+0.14 \pm 0.05$, it has an unreliable secular parallax solution, and it was rejected by de Bruijne (1999a) and Hoogerwerf \& Aguilar (1999) as proper motion member of the Hyades (Table A.1). Although the latter two facts could be spurious due to possible duplicity (Appendix B.2; Wielen et al. 2000), Fig. 20 shows that $\delta$ Ari is not coeval with the classical giants in the cluster. We conclude that the star is most likely a non-member.

\subsection{White dwarfs}

The Hyades contains a dozen ${ }^{20}$ known white dwarfs (e.g., Humason \& Zwicky 1947; Luyten 1954, 1956; BöhmVitense 1995). They typically have $V \gtrsim 14 \mathrm{mag}$, which means they are too faint to be observed directly by Hipparcos. However, several white dwarfs are contained in multiple systems (e.g., Lanning \& Pesch 1981), the primary components of which were observed by Hipparcos. We discuss two of such systems.

V471 Tau: HIP 17962 is a post-common-envelope detached eclipsing binary. It is composed of a DA white dwarf and a coronally active K2V star. Hosting the hottest and youngest Hyades white dwarf, V471 Tau has been studied extensively (e.g., Nelson \& Young 1970, 1972; Guinan \& Sion 1984; Clemens et al. 1992; Shipman et al. 1995; Marsh et al. 1997). The system is a pre-cataclysmic variable: the $\mathrm{K}$ star does not fill its Roche lobe yet. The observed periodic optical and X-ray variations are related to material from the $\mathrm{K}$-star wind being accreted onto the magnetic poles of the rotating white dwarf (e.g., Jensen et al. 1986; Barstow et al. 1992).

The effective temperature and surface gravity of the white dwarf in V471 Tau were recently determined by fitting synthetic spectra to observed spectra of the Hydrogen Lyman lines (Barstow et al. 1997; Werner \& Rauch 1997;

\footnotetext{
${ }^{20}$ The present-day luminosity function of the Hyades predicts the cluster contains $\sim 25-30$ white dwarfs (e.g., Chin \& Stothers 1971). The discrepancy between the observed and predicted number of these objects is possibly explained by evaporation from the cluster (e.g., Weidemann et al. 1992; Eggen 1993; Sect. 4.1). One possible example of an escaped white dwarf is the P98 candidate HIP 12031 (DAwe...). It is located beyond $40 \mathrm{pc}$ from the cluster center, and is possibly a kinematic member $\left(g_{\text {Hip }}=0.80 ; g_{\text {Tycho }-2}=12.61\right)$.
} 
Table 5. Fundamental parameters of two Hyades white dwarfs. V471 Tau: effective temperatures and surface gravities determined from ORFEUS/IUE spectra (Barstow et al. 1997; Werner \& Rauch 1997). The orbital elements of the eclipsing binary imply a mass function of $0.174 \pm 0.002 M_{\odot}$; using $80^{\circ} \leq i \leq 90^{\circ}$ returns an astrometric white dwarf mass $M=0.759 \pm 0.020 M_{\odot}$ for an assumed K-dwarf mass $M=0.800 M_{\odot}$ (Young 1976; Bois et al. 1988). The white dwarf radius $R$ is independently determined from model fluxes and the observed parallax and flux. The inferred surface gravity then follows from combining this radius with the astrometric mass. Barstow et al. used Wood's (1995) evolutionary models to derive a spectroscopic mass $M / M_{\odot}=0.61_{-0.10}^{+0.14}$ and radius $R / R_{\odot}=0.014 \pm 0.003$. "This study" combines the secular parallax of V471 Tau with Werner \& Rauch's model. HD 27483: results from Böhm-Vitense (1993) and Burleigh et al. (1998). The Burleigh et al. and "This study" results were derived from an interpolation in Burleigh's Table 5 using the Hipparcos trigonometric and secular parallax, respectively. Burleigh et al. themselves quote $\log g=8.5, T_{\text {eff }}=22000 \mathrm{~K}$, and $M / M_{\odot}=0.94$

\begin{tabular}{lrrr}
\hline \hline V471 Tau & Barstow et al. \\
HIP 17962 & $\left(1997 ; \pi_{\text {Hip }}\right)$ & $\begin{array}{r}\text { Werner \& Rauch } \\
\left(1997 ; \pi_{\text {Hip }}\right)\end{array}$ & $\begin{array}{r}\text { This study } \\
\left(\pi_{\text {sec,Hip }}\right)\end{array}$ \\
\hline$T_{\text {eff }}[\mathrm{K}]($ spectroscopic $)$ & $32400_{-800}^{+270}$ & $35125 \pm 1275$ & \\
$\log \left(g\left[\mathrm{~cm} \mathrm{~s}^{-2}\right]\right)$ (spectroscopic) & $8.16_{-0.24}^{+0.18}$ & $8.21 \pm 0.23$ & $0.759 \pm 0.020$ \\
$M / M_{\odot}($ from orbit) & $0.76 \pm 0.02$ & $0.759 \pm 0.020$ & $0.0098 \pm 0.0011$ \\
$R / R_{\odot}($ from parallax $)$ & $0.0107 \pm 0.0009$ & $0.0097 \pm 0.0013$ & $8.34 \pm 0.10$ \\
log $\left(g\left[\mathrm{~cm} \mathrm{~s}^{-2}\right]\right)$ (inferred) & $8.27 \pm 0.07$ & $8.35 \pm 0.12$ & This study \\
HD 27483 & Böhm-Vitense & Burleigh et al. & $\left(\pi_{\text {sec,Hip }}\right)$ \\
HIP 20284 & $\left(1993 ; \pi_{\text {sec,Schwan }(1991)}\right)$ & $\left(1998 ; \pi_{\text {Hip }}\right)$ & $21555 \pm 84$ \\
$T_{\text {eff }}[\mathrm{K}]$ & 23800 & $21815 \pm 178$ & $8.11 \pm 0.07$ \\
$\log \left(g\left[\mathrm{~cm} \mathrm{~s}^{-2}\right]\right)$ & 8.03 & $8.34 \pm 0.16$ & $0.696 \pm 0.041$ \\
$M / M_{\odot}$ & 0.60 & $0.84 \pm 0.10$ & $0.0121 \pm 0.0006$ \\
$R / R_{\odot}$ & 0.012 & $0.010 \pm 0.002$ & \\
\hline \hline
\end{tabular}

Table 5). Unfortunately, the relatively large uncertainty in the best-fit $\log g$ values prevents an accurate mass determination. It has therefore been common practice to infer the surface gravity of the white dwarf from its astrometric mass, obtained from the orbital elements of the binary, and its radius, obtained from its observed and modelled flux combined with its Hipparcos parallax (Table 5).

A more precise estimate of the white dwarf radius is available through its secular parallax. The latter is non-suspect $\left(g_{\text {Hip }}=0.06\right)$, and fully consistent with the Hipparcos parallax $\left(\pi_{\text {Hip }}=21.37 \pm 1.62\right.$ mas; $\pi_{\text {sec,Hip }}=$ $21.00 \pm 0.40$ mas). The long time-baseline Tycho-2 secular parallax, which might be preferred over the Hipparcos secular parallax in view of the binary nature of the system (although $P_{\text {orb }}=0.521$ days only; Stefanik \& Latham 1992; Sect. 4.3), places the object at a slightly, though not significantly, larger distance $\left(\pi_{\text {sec, Tycho-2 }}=20.56 \pm 0.33\right.$ mas with $g_{\text {Tycho-2 }}=0.02$ ). The Hipparcos secular parallax fits Werner \& Rauch's (1997) model for a radius of $R / R_{\odot}=0.0098 \pm 0.0011$; the corresponding surface gravity is $\log g=8.34 \pm 0.10$ for $M / M_{\odot}=0.759 \pm 0.020$ (Table 5). These values are fully consistent with but (in principle) more precise than the results of Werner \& Rauch (1997) and Barstow et al. (1997).

HD 27483: In 1993, Böhm-Vitense reported the serendipitous discovery of a DA white dwarf companion around the close F6V-plus-F6V binary HD 27483 (HIP 20284). Böhm-Vitense interpreted her spectra using Wesemael et al.'s (1980) unblanketed white dwarf models and
Hamada \& Salpeter's (1961) mass-radius relation, assuming a distance to the system of $47.6 \mathrm{pc}$ (the secular parallax derived by Schwan 1991; Table 5). Recently, Burleigh et al. (1998) presented an analysis of the object, based on updated atmosphere and evolutionary models (Koester 1991; Wood 1995), using its Hipparcos paral$\operatorname{lax}\left(\pi_{\text {Hip }}=21.80 \pm 0.85\right.$ mas $)$. The orbital motion of the binary $\left(P_{\text {orb }}=3.05\right.$ days; Mayor \& Mazeh 1987) has not hampered the Hipparcos measurements: its secular parallax $\left(\pi_{\mathrm{sec}, \mathrm{Hip}}=20.59 \pm 0.35 \mathrm{mas}\right)$ is well defined $\left(g_{\text {Hip }}=2.73\right)$. We use cubic spline interpolation in Burleigh's Table 5 to derive $\log g, T_{\text {eff }}, M$, and $R$ for both the Hipparcos trigonometric and secular parallaxes (Table 5). The secular parallax-based white dwarf mass $\left(M / M_{\odot}=0.70 \pm 0.04\right)$ is significantly smaller than Burleigh et al.'s value $\left(M / M_{\odot}=0.94\right)$. The new mass estimate resolves the problem (acknowledged by Burleigh et al.) that the sum of the cooling age of a $M=0.94 M_{\odot}$ white dwarf and the evolutionary age of its progenitor is significantly shorter than the nuclear age of the Hyades.

\section{Summary and discussion}

At $\sim 45$ pc, the Hyades is the nearest open cluster to the Sun. Its tidal radius of $\sim 10 \mathrm{pc}$ translates to an angular extent of several tens of degrees on the sky and to a significant line-of-sight extension compared to its mean distance. Hipparcos trigonometric parallaxes, with typical accuracies of $\sim 1.5$ mas, constrain the positions of members in the cluster to within a few parsec. Although this precision is sufficient to study the three-dimensional structure of the cluster, and related issues like mass segregation. 
uncertainties in the "trigonometric distances" to individual stars dominate the error budget when constructing the colour-absolute magnitude diagram of the cluster. The considerable tangential velocity of the cluster (relative to the Sun) opens the possibility to derive parallaxes for individual stars from their measured proper motions (Sect. 5). Despite the presence of an internal velocity dispersion in the cluster of $\sim 0.30 \mathrm{~km} \mathrm{~s}^{-1}$ (in one dimension), the proper motion-based (secular) parallaxes are $\sim 3$ times more precise than the Hipparcos trigonometric values (Sect. 5.4).

The secular parallaxes are derived under the assumption that all cluster stars follow a three-dimensional Gaussian velocity distribution with an isotropic dispersion (Sect. 3). A careful analysis confirms the absence of significant velocity structure in the form of expansion, rotation, or shear (Sect. 6). Numerical and theoretical work suggests that members beyond the tidal radius of the cluster (the halo and moving group population) (may) have a systematically different velocity field from the main body of the cluster (the core and corona). We show that the maximum expected systematic secular parallax errors in the outer regions of the cluster are $\lesssim 0.30$ mas (Sect. 6.5), i.e., a factor $\sim 2$ smaller than typical random secular parallax errors. The Hipparcos trigonometric and secular parallaxes as a set are statistically fully consistent. Nonetheless, we do find evidence for the presence of spatially correlated Hipparcos measurements on small angular scales (i.e., a few degrees), consistent with the predictions of the Hipparcos data reduction consortia (Sect. 6.2). The maximum "amplitude" of the correlation is $\lesssim 0.50-0.75 \sigma \sim 0.75-1.00$ mas per star, which is a factor of $\sim 2$ smaller than the value quoted by Narayanan \& Gould (1999b).

Our list of Hyades candidate members contains 233 stars (Sect. 4). These are the 218 candidates selected by P98 and 15 new Hipparcos stars selected by de Bruijne's (1999a) and/or Hoogerwerf \& Aguilar's (1999) methods. Only one of the latter stars (HIP 19757) is a likely member (Sect. 5.2). Long time-baseline Tycho-2 proper motions are available for (most of) the brighter Hyades that were observed by Hipparcos; the corresponding secular parallaxes are fully consistent with both the Hipparcos trigonometric and the Hipparcos secular parallaxes.

The secular parallaxes for members of the Hyades allow the construction of the most precise colour-absolute magnitude diagram of the cluster to date (Figs. 10 and 13; see also, e.g., Madsen 1999). The main sequence is well defined, and shows a few conspicuous but artificial gaps, e.g., around $(B-V)=0.95$ and between $(B-V)=0.30$ and $(B-V)=0.35 \mathrm{mag}$, caused by the suppression of (double and multiple) stars from our sample (cf. Fig. 21 of P98). The small gap between $(B-V)=0.75$ and 0.80 mag is also present in the original sample and is therefore probably real (cf. Fig. 2 of Mermilliod 1981). Somewhat further to the blue, there are two conspicuous features ("gaps" and/or "turn-offs") around $(B-V) \sim 0.30\left(T_{\text {eff }} \sim 7000 \mathrm{~K}\right)$ and $\sim 0.45 \mathrm{mag}(\sim 6400 \mathrm{~K})$. These features, which have never been observed this clearly before, but the existence of which was predicted by E. Böhm-Vitense already 30 years ago, are related to the use of the $(B-V)$ colour as temperature indicator. We suspect, following BöhmVitense, that sudden changes in the properties of surface convection zones in the atmospheres of stars with $(B-V) \sim 0.30$ and $\sim 0.45 \mathrm{mag}$ significantly affect the emergent UV and blue-optical fluxes, and thus the $(U-B)$ and $(B-V)$ colours (Sect. 10.2).

As the Hipparcos members of the Hyades span a large range in mass and occupy a number of different evolutionary states, their effective temperatures and luminosities provide stringent constraints on both the global characteristics of the Hyades (such as metallicity, Helium content, and age) and stellar evolutionary modelling in general (cf. Lebreton 2000). We combine the secular parallaxes derived in this study with two existing $(B-V) \longrightarrow \log T_{\text {eff }}$ and $M_{V} \longrightarrow \log \left(L / L_{\odot}\right)$ calibrations (Sect. 9.4) to infer the fundamental properties of the cluster as well as of a variety of members. The latter include, among others, stars with surface convection zones, Am stars, a $\delta$ Scuti pulsator, red giants, and white dwarfs (Sect. 10). We show that neither the Bessell et al. (1998) nor the Lejeune et al. (1998) $(B-V)-T_{\text {eff }}$ and $T_{\text {eff }}-\mathrm{BC}_{V}$ calibrations (whether correcting for the non-Solar metallicity of the Hyades or not) are appropriate throughout the entire mass range studied in this paper; only an ad-hoc combination of the two calibrations provides an acceptable fit to the P98 CESAM isochrones.

An optimum exploitation of the secular parallax data requires several future steps. An obvious need is the construction of a set of isochrones that are based on homogeneous stellar evolutionary modelling, tailored to the Hyades cluster in terms of chemical composition and age, from the low-mass main sequence $\left(M \gtrsim 0.50 M_{\odot}\right)$ through the clump-giant region (Sect. 10.4). The lack of this information prevents us from validating the chemical composition and age of the cluster derived by P98. The precision with which the secular parallaxes constrain the locations of stars in the HR diagram brings out such detailed structure in the main sequence (e.g., the BöhmVitense gaps) that the construction of future isochrones will require a fine-tuned mass sampling as well as, for stars near the Böhm-Vitense gap, inclusion of stellar rotation (Sect. 10.2). This study has shown that the most significant uncertainty in the secular parallax-based HR diagram locations of stars is now set by systematic errors in the available transformations of the observed optical broad-band colours and absolute magnitudes to effective temperatures and luminosities (cf. Nordström et al. 1997; Castellani et al. 2001). This issue clearly requires future study, for which the secular parallaxes provide stringent boundary conditions.

An application of the secular parallax method to other nearby clusters, such as Coma Berenices, the Pleiades, and Praesepe, is feasible. Improved parallaxes can be obtained for these groups (cf. Dravins et al. 1999), although several complicating factors exist (as compared to the Hyades). Among these are their larger mean 
distances, smaller fields on the sky, less well-defined (Hipparcos) membership lists, and the presence of interstellar reddening and extinction. The secular parallaxes for the Hyades cluster presented in this paper will only be superseded by the measurements of a second generation of astrometric satellites, such as FAME, DIVA, and notably GAIA (http://aa.usno.navy.mil/fame/, wWw.aip.de/groups/DIVA/, astro.estec.esa.nl/GAIA/).

Acknowledgements. The authors wish to thank warmly Adriaan Blaauw for his continuous interest in and impetus on this project. Stimulating discussions with Francesca d'Antona, Anthony Brown, Jan Lub, and Michael Perryman, as well as the constructive criticism of the referees, Lennart Lindegren and Yveline Lebreton, are gratefully acknowledged. The Hipparcos Hyades team (P98) kindly provided BDA Tycho membership information, CESAM isochrones, and stellar masses. This research is based to a large extent on data obtained by ESA's Hipparcos satellite, and has made use of the ADS (NASA) and SIMBAD (CDS) services; it is partly supported by NWO.

\section{Appendix A: Data}

Tables A.1-A.2 contain data for the 218 Hyades candidates selected by P98 (Sect. 4.1) and the 15 new candidates selected by de Bruijne (1999a) and Hoogerwerf \& Aguilar (1999; Sect. 4.2), respectively. The fundamental stellar parameters listed in Cols. (10-13) of Table A.1 are based on the Hipparcos secular parallaxes, and the $V$ and $(B-V)$ values listed in the Hipparcos Catalogue (ESA 1997; fields H5 and H37, respectively; cf. Sect. 9.4).

Table A.1: The 218 P98 Hyades candidates. Columns: (1) Hipparcos number; (2) Tycho identifier; (3) Hipparcos trigonometric parallax $\pi_{\text {Hip }}$ and associated error (mas); (4) Hipparcos secular parallax $\pi_{\text {sec,Hip }}$ and error (mas); (5) Hipparcos goodness-of-fit parameter $g_{\text {Hip }}$; (6) Tycho-2 secular parallax $\pi_{\text {sec,Tycho-2 }}$ and error (mas); (7) Tycho-2 goodness-of-fit parameter $g_{\text {Tycho-2 }}$; (8) mass $M$ (in $M_{\odot}$; from Sect. 5.3 in P98; cf. Sect. 9.4.5); (9) logarithm of effective temperature $T_{\text {eff }}$ (in $\mathrm{K}$ ) and associated error; (10) bolometric correction $\mathrm{BC}_{V}$ and error (mag); (11) logarithm of luminosity $\log \left(L / L_{\odot}\right)$ and error; (12) logarithm of surface gravity $\log \left(g\left[\mathrm{~cm} \mathrm{~s}^{-2}\right]\right)$ and error; (13) miscellaneous flags: (a) "1" if an optical companion was not counted in the mass; (b) "1" if the mass is particularly uncertain (from P98); (c) "1" = spectroscopic binary ("SB"), "2" = radial velocity variable ("RV") (cf. Col. (s) in P98's Table 2); (d) Hipparcos duplicity flag H56 ("I" = 1, "M" = 2, "H" = 3; cf. Col. (t) in P98's Table 2); (e) Hipparcos duplicity flags H59 and H61 ("C" = 1, "G" = 2 , "O" = 3, "V" = 4, "X" = 5, "S" = 6, "X/S" = 7; cf. Col. (u) in P98's Table 2); (f) Hipparcos photometric variability flag H52 ("C" or "empty" = 0, "D" = 1, "M" = 2, "P" = 3, "R" = 4, "U" = 5); (g) "1" or "2" if Cols. (10-13) were derived using calibration (1) or (2) (Sects. 9.4.2 and 9.4.3); (h) P98 membership (Col. (x) in their Table 2; "1" if based on proper motion and radial velocity, " 2 " if based on proper motion only, "3" / 6" if " 1 " / 2 " but rejected by de Bruijne (1999a), "4"/ "7" if " 1 "/ 2 " but rejected by Hoogerwerf \& Aguilar (1999), "5" / "8" if "1" / "2" but rejected by de Bruijne and Hoogerwerf \& Aguilar); (i) index to notes (" 1 " if note is present). This table is only available in electronic form at the CDS via anonymous ftp to cdsarc.u-strasbg.fr (130.79.128.5) or via http://cdsweb.u-strasbg.fr/cgi-bin/qcat?J/A+A/367/111

\section{Appendix B: $(B-V)-M_{V} \longrightarrow \log T_{\text {eff }}-$ $\log \left(L / L_{\odot}\right)$}

\section{B.1. The giants $\epsilon$ and $\gamma$ Tau}

The Hyades cluster contains two single giants, $\epsilon$ Tau (HIP 20889) and $\gamma$ Tau $^{21}$ (HIP 20205; Sects. 9.3 and 10.4). Both stars are located in the giant clump, i.e., the core Helium burning phase. Table B. $1^{22}$ shows the results of applying calibration (1) (Sect. 9.4.2) to $\epsilon$ and $\gamma$ Tau for $[\mathrm{Fe} / \mathrm{H}]=0$ and +0.14 (although it formally applies to dwarfs only).

The effective temperatures, surface gravities, metallicities, and bolometric fluxes of $\epsilon$ and $\gamma$ Tau have recently been determined through combining the infra-red flux method with modelling of high-resolution spectra using MARCS model atmospheres (Table B.1; Smith \& Ruck 1997; Smith 1999). Luminosities for both stars can be obtained by combining their bolometric fluxes with parallaxes; results for Hipparcos trigonometric and secular parallaxes are listed in Table B.1 under Smith \& Ruck (1997) and Smith (1999), respectively. Although calibration (1) gives very similar results, we use the infra-red flux method temperatures and secular parallax-based luminosities.

\section{B.2. The giants $\delta^{1}$ and $\theta^{1}$ Tau and $\delta$ Ari}

The giant $\delta^{1}$ Tau (HIP 20455) forms a common-propermotion pair with the turn-off-region object $\delta^{2}$ Tau (HIP 20542) at a separation of $\sim 0 \% 05$. The giant itself is a single-lined spectroscopic binary (Griffin \& Gunn $1977 ; P=530$ days, $K_{1}=3 \mathrm{~km} \mathrm{~s}^{-1} ; 1=\mathrm{A}=$ primary; $2=\mathrm{B}=$ secondary); speckle observations have allowed the detection of a companion at $0^{\prime \prime} .273$ (Mason et al. 1993; but see Patience et al. 1998). Despite the duplicity of $\delta^{1}$ Tau, its secular parallax solution is well defined $\left(\pi_{\mathrm{sec}, \mathrm{Hip}}=21.16 \pm 0.37\right.$ mas with $g_{\mathrm{Hip}}=0.07$ and

\footnotetext{
${ }^{21}$ Although $\gamma$ Tau was reported as $\rho \sim 0$ "' 395 speckle double by Morgan et al. (1982), Mason et al. (1993) and Patience et al. (1998) could not confirm this result. Griffin \& Holweger (1989) summarize the confusing literature on (non-existent) radial velocity variability, and conclude the object is single.

${ }^{22}$ Although Bessell et al. do provide separate relations for red giants in their Table 5 , the modeled $(B-V)$ range is inconsistent with the measured values for both stars; the same holds for Lejeune's giant calibration in their Tables 1 and 9 .
} 
Table A.2. The 15 new Hyades candidate members (Sect. 4.2) in (extended) P98 Table 2 format, but excluding Cols. (b)-(m). Only HIP 19757 is a likely new member (Sect. 5.2). Columns (cf. P98): (a) Hipparcos and Tycho identifier; (n, o) Hipparcos parallax and error (mas); (p, q) radial velocity and error (km $\mathrm{s}^{-1}$ ); (r) source of radial velocity ("S" means data from SIMBAD [http://cdsweb.u-strasbg.fr/Simbad.html]); (s) "RV" = radial velocity variable (from Duflot et al. 1995); (t) "HIM" = star was previously known, or classified by Hipparcos, to have resolved components (from field H56); (u) "CGOVX" = relevant part of the Hipparcos Double and Multiple Systems Annex (DMSA; field H59) supplemented by "S" = suspected Hipparcos binary (field H61); (v) three-dimensional distance from the cluster center of mass defined by 180 stars (Table 3 in P98; in pc), based on Hipparcos trigonometric parallax; (w) kinematic P98 membership statistic $c$ (their Eq. (16)); (x) final membership assigned by P98 ("0" = non-member, "1" = member); (y) Hoogerwerf \& Aguilar (1999) membership "probability" $p_{\mathrm{HA}}$ (denoted $S$ in their paper), based on proper motion and parallax data (their Sect. 2.4); (z) de Bruijne (1999a) membership probability $p_{\mathrm{dB}}$, based on proper motion data only (his Sect. 2.4.4). The last four columns provide the Hipparcos and Tycho-2 secular parallaxes $\pi_{\text {sec,Hip/Tycho-2 }}$ and corresponding goodness-of-fit parameters $g_{\mathrm{Hip} / \text { Tycho-2 }}$ (Sect. 5). This table is also available in electronic form at the CDS via anonymous ftp to cdsarc.u-strasbg.fr (130.79.128.5) or via http://cdsweb.u-strasbg.fr/cgi-bin/qcat?J/A+A/367/111

\begin{tabular}{|c|c|c|c|c|c|c|c|c|c|c|c|c|}
\hline \multirow{3}{*}{$\begin{array}{c}\text { HIP \& TYC } \\
\text { (a) }\end{array}$} & \multirow{3}{*}{$\begin{array}{c}\text { Parallax } \\
\pi_{\text {Hip }} \pm \sigma_{\pi, \mathrm{Hip}} \\
(\mathrm{n}, \mathrm{o}) \\
\end{array}$} & \multirow{3}{*}{$\begin{array}{l}\text { Radial velocity } \\
v_{\text {rad }} \pm \sigma_{v_{\text {rad }}} \\
\quad(\mathrm{p}, \mathrm{q}, \mathrm{r})\end{array}$} & \multirow{3}{*}{$\begin{array}{l}\text { Multi- } \\
\text { plicity } \\
(\mathrm{s}, \mathrm{t}, \mathrm{u})\end{array}$} & \multicolumn{5}{|c|}{ Membership } & \multicolumn{4}{|c|}{ Secular parallaxes } \\
\hline & & & & \multirow[b]{2}{*}{$(\mathrm{v})$} & \multirow{2}{*}{$\begin{array}{c}c \\
(\mathrm{w})\end{array}$} & \multirow[b]{2}{*}{$(\mathrm{x})$} & \multirow{2}{*}{$\begin{array}{c}p_{\mathrm{HA}} \\
(\mathrm{y})\end{array}$} & \multirow{2}{*}{$\begin{array}{c}p_{\mathrm{dB}} \\
(\mathrm{z})\end{array}$} & \multicolumn{2}{|c|}{ Hipparcos } & \multicolumn{2}{|c|}{ Tycho-2 } \\
\hline & & & & & & & & & $\pi_{\text {sec,Hip }}$ & $g_{\text {Hip }}$ & $\pi_{\mathrm{sec}, \text { Tycho-2 }}$ & $g_{\text {Tycho- }-2}$ \\
\hline $14232=1794018181$ & $20.01 \pm 0.88$ & $+10.80 \pm 2.00(\mathrm{~S})$ & & 19.20 & & & & 0.76 & $9.42 \pm 0.23$ & 181.88 & $8.71 \pm 0.28$ & 0.04 \\
\hline $18965=1826$ & 28.44 & -10.30 & & 15.17 & & & & & $41.59 \pm 0.28$ & 170 & $48 \pm 0.32$ & 7.09 \\
\hline $19757=0671$ & 16.56 & & & 15.40 & & & 0.10 & 0 & $20.19 \pm 1.04$ & 2.67 & $2 \pm 0.71$ & 5.26 \\
\hline $19981=1815$ & 30.56 & $-28,8$ & $\mathrm{~B}_{\mathrm{N}}$ & 14.80 & 2 & 0 & & & 20 & & 39 & 0.04 \\
\hline $20616=0$ & $21.00 \pm$ & & & 3.91 & & & & 0.06 & $9 \pm 0.42$ & 137.63 & $9.56 \pm 0.42$ & 76.52 \\
\hline $20693=0081016291$ & $22.03 \pm 0.90$ & $+29.67 \pm 0.30(1)$ & & 9.31 & 17.54 & 0 & 0.19 & 0.55 & $19.67 \pm 0.37$ & 13.47 & $18.98 \pm 0.47$ & 5.29 \\
\hline $20777=1$ & 25.7 & & V & 10.45 & & & & & & 23.77 & & \\
\hline $20895=1$ & 25.0 & $-16.00 \pm 9.99(\mathrm{~S})$ & $\mathrm{HC}$ & 9.9 & & & & 0. & 14.38 & 11.21 & $15.60 \pm 0.61$ & 11.19 \\
\hline $20904=2372021011^{\mathrm{b}}$ & & $-36.60 \pm 2.00(\mathrm{~S})$ & RV I C & 14. & & & & 0 & & & & 18.02 \\
\hline $21475=0690007971$ & $18.93 \pm$ & & I & 7.50 & 20.92 & 0 & & 0.37 & $34.96 \pm 0.58$ & 105.05 & $36.10 \pm 0.51$ & 6.29 \\
\hline $21760=1842012641$ & $13.17 \pm 0.94$ & $+24.80 \pm 2.00(\mathrm{~S})$ & & 32.39 & & & 0.22 & & $11.55 \pm 0.35$ & 3.77 & $12.28 \pm 0.36$ & 0.04 \\
\hline $21961=1830021271$ & $20.03 \pm 0.71$ & $+7.70 \pm 2.00(\mathrm{~S})$ & & 7.70 & & & & 0.28 & $5.50 \pm 0.40$ & 556.20 & $4.67 \pm 0.32$ & 131.19 \\
\hline $22449=0096014621$ & $124.60 \pm 0.95$ & $+24.10 \pm 0.90(\mathrm{~S})$ & RV I & 38.99 & & & 0.6 & & $122.54 \pm 0.48$ & 26.42 & $113.44 \pm 0.38$ & 0.04 \\
\hline $24480=1291003851$ & $16.54 \pm 1$ & +21.70 & G & 17.51 & & & 0.15 & 0.21 & 19. & 10.38 & \pm 0.48 & 27.80 \\
\hline $25730=1860006281^{\mathrm{c}}$ & $11.24 \pm 0.84$ & $+13.20 \pm 2.00(\mathrm{~S})$ & & 47.24 & & & 0.25 & & $12.71 \pm 0.39$ & 8.60 & $12.98 \pm 0.41$ & 17.69 \\
\hline
\end{tabular}

${ }^{a}$ : T Tauri-type star with two components (SIMBAD); Tycho-1 number suppressed in Tycho-2 catalogue.

${ }^{\mathrm{b}}$ : A component in double system (SIMBAD).

${ }^{\mathrm{c}}$ : T Tauri-type star (SIMBAD). 
Table B.1. Fundamental properties of the single Hyades red giants (cf. Table 2 in Smith \& Ruck 1997). The luminosities $\log \left(L / L_{\odot}\right)$ listed for Smith \& Ruck (1997) and Smith (1999) were derived from combining integrated stellar fluxes above the Earth's atmosphere determined using the infra-red flux method $\left(F_{\gamma}\right.$ Tau $=1.1410^{-9} \mathrm{~W} \mathrm{~m}^{-2} ; F_{\epsilon} \mathrm{Tau}=1.2810^{-9} \mathrm{~W} \mathrm{~m}^{-2}$; Blackwell \& Lynas-Gray 1998) with Hipparcos trigonometric and secular parallaxes, respectively. The giant metallicities $[\mathrm{Fe} / \mathrm{H}]=+0.14$ and masses $M / M_{\odot}=2.32 \pm 0.10$ used in calibration (1) were taken from P98. These mass estimates are consistent with the clump giant masses of $\sim 2.4 M_{\odot}$ listed by Torres et al. (1997a,c)

\begin{tabular}{|c|c|c|c|c|}
\hline $\begin{array}{l}\gamma \text { Tau (K0III) } \\
\text { HIP } 20205\end{array}$ & $\begin{array}{r}\text { Smith \& Ruck }(1997) \\
L=L\left(\pi_{\mathrm{Hip}}\right)\end{array}$ & $\begin{array}{r}\text { Smith (1999) } \\
L=L\left(\pi_{\text {sec }, \text { Hip }}\right)\end{array}$ & $\begin{array}{l}\text { Calibration (1) } \\
{[\mathrm{Fe} / \mathrm{H}]=+0.14}\end{array}$ & $\begin{array}{r}\text { Calibration (1) } \\
{[\mathrm{Fe} / \mathrm{H}]=0}\end{array}$ \\
\hline $\begin{array}{l}\log \left(L / L_{\odot}\right) \\
\log \left(T_{\text {eff }}[\mathrm{K}]\right)^{\mathrm{a}} \\
\log \left(g\left[\mathrm{~cm} \mathrm{~s}^{-2}\right]\right) \\
{[\mathrm{Fe} / \mathrm{H}]^{\mathrm{c}}} \\
M / M_{\odot}\end{array}$ & $\begin{array}{r}1.901 \pm 0.016 \\
3.6959 \pm 0.0035 \\
2.65 \pm 0.20 \\
+0.12 \pm 0.03\end{array}$ & $\begin{array}{r}1.869 \pm 0.016 \\
\\
2.63 \pm 0.03 \\
+0.150 \pm 0.029 \\
2.30 \pm 0.10\end{array}$ & $\begin{array}{r}1.843 \pm 0.016 \\
3.7004 \pm 0.0027 \\
2.714 \pm 0.027 \\
+0.14 \\
2.32 \pm 0.10\end{array}$ & $\begin{array}{r}1.850 \pm 0.016 \\
3.6967 \pm 0.0027 \\
2.692 \pm 0.027 \\
+0.00 \\
2.32 \pm 0.10\end{array}$ \\
\hline \multicolumn{5}{|l|}{$\begin{array}{l}\epsilon \text { Tau (K0III) } \\
\text { HIP } 20889\end{array}$} \\
\hline $\begin{array}{l}\log \left(L / L_{\odot}\right) \\
\log \left(T_{\text {eff }}[\mathrm{K}]\right)^{\mathrm{b}} \\
\log \left(g\left[\mathrm{~cm} \mathrm{~s}^{-2}\right]\right) \\
{[\mathrm{Fe} / \mathrm{H}]^{\mathrm{c}}} \\
M / M_{\odot}\end{array}$ & $\begin{array}{r}1.956 \pm 0.015 \\
3.6912 \pm 0.0031 \\
2.45 \pm 0.20 \\
+0.15 \pm 0.03\end{array}$ & $\begin{array}{r}1.927 \pm 0.015 \\
\\
2.57 \pm 0.03 \\
+0.163 \pm 0.030 \\
2.30 \pm 0.10 \\
\end{array}$ & $\begin{array}{r}1.912 \pm 0.015 \\
3.6938 \pm 0.0022 \\
2.619 \pm 0.026 \\
+0.14 \\
2.32 \pm 0.10\end{array}$ & $\begin{array}{r}1.919 \pm 0.015 \\
3.6902 \pm 0.0022 \\
2.598 \pm 0.026 \\
+0.00 \\
2.32 \pm 0.10 \\
\end{array}$ \\
\hline$T_{\text {eff }, \gamma}$ Tau $-\epsilon$ Tau $[\mathrm{K}]$ & $+54 \pm 53$ & $+80 \pm 20$ & $+76 \pm 40$ & $+74 \pm 40$ \\
\hline
\end{tabular}

${ }^{\mathrm{a}}: \log \left(T_{\mathrm{eff}}[\mathrm{K}]\right)=3.6955 \pm 0.0013($ Taylor 1999).

${ }^{\mathrm{b}}: \log \left(T_{\text {eff }}[\mathrm{K}]\right)=3.6985 \pm 0.0013$ (Taylor 1999).

${ }^{\mathrm{c}}:[\mathrm{Fe} / \mathrm{H}]=+0.104 \pm 0.009$ (Taylor 1999).

Table B.2. Fundamental parameters of the primary components of $\delta^{1}$ and $\theta^{1}$ Tau and $\delta$ Ari. The McWilliam temperature errors have been chosen as 0.0025. Taylor's (1999) catalogue lists (homogeneously determined) mean metallicities and temperatures based on literature results. The giant masses and metallicities used in calibration (1) were taken from P98

\begin{tabular}{|c|c|c|c|c|}
\hline $\begin{array}{l}\delta^{1} \text { Tau A (K0III) } \\
\text { HIP } 20455\end{array}$ & $\begin{array}{r}\text { Blackwell \& } \\
\text { Lynas-Gray (1998) }\end{array}$ & "McWilliam (1990) & Taylor (1999) & $\begin{array}{l}\text { Calibration (1) } \\
{[\mathrm{Fe} / \mathrm{H}]=+0.14}\end{array}$ \\
\hline $\begin{array}{l}\log \left(L / L_{\odot}\right) \\
\log \left(T_{\text {eff }}[\mathrm{K}]\right) \\
\log \left(g\left[\mathrm{~cm} \mathrm{~s}^{-2}\right]\right) \\
{[\mathrm{Fe} / \mathrm{H}]} \\
M / M_{\odot}\end{array}$ & $3.6927 \pm 0.0039$ & $\begin{array}{r}3.6937 \pm 0.0025 \\
2.85 \\
+0.00 \pm 0.07\end{array}$ & $\begin{array}{l}3.6973 \pm 0.0013 \\
+0.104 \pm 0.009\end{array}$ & $\begin{array}{r}1.835 \pm 0.016 \\
3.6999 \pm 0.0022 \\
2.716 \pm 0.026 \\
+0.14 \\
2.30 \pm 0.10\end{array}$ \\
\hline $\begin{array}{l}\theta^{1} \text { Tau A (K0III) } \\
\text { HIP } 20885\end{array}$ & $\begin{array}{r}\text { Torres et al. } \\
(1997 \mathrm{c})\end{array}$ & & & \\
\hline $\begin{array}{l}\log \left(L / L_{\odot}\right) \\
\log \left(T_{\text {eff }}[\mathrm{K}]\right)^{\mathrm{a}} \\
\log \left(g\left[\mathrm{~cm} \mathrm{~s}^{-2}\right]\right) \\
{[\mathrm{Fe} / \mathrm{H}]} \\
M / M_{\odot}\end{array}$ & $\begin{array}{r}1.98 \\
3.6902 \pm 0.0089 \\
2.63 \pm 0.07 \\
2.91 \pm 0.88\end{array}$ & $\begin{array}{r}3.6955 \pm 0.0025 \\
3.17 \\
+0.04 \pm 0.10\end{array}$ & $\begin{array}{l}3.7042 \pm 0.0013 \\
+0.104 \pm 0.009\end{array}$ & $\begin{array}{r}1.791 \pm 0.016 \\
3.7062 \pm 0.0022 \\
2.789 \pm 0.026 \\
+0.14 \\
2.32 \pm 0.10\end{array}$ \\
\hline $\begin{array}{l}\delta \text { Ari (K2IIIvar) } \\
\text { HIP } 14838\end{array}$ & $\begin{array}{r}\text { Calibration (1) } \\
{[\mathrm{Fe} / \mathrm{H}]=0}\end{array}$ & & & \\
\hline $\begin{array}{l}\log \left(L / L_{\odot}\right) \\
\log \left(T_{\text {eff }}[\mathrm{K}]\right) \\
\log \left(g\left[\mathrm{~cm} \mathrm{~s}^{-2}\right]\right) \\
{[\mathrm{Fe} / \mathrm{H}]} \\
M / M_{\odot}\end{array}$ & $\begin{array}{r}1.673 \pm 0.013 \\
3.6837 \pm 0.0023 \\
2.815 \pm 0.025 \\
+0.00 \\
2.31 \pm 0.10\end{array}$ & $\begin{array}{r}3.6821 \pm 0.0025 \\
2.93 \\
-0.03 \pm 0.09\end{array}$ & $\begin{array}{l}3.6817 \pm 0.0025 \\
-0.012 \pm 0.049\end{array}$ & $\begin{array}{r}1.665 \pm 0.013 \\
3.6872 \pm 0.0024 \\
2.837 \pm 0.025 \\
+0.14 \\
2.31 \pm 0.10\end{array}$ \\
\hline
\end{tabular}

${ }^{\mathrm{a}}: \log \left(T_{\text {eff }}[\mathrm{K}]\right)=3.6803$ (Ridgway et al. 1980) and $\log \left(T_{\text {eff }}[\mathrm{K}]\right)=3.6665 \pm 0.0075$ (Peterson et al. 1981).

$\pi_{\text {sec }, \text { Tycho-2 }}=21.14 \pm 0.28$ mas with $g_{\text {Tycho-2 }}=0.36$; cf. $\left.\pi_{\mathrm{Hip}}=21.29 \pm 0.93 \mathrm{mas}\right)$. Table B.2 provides $\log T_{\mathrm{eff}}$, $\log \left(L / L_{\odot}\right)$, and $\log g$ for the primary component using calibration (1) (Sect. 9.4.2) for $[\mathrm{Fe} / \mathrm{H}]=+0.14$.
The giant $\theta^{1}$ Tau (HIP 20885) forms a commonproper-motion pair with the spectroscopic binary $\theta^{2}$ Tau (Appendix B.3) at a separation of $\sim 0.10\left(\theta^{1}\right.$ and $\theta^{2}$ Tau were independently observed by Hipparcos as "single 
Table B.3. Fundamental parameters of the components of the spectroscopic binary $\theta^{2}$ Tau (HIP 20894; cf. Table 3). The symbol $q \equiv M_{2} / M_{1} \leq 1$ denotes the mass ratio of the two components $(1=\mathrm{A}=$ primary; $2=\mathrm{B}=$ secondary). Peterson et al. (1993) based their results on spectral line modelling using Kurucz (1991) models; their component masses are likely underestimated. Królikowska (1992) used stellar evolutionary models lacking convective core overshoot. Her results depend sensitively on the adopted evolutionary status and chemical composition of the primary; values listed here assume a primary in the thick Hydrogen shell burning phase, $(X, Y, Z)=(0.70,0.27,0.03)$, and $\log (\tau / \mathrm{yr}) \sim 8.73\left(\mathrm{cf} . \log (\tau / \mathrm{yr})=8.80_{-0.11}^{+0.05}\right.$ for $Z=0.027_{-0.011}^{+0.023}$ [Lastennet et al. 1999]; $\log (\tau / \mathrm{yr})=8.80 \pm 0.04$ for $Z=0.024 \pm 0.003$ [P98]). A \& PF stands for Antonello \& Pasinetti Fracassini (1998). We adopt calibration (1) for $[\mathrm{Fe} / \mathrm{H}]=+0.14$ using $M_{1} / M_{\odot}=2.37 \pm 0.10$ and $M_{2} / M_{\odot}=1.95 \pm 0.10$ (Lastennet et al. 1999)

\begin{tabular}{|c|c|c|c|c|}
\hline $\begin{array}{l}\theta^{2} \text { Tau A } \\
(\mathrm{A} 7 \mathrm{III}-\mathrm{IV})\end{array}$ & $\begin{array}{r}\text { Peterson et al. } \\
(1993) \\
\end{array}$ & $\begin{array}{r}\text { Królikowska (1992) } \\
+ \text { Breger et al. (1987) }\end{array}$ & $\begin{array}{r}\text { A \& PF + } \\
\text { Tomkin et al. (1995) }\end{array}$ & $\begin{array}{l}\text { Calibration } \\
{[\mathrm{Fe} / \mathrm{H}]=+0.14}\end{array}$ \\
\hline $\begin{array}{l}\log \left(L / L_{\odot}\right) \\
\log \left(T_{\text {eff }}[\mathrm{K}]\right)^{\mathrm{a}} \\
\log \left(g\left[\mathrm{~cm} \mathrm{~s}^{-2}\right]\right) \\
M / M_{\odot}\end{array}$ & $\begin{array}{r}3.9243 \pm 0.0052 \\
3.9 \pm 0.05 \\
1.71 \pm 0.20 \\
\end{array}$ & $\begin{array}{r}1.75 \pm 0.11 \\
3.9191 \pm 0.0052 \\
3.8 \\
2.63 \pm 0.10 \\
\end{array}$ & $\begin{array}{r}1.72 \pm 0.04 \\
3.9138 \pm 0.0053 \\
3.701 \pm 0.058 \\
2.33 \pm 0.21 \\
\end{array}$ & $\begin{array}{r}1.686 \pm 0.016 \\
3.9020 \pm 0.0029 \\
3.687 \pm 0.027 \\
2.37 \pm 0.10 \\
\end{array}$ \\
\hline \multicolumn{5}{|l|}{$\theta^{2}$ Tau B (A5V) } \\
\hline $\begin{array}{l}\log \left(L / L_{\odot}\right) \\
\log \left(T_{\text {eff }}[\mathrm{K}]\right)^{\mathrm{a}} \\
\log \left(g\left[\mathrm{~cm} \mathrm{~s}^{-2}\right]\right) \\
M / M_{\odot}\end{array}$ & $\begin{array}{r}3.9243 \pm 0.0052 \\
4.3 \pm 0.05 \\
1.61 \pm 0.12 \\
\end{array}$ & $\begin{array}{r}1.31 \pm 0.11 \\
3.9243 \pm 0.0052 \\
4.0 \\
2.23 \pm 0.10 \\
\end{array}$ & $1.80 \pm 0.31$ & $\begin{array}{r}1.256 \pm 0.016 \\
3.9154 \pm 0.0031 \\
4.085 \pm 0.030 \\
1.95 \pm 0.10 \\
\end{array}$ \\
\hline $\begin{array}{l}q^{\mathrm{b}} \\
\pi_{\theta^{2} \text { Tau }}[\mathrm{mas}]^{\mathrm{c}} \\
\end{array}$ & $\begin{array}{r}0.94 \pm 0.13 \\
23.58 \pm 0.83 \\
\end{array}$ & $\begin{array}{r}0.85 \pm 0.05 \\
19.72 \pm 1.36 \\
\end{array}$ & $\begin{array}{r}0.76 \pm 0.14 \\
22.68 \pm 1.13 \\
\end{array}$ & $\begin{array}{r}0.82 \pm 0.05 \\
22.24 \pm 0.36 \\
\end{array}$ \\
\hline
\end{tabular}

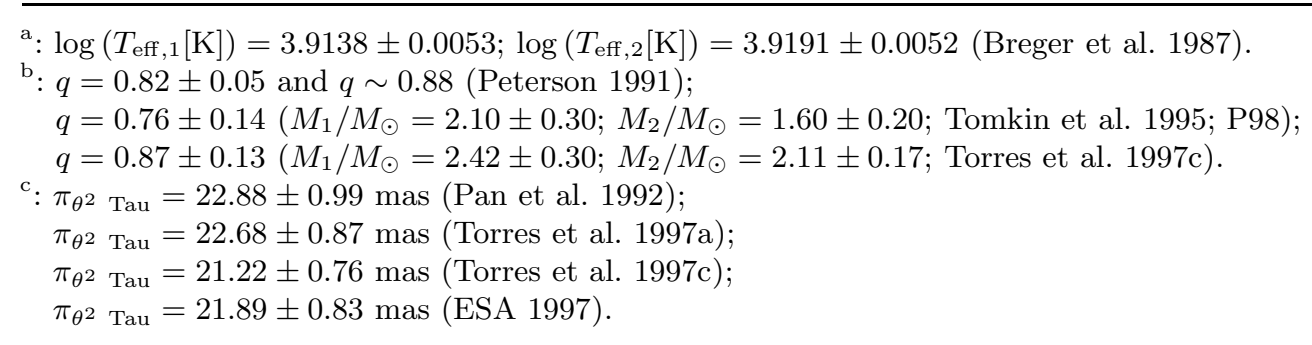

pointings"; field H58 = "1" for both systems). The object $\theta^{1}$ Tau itself is a "resolved single-lined spectroscopic binary" (e.g., Griffin \& Gunn 1977; Torres et al. 1997c; $P=5939$ days; $K_{1}=7.17 \mathrm{~km} \mathrm{~s}^{-1}$ ). Peterson et al. (1981) derived a magnitude difference $\Delta V \sim 3.5 \mathrm{mag}$ by using Lunar occultations; Mason et al. (1993) detected a speckle companion at 0. .048. Torres et al. (1997c) constructed an astrometric-spectroscopic orbital solution for the binary; the fundamental parameters of the primary component were derived by assuming a distance of $47.6 \pm 1.9 \mathrm{pc}$ (following from the orbital parallax of $\theta^{2}$ Tau). This distance, which corresponds to $\pi=21.01 \pm 0.84$ mas, is fully consistent with both the Hipparcos trigonometric parallax $\left(\pi_{\text {Hip }}=20.66 \pm 0.85\right.$ mas $)$ and the Hipparcos secular parallax $\left(\pi_{\mathrm{sec}, \text { Hip }}=21.29 \pm 0.37 \mathrm{mas}\right)$, although the latter is suspect, presumably as a result of duplicity $\left(g_{\text {Hip }}=48.26\right.$; Söderhjelm (1999) lists $\pi_{\text {trigonometric }}=$ $21.3 \pm 1.0$ mas). Fundamental parameters for $\theta^{1}$ Tau A are listed in Table B.2. A comparison with literature values shows that the effective temperatures for both $\delta^{1}$ and $\theta^{1}$ Tau are uncertain; we decided to use the McWilliam (1990) effective temperatures and secular parallax-based luminosities.
The giants $\theta^{1}, \delta^{1}, \epsilon$, and $\gamma$ Tau are all located within $\sim 2.5$ pc of the cluster center (i.e., in the core region). The P98 member list contains one other red giant (HIP 14838; $\delta$ Ari), which is, however, located at $15.34 \mathrm{pc}$ from the cluster center (e.g., Eggen 1983). Although its proper motion, parallax, and radial velocity are consistent with membership ( $c=8.05$ in P98's Table 2), the object was not confirmed as member by de Bruijne (1999a) and Hoogerwerf \& Aguilar (1999) based on its proper motion and parallax (Sect. 4.2; cf. Table A.1). The secular parallax solution derived in Sect. 5 is suspect $\left(g_{\mathrm{Hip}}=66.87\right.$; $\pi_{\text {Hip }}=19.44 \pm 1.23$ and $\pi_{\text {sec,Hip }}=20.28 \pm 0.28$ mas ), which is not surprising if the object is a non-member. However, $\delta$ Ari is a "suspected non-single" star (i.e., Hipparcos field $\mathrm{H} 61=$ "S") and a " $\Delta \mu$ binary" (Wielen et al. 2000), which implies the Hipparcos astrometric parameters, and thus the secular parallax $\pi_{\text {sec,Hip }}$ and corresponding goodnessof-fit parameter $g_{\text {Hip }}$ as well as de Bruijne's conclusion on membership, could be erroneous due to unmodelled orbital motion. The goodness-of-fit parameter of the Tycho2 secular parallax $\left(g_{\text {Tycho-2 }}=74.87\right)$, nonetheless, indicates that the long time-baseline Tycho-2 proper motion is inconsistent with the mean cluster motion. Although the WEB radial velocity catalogue (Duflot et al. 1995) lists the star as single, de Medeiros \& Mayor (1999) 
estimate the "probability that the radial velocity of the star is constant" at 0.494 (based on 2 observations separated by 265 days); no speckle measurements have been reported for $\delta$ Ari. The metallicity of the star has not been determined unambiguously (e.g., $[\mathrm{Fe} / \mathrm{H}]=-0.03 \pm 0.09$ [McWilliam 1990]; $-0.012 \pm 0.049$ [Taylor 1999]), although published values are more consistent with sub-Solar than with the mean value of the Hyades $([\mathrm{Fe} / \mathrm{H}]=+0.14$; Sect. 9.2). Table B.2 lists the parameters of $\delta$ Ari. In view of its near-Solar metallicity, we also applied calibration (1) (Sect. 9.4.2) for $[\mathrm{Fe} / \mathrm{H}]=0$; we use these parameters. The star is likely a non-member (Sect. 10.4).

\section{B.3. The spectroscopic binary $\theta^{2}$ Tau}

Table B.3 summarizes results on the fundamental stellar parameters of the "resolved double-lined spectroscopic binary" $\theta^{2}$ Tau (HIP 20894; Sect. 10.3). The uncertain semiamplitude of the secondary velocity curve translates into a wide range of published mass ratios $q$ (e.g., Peterson 1991, 1993; Torres et al. 1997c). We applied calibration (1) (Sect. 9.4.2) for $[\mathrm{Fe} / \mathrm{H}]=+0.14$ to both components separately (Table B.3). Lunar occultations have provided magnitude and colour differences: $\Delta V \equiv V_{2}-V_{1}=$ $1.10 \pm 0.05$ mag; $\Delta(B-V) \equiv(B-V)_{2}-(B-V)_{1}=$ $-0.006 \pm 0.005 \mathrm{mag}(1=\mathrm{A}=$ primary; $2=\mathrm{B}=$ secondary; Peterson et al. 1981, 1993). The combined magnitude $V=3.40 \pm 0.02 \mathrm{mag}$ and colour $(B-V)=$ $0.179 \pm 0.004 \mathrm{mag}$ therefore yield $V_{1}=3.736 \pm 0.020 \mathrm{mag}$, $V_{2}=4.836 \pm 0.020 \mathrm{mag},(B-V)_{1}=0.170 \pm 0.010 \mathrm{mag}$, and $(B-V)_{2}=0.160 \pm 0.010$ mag. We adopt the calibration (1) values for both components.

\section{References}

Abt, H. A. 1985, ApJ, 294, L103

Abt, H. A., \& Morrell, N. I. 1995, ApJS, 99, 135

Aizenman, M. L., Demarque, P., \& Miller, R. 1969, ApJ, 155, 973

Allende Prieto, C., \& Lambert, D. L. 1999, A\&A, 352, 555

Alonso, A., Arribas, S., \& Martínez-Roger, C. 1996, A\&A, 313, 873

van Altena, W. F., Lee, J. T., \& Hoffleit, E. D. 1995, General catalogue of trigonometric stellar parallaxes; 4th ed. (Yale Univ. Obs., New Haven)

Alves, D. R. 2000, ApJ, 539, 732

Antonello, E., \& Pasinetti Fracassini, L. E. 1998, A\&A, 331, 995

Asiain, R., Figueras, F., \& Torra, J. 1999, A\&A, 350, 434

Babcock, H. W. 1958, ApJ, 128, 228

Balachandran, S. C. 1995, ApJ, 446, 203

Barstow, M. A., Holberg, J. B., Cruise, A. M., \& Penny, A. J. 1997, MNRAS, 290, 505

Barstow, M. A., Schmitt, J. H. M. M., Clemens, J. C., et al. 1992, MNRAS, 255, 369

van den Bergh, S. 1977, ApJL, 215, 103

Bertiau, F. C. 1958, ApJ, 128, 533

Bessell, M. S., Castelli, F., \& Plez, B. 1998, A\&A, 333, 231 (erratum A\&A, 337, 321)
Blaauw, A. 1946, Ph.D. Thesis, Groningen Univ. (Publ. Kapteyn Astron. Lab., 52, 1)

Blackwell, D. E., \& Lynas-Gray, A. E. 1994, A\&A, 282, 899

Blackwell, D. E., \& Lynas-Gray, A. E. 1998, A\&AS, 129, 505

Blackwell, D. E., Lynas-Gray, A. E., \& Petford, A. D. 1991, A\&A, 245, 567

Blackwell, D. E., Petford, A. D., Haddock, D. J., Arribas, S., \& Selby, M. J. 1990, A\&A, 232, 396

Boesgaard, A. M. 1989, ApJ, 336, 798

Boesgaard, A. M., \& Tripicco, M. J. 1986, ApJL, 302, 49

Boesgaard, A. M., \& Friel, E. D. 1990, ApJ, 351, 467

Böhm-Vitense, E. 1953, Z. Astrophys., 32, 135

Böhm-Vitense, E. 1958, Z. Astrophys., 46, 108

Böhm-Vitense, E. 1970, A\&A, 8, 283 \& 299

Böhm-Vitense, E. 1981, ARA\&A, 19, 295

Böhm-Vitense, E. 1982, ApJ, 255, 191

Böhm-Vitense, E. 1993, AJ, 106, 1113

Böhm-Vitense, E. 1995a, A\&A, 297, L25

Böhm-Vitense, E. 1995b, AJ, 110, 228

Böhm-Vitense, E., \& Canterna, R. 1974, ApJ, 194, 629

Bois, B., Lanning, H. H., \& Mochnacki, S. W. 1988, AJ, 96, 157

Boss, L. 1908, AJ, 26, 31

Breger, M., Garrido, R., Huang, L., et al. 1989, A\&A, 214, 209

Breger, M., Huang, L., Jiang, S.-Y., et al. 1987, A\&A, 175, 117

Brown, A. 1950, ApJ, 112, 225

de Bruijne, J. H. J. 1999a, MNRAS, 306, 381

de Bruijne, J. H. J. 1999b, MNRAS, 310, 585 (B99b)

de Bruijne, J. H. J., Hoogerwerf, R., \& de Zeeuw, P. T. 2000, ApJL, 544, 65

Brun, A. S., Turck-Chièze, S., \& Morel, P. 1998, ApJ, 506, 913

van Bueren, H. G. 1952, Bull. Astron. Inst. Netherlands, 11, 385

Burkhart, C., \& Coupry, M. F. 1989, A\&A, 220, 197

Burleigh, M. R., Barstow, M. A., \& Holberg, J. B. 1998, MNRAS, 300, 511

Buser, R., \& Kurucz, R. L. 1992, A\&A, 264, 557

Campbell, B. 1984, ApJ, 283, 209

Canuto, V. M., Goldman, I., \& Mazzitelli, I. 1996, ApJ, 473, 550

Canuto, V. M., \& Mazzitelli, I. 1991, ApJ, 370, 295

Canuto, V. M., \& Mazzitelli, I. 1992, ApJ, 389, 724

Castellani, V., Degl'Innocenti, S., \& Prada Moroni, P. G. 2001, MNRAS, 320, 66

Castelli, F. 1999, A\&A, 346, 564

Castelli, F., Gratton, R. G., \& Kurucz, R. L. 1997, A\&A, 318, 841 (erratum A\&A, 324, 432)

Cayrel, R., Cayrel de Strobel, G., \& Campbell, B. 1985, A\&A, 146,249

Chaboyer, B., Demarque, P., \& Pinsonneault, M. H. 1995, ApJ, 441,876

Chabrier, G., \& Baraffe, I. 1997, A\&A, 327, 1039

Chandrasekhar, S. 1961, Hydrodynamic and hydromagnetic stability (Clarendon Press, Oxford)

Chin, C.-W., \& Stothers, R. 1971, ApJ, 163, 555

Clemens, J. C. 1992, ApJ, 391, 773

Code, A. D., Bless, R., Davis, J., \& Brown, R. 1976, ApJ, 203, 417

Collins, G. W., \& Smith, R. C. 1985, MNRAS, 213, 519

Cooke, W. J., \& Eichhorn, H. K. 1997, MNRAS, 288, 319

Cox, J. P., \& Giuli, R. T. 1968, Principles of stellar structure (Gordon and Breach, New York) 
Crawford, D. L. 1975, AJ, 80, 955

Débarbat, S., Eddy, J. A., Eichhorn, H. K., \& Upgren, A. R. 1988, ed. of Mapping the sky, IAU Symp. 133 (Kluwer Acad. Publ., Dordrecht)

Debernardi, Y., Mermilliod, J.-C., Carquillat, J.-M., \& Ginestet, N. 2000, A\&A, 354, 881

Detweiler, H. L., Yoss, K. M., Radick, R. R., \& Becker, S. A. 1984, AJ, 89, 1038

Dobson, A. 1990, PASP, 102, 88

Dravins, D., Lindegren, L., \& Madsen, S. 1999, A\&A, 348, 1040

Dravins, D., Lindegren, L., Madsen, S., \& Holmberg, J. 1997, ESA SP-402, 733

Duflot, M., Figon, P., \& Meyssonnier, N. 1995, A\&AS, 114, 269

ESA 1997, The Hipparcos and Tycho Catalogues, ESA $\mathrm{SP}-1200$

Eggen, O. J. 1983, AJ, 88, 190

Eggen, O. J. 1992, AJ, 104, 1482

Eggen, O. J. 1993, AJ, 106, 642 \& 1885

Faulkner, J. 1967, ApJ, 147, 617

Flower, P. J. 1977, A\&A, 54, 31

Flower, P. J. 1996, ApJ, 469, 355

García Lopéz, R. J., Rebolo, R., Beckman, J. E., \& McKeith, C. D. 1993, A\&A, 273, 482

Gardiner, R. B., Kupka, F., \& Smalley, B. 1999, A\&A, 347, 876

Gatewood, G., Castelaz, M., de Jonge, J. K., et al. 1992, ApJ, 392,710

Girardi, L., Bressan, A., Bertelli, G., \& Chiosi, C. 2000a, A\&AS, 141, 371 (http://pleiadi.pd.astro.it/)

Girardi, L., Mermilliod, J.-C., \& Carraro, G. 2000b, A\&A, 354, 892

Gratton, R. G., Carretta, E., \& Castelli, F. 1996, A\&A, 314, 191

Grevesse, N., \& Noels, A. 1993a, in Association Vaudoise des chercheurs en physique, ed. B. Hauck, S. Plantani, \& D. Raboud

Grevesse, N., \& Noels, A. 1993a, in Origin and evolution of the elements, ed. N. Prantzos, E. Vangioni-Flam, \& M. Casse (Cambridge Univ. Press)

Griffin, R. E. M., \& Holweger, H. 1989, A\&A, 214, 249

Griffin, R. F., \& Gunn, J. E. 1977, AJ, 82, 176

Guinan, E. F., \& Sion, E. M. 1984, AJ, 89, 1252

Gunn, J. E., Griffin, R. F., Griffin, R. E. M., \& Zimmerman, B. A. 1988, AJ, 96, 198

Hamada, T., \& Salpeter, E. E. 1961, ApJ, 134, 683

Hanson, R. B. 1975, AJ, 80, 379

Hardorp, J. 1982, A\&A, 105, 120

Hartmann, L. W., \& Noyes, R. W. 1987, ARA\&A, 25, 271

Hauschildt, P. H., Allard, F., \& Baron, E. 1999, ApJ, 512, 377

Henyey, L. G., Wilets, L., Böhm, K. H., Lelevier, R., \& Levee, R. D. 1959, ApJ, 129, 628

Hodge, P. W., \& Wallerstein, G. 1966, PASP, 78, 411

Hoffleit, E. D., \& Jaschek, C. 1991, The Bright star catalogue, 5th Rev. ed. (Yale Univ. Obs., New Haven)

Høg, E., et al. 2000a, A\&A, 355, L27

Høg, E., et al. 2000b, A\&A, 357, 367

Hoogerwerf, R. 2000, MNRAS, 313, 43

Hoogerwerf, R., \& Aguilar, L. A. 1999, MNRAS, 306, 394

Houdashelt, M. L., Bell, R. A., \& Sweigart, A. V. 2000, AJ, 119,1448

Hubbard, E. N., \& Dearborn, D. S. P. 1980, ApJ, 239, 248

Humason, M. L., \& Zwicky, F. 1947, ApJ, 105, 85
Hünsch, M., Schmitt, J. H. M., \& Voges, W. 1998, A\&AS, 132, 155

Jaschek, C., \& Jaschek, M. 1987, The classification of stars (Cambridge Univ. Press)

Jasniewicz, G. 1984, A\&A, 141, 116

Jensen, K. A., Swank, J. H., Petre, R., et al. 1986, ApJL, 309, 27

Kennelly, E. J., et al. 1996, A\&A, 313, 571

Kippenhahn, R., \& Weigert, A. 1990, Stellar structure and evolution (Springer-Verlag, Berlin)

Kjeldsen, H., \& Frandsen, S. 1991, A\&AS, 87, 119

Koester, D. 1991, in IAU Symp. 145, Evolution of stars, ed. G. Michaud, \& A. V. Tutukov (Kluwer Acad. Publ., Dordrecht), 435

Kraft, R. P., \& Wrubel, M. H. 1965, ApJ, 142, 703

Królikowska, M. 1992, A\&A, 260, 183

Kurucz, R. L. 1991, in Precision photometry, ed. A. G. D. Philip, A. R. Upgren, \& K. A. Janes (Davis Press, Schenectady), 27

Kurucz, R. L. 1995, ATLAS9 CDROM, Castelli et al. 1997

Kurucz, R. L. 2000 [astro-ph/0003069]

Kuzmin, A., Høg, E., Bastian, U., et al. 1999, A\&AS, 136, 491

Lanning, H. H., \& Pesch, P. 1981, ApJ, 244, 280

Lastennet, E., Valls-Gabaud, D., Lejeune, T., \& Oblak, E. 1999, A\&A, 349, 485

Lebreton, Y., Michel, E., Goupil, M. J., Baglin, A., \& Fernandes, J. 1995, in Astronomical and astrophysical objectives of sub-milliarcsecond optical astrometry, ed. E. Høg, \& P. K. Seidelmann (Kluwer Acad. Publ., Dordrecht), 135

Lebreton, Y., Perrin, M.-N., Cayrel, R., Baglin, A., \& Fernandes, J. 1999, A\&A, 350, 587

Lebreton, Y. 2000, ARA\&A, 38, 35

van Leeuwen, F. 1999, A\&A, 341, L71

Lejeune, T., Cuisinier, F., \& Buser, R. 1998, A\&AS, 130, 65

Li, Z. P., \& Michel, E. 1999, A\&A, 344, L41

Lindegren, L. 1989, ESA SP-1111, vol. 3, 311

Lindegren, L., Madsen, S., \& Dravins, D. 2000, A\&A, 356, 1119

Liu, Y. Y., Baglin, A., Auvergne, M., Goupil, M. J., \& Michel, E. 1997, ESA SP-402, 363

Ludwig, H.-G., Freytag, B., \& Steffen, M. 1999, A\&A, 346, 111

Luyten, W. J. 1954, AJ, 59, 224

Luyten, W. J. 1956, AJ, 61, 261

Madsen, S. 1999, in ASP Conf. Ser. 167, Harmonizing cosmic distance scales in a post-Hipparcos era, ed. D. Egret, \& A. Heck, 78

Maeder, A., \& Peytremann, E. 1970, A\&A, 7, 120

Maeder, A., \& Peytremann, E. 1972, A\&A, 21, 279

Maeder, A., \& Meynet, G. 2000, ARA\&A, 38, 143

Makarov, V. V., Odenkirchen, M., \& Urban, S. 2000, A\&A, 358,923

Marsh, M. C., et al. 1997, MNRAS, 286, 369

Mason, B. D., McAlister, H. A., Hartkopf, W. I., \& Bagnuolo, W. G. 1993, AJ, 105, 220

Mayor, M., \& Mazeh, T. 1987, A\&A, 171, 157

Mazzei, P., \& Pigatto, L. 1988, A\&A, 193, 148

McClure, R. D. 1982, ApJ, 254, 606

McWilliam, A. 1990, ApJS, 74, 1075

de Medeiros, J. R., \& Mayor, M. 1999, A\&AS, 139, 433

Mermilliod, J.-C. 1981, A\&AS, 44, 467

Michaud, G. 1986, ApJ, 302, 650

Morel, P. 1997, A\&AS, 124, 597 
Morgan, B. L., Beckmann, G. K., \& Scaddan, R. J. 1982, MNRAS, 198, 817

Murray, C. A., \& Harvey, G. M. 1976, Royal Greenwich Obs. Bull., 182, 15

Narayanan, V. K., \& Gould, A. 1999a, ApJ, 515, 256

Narayanan, V. K., \& Gould, A. 1999b, ApJ, 523, 328

Nelson, B., \& Young, A. 1970, PASP, 82, 699

Nelson, B., \& Young, A. 1972, ApJ, 173, 653

Nelson, G. D. 1980, ApJ, 238, 659

Newberg, H. J., \& Yanny, B. 1998, ApJ, 499, L57

Nordström, B., Andersen, J., \& Andersen, M. I. 1997, A\&A, 322,460

O'Connor, J. 1914, AJ, 28, 175

Pagel, B. E. J., \& Portinari, L. 1998, MNRAS, 298, 747

Pallavicini, R., Micela, G., \& Sciortino, S. 2000, ed. of Stellar clusters and associations, ASP Conf. Ser., vol. 198

Pan, X., Shao, M., \& Colavita, M. M. 1992, in ASP Conf. Ser. 32, Complementary approaches to double and multiple star research, ed. H. A. McAlister, \& W. I. Hartkopf, 502

Patience, J., Ghez, A. M., Reid, I. N., Weinberger, A. J., \& Matthews, K. 1998, AJ, 115, 1972

Pels, G., Oort, J. H., \& Pels-Kluyver, H. A. 1975, A\&A, 43, 423

Pérez Hernández, F., Claret, A., Hernández, M. M., \& Michel, E. 1999, A\&A, 346, 586

Perryman, M. A. C., et al. 1998, A\&A, 331, 81 (P98)

Peterson, D. M. 1991, in ASP Conf. Ser. 13, The formation and evolution of star clusters, ed. K. Janes, 592

Peterson, D. M., Baron, R. L., Dunham, E., \& Mink, D. 1981, AJ, 86, 1090

Peterson, D. M., \& Solensky, R. 1987, ApJ, 315, 286

Peterson, D. M., Stefanik, R. P., \& Latham, D. W. 1993, AJ, 105,2260

Petrie, R. M. 1963, in Basic astronomical data. Stars and stellar systems, vol. III, ed. K. Aa. Strand (Univ. of Chicago Press), 63

Pinsonneault, M. H., Stauffer, J., Soderblom, D. R., King, J. R., \& Hanson, R. B. 1998, ApJ, 504, 170

Rachford, B. L. 1997, ApJ, 486, 994

Rachford, B. L. 1998, ApJ, 505, 255

Rachford, B. L., \& Canterna, R. 2000, AJ, 119, 1296

Reid, N. 1993, MNRAS, 265, 785

Reimers, D. 1975, in Problems in stellar atmospheres and envelopes, ed. B. Baschek, W. H. Kegel, \& G. Traving (Springer-Verlag, New York), 229

Renzini, A., \& Fusi Pecci, F. 1988, ARA\&A, 26, 199

Richer, J., Michaud, G., \& Turcotte, S. 2000, ApJ, 529, 338

Ridgway, S. T., Joyce, R. R., White, N. M., \& Wing, R. F. 1980, ApJ, 235, 126

Robichon, N., Arenou, F., Mermilliod, J.-C., \& Turon, C. 1999, A\&A, 345, 471

Schwan, H. 1991, A\&A, 243, 386

Schwarzschild, M. 1958, Structure and evolution of the stars (Princeton Univ. Press)

Seares, F. H. 1945, ApJ, 102, 366

Shipman, H. L., et al. 1995, AJ, 109, 1220

Simon, T., \& Landsman, W. B. 1997, ApJ, 484, 360

Simon, T., \& Ayres, T. R. 2000, ApJ, 539, 325

Smalley, B., \& Dworetsky, M. M. 1993, A\&A, 271, 515

Smart, W. M. 1939, MNRAS, 100, 60

Smith, G. 1999, A\&A, 350, 859

Smith, G., \& Ruck, M. J. 1997, A\&A, 324, 1091

Söderhjelm, S. 1999, A\&A, 341, 121
Stauffer, J. R., Balachandran, S. C., Krishnamurthi, A., et al. 1997, ApJ, 475, 604

Stauffer, J. R., Giampapa, M. S., Herbst, W., et al. 1991, ApJ, 374,142

Stefanik, R. P., \& Latham, D. W. 1992, in ASP Conf. Ser. 32, Complementary approaches to double and multiple star research, ed. H. A. McAlister, \& W. I. Hartkopf, 173

Stern, R. A., Schmitt, J. H. M. M., Pye, J. P., et al. 1994, ApJ, 427,808

Stern, R. A., Schmitt, J. H. M. M., Rosso, C., et al. 1992, ApJ, 399, L159

Strömgren, B., Olsen, E. H., \& Gustafsson, B. 1982, PASP, 94,5

Swenson, F. J., Faulkner, F., Rogers, F. J., \& Iglesias, C. A. 1994, ApJ, 425, 286

Taylor, B. J. 1980, AJ, 85, 242

Taylor, B. J. 1998, in Fundamental stellar properties, IAU Symp. 189, ed. T. Bedding, A. Booth, \& J. Davis (Kluwer Acad. Publ., Dordrecht), 83

Taylor, B. J. 1999, A\&AS, 134, 523

Terlevich, E. 1987, MNRAS, 224, 193

Tomkin, J., Pan, X., \& McCarthy, J. K. 1995, AJ, 109, 780

Torres, G., Stefanik, R. P., \& Latham, D. W. 1997a, ApJ, 485, 167

Torres, G., Stefanik, R. P., \& Latham, D. W. 1997b, ApJ, 479, 268

Torres, G., Stefanik, R. P., \& Latham, D. W. 1997c, ApJ, 474, 256

Turner, D. G., Garrison, R. F., \& Morris, S. C. 1994, J. Royal Astr. Soc. Canada, 88, 303

Turon, C., et al. 1992, The Hipparcos Input Catalogue, Bull. Inf. Centre Données Stellaires, 41, 9

Udalski, A. 2000, ApJL, 531, 25

Upton, E. K. L. 1970, AJ, 75, 1097

Urban, S. E., Corbin, T. E., \& Wycoff, G. L. 1998a, AJ, 115, 2161

Urban, S. E., Corbin, T. E., Wycoff, G. L., et al. 1998b, AJ, 115,1212

VandenBerg, D. A., \& Bridges, T. A. 1984, ApJ, 278, 679

Varenne, O., \& Monier, R. 1999, A\&A, 351, 247

Wayman, P. A. 1967, PASP, 79, 156

Wayman, P. A., Symms, L. S. T., \& Blackwell, K. C. 1965, Royal Obs. Bull., 98, Ser. E, 33

Weidemann, V., Jordan, S., Iben, I., \& Casertano, S. 1992, AJ, 104,1876

Werner, K., \& Rauch, T. 1997, A\&A, 324, L25

Wesemael, F., van Horn, H. M., Savedoff, M. P., \& Auer, L. H. 1980, ApJS, 43, 159

Wielen, R., Dettbarn, C., Jahreiß, H., Lenhardt, H., \& Schwan, H. 1999, A\&A, 346, 675

Wielen, R., Dettbarn, C., Jahreiß, H., Lenhardt, H., Schwan, H., \& Jährling, R. 2000, in IAU Symp. 200, The formation of binary stars, ed. B. Reipurth, \& H. Zinnecker, 144

Wolff, S. C., Boesgaard, A. M., \& Simon, T. 1986, ApJ, 310, 360

Wood, M. A. 1995, in White dwarfs; proceedings of the 9th European workshop, ed. D. Koester, \& K. Werner (Springer-Verlag, Berlin), 41

Young, A. 1976, ApJ, 205, 182

de Zeeuw, P. T., Hoogerwerf, R., de Bruijne, J. H. J., Brown, A. G. A., \& Blaauw, A. 1999, AJ, 117, 354

Zhao, J. L., \& Chen, L. 1994, A\&A, 287, 68 